The NuSTAR serendipitous survey: the 40-month catalog and the properties of the distant high-energy X-ray source population

Lansbury, G. B.; Stern, D.; Aird, J.; Alexander, D. M.; Fuentes-Yaco, C.; Harrison, F. A.; Treister, E.; Bauer, F. E.; Tomsick, J. A.; Balokovic, M.

Total number of authors:

38

Published in:

Astrophysical Journal

Link to article, DOI:

$10.3847 / 1538-4357 / 836 / 1 / 99$

Publication date:

2017

Document Version

Publisher's PDF, also known as Version of record

Link back to DTU Orbit

Citation (APA):

Lansbury, G. B., Stern, D., Aird, J., Alexander, D. M., Fuentes-Yaco, C., Harrison, F. A., Treister, E., Bauer, F. E., Tomsick, J. A., Balokovic, M., Del Moro, A., Gandhi, P., Ajello, M., Annuar, A., Ballantyne, D. R., Boggs, S. E., Brandt, W. N., Brightman, M., Chen, C-T. J., ... Zhang, W. W. (2017). The NuSTAR serendipitous survey: the 40 -month catalog and the properties of the distant high-energy X-ray source population. Astrophysical Journal, 836(1), [99]. https://doi.org/10.3847/1538-4357/836/1/99

- Users may download and print one copy of any publication from the public portal for the purpose of private study or research.

- You may not further distribute the material or use it for any profit-making activity or commercial gain

- You may freely distribute the URL identifying the publication in the public portal 


\title{
The NuSTAR Serendipitous Survey: The 40-month Catalog and the Properties of the Distant High-energy X-Ray Source Population
}

G. B. Lansbury ${ }^{1,2}$, D. Stern ${ }^{3}$, J. Aird ${ }^{1,2}$, D. M. Alexander ${ }^{1}$, C. Fuentes ${ }^{4}$, F. A. Harrison ${ }^{5}$, E. Treister ${ }^{4,6}$, F. E. Bauer ${ }^{6,7,8}$, J. A. Tomsick ${ }^{9}$, M. Baloković ${ }^{5}$, A. Del Moro ${ }^{1,10}$, P. Gandhi ${ }^{1,11}$, M. Ajello ${ }^{12}$, A. Annuar ${ }^{1}$, D. R. Ballantyne ${ }^{13}$, S. E. Boggs ${ }^{9}$, W. N. Brandt ${ }^{14,15,16}$, M. Brightman ${ }^{5}$, C.-T. J. Chen ${ }^{14}$, F. E. Christensen ${ }^{17}$, F. Civano ${ }^{18,19}$, A. Comastri ${ }^{20}$, W. W. Craig ${ }^{1721}$, K. Forster ${ }^{5}$, B. W. Grefenstette ${ }^{5}$, C. J. Hailey ${ }^{22}$, R. C. Hickox ${ }^{23}$, B. Jiang ${ }^{5}$, H. D. Jun ${ }^{3}$, M. Koss ${ }^{24}$, S. Marchesi ${ }^{12}$, A. D. Melo ${ }^{4}$, J. R. Mullaney ${ }^{25}$, G. Noirot $^{3,26}$, S. Schulze ${ }^{6,7}$, D. J. Walton ${ }^{3,5}$, L. Zappacosta ${ }^{27}$, and W. W. Zhang ${ }^{28}$

${ }^{1}$ Centre for Extragalactic Astronomy, Department of Physics, Durham University, South Road, Durham, DH1 3LE, UK

${ }^{2}$ Institute of Astronomy, University of Cambridge, Madingley Road, Cambridge, CB3 0HA, UK; gbl23@ast.cam.ac.uk 3 Jet Propulsion Laboratory, California Institute of Technology, 4800 Oak Grove Drive, Mail Stop 169-221, Pasadena, CA 91109, USA

${ }^{4}$ Universidad de Concepción, Departamento de Astronomía, Casilla 160-C, Concepción, Chile

${ }^{5}$ Cahill Center for Astrophysics, 1216 East California Boulevard, California Institute of Technology, Pasadena, CA 91125, USA

${ }^{6}$ Instituto de Astrofísica, Facultad de Física, Pontificia Universidad Católica de Chile, 306, Santiago 22, Chile

${ }^{7}$ Millennium Institute of Astrophysics, Vicuña Mackenna 4860, 7820436 Macul, Santiago, Chile

${ }^{8}$ Space Science Institute, 4750 Walnut Street, Suite 205, Boulder, CO 80301, USA

${ }_{10}^{9}$ Space Sciences Laboratory, 7 Gauss Way, University of California, Berkeley, CA 94720-7450, USA

${ }_{10}^{10}$ Max-Planck-Institut für Extraterrestrische Physik (MPE), Postfach 1312, D-85741, Garching, Germany

${ }^{11}$ School of Physics and Astronomy, University of Southampton, Highfield, Southampton SO17 1BJ, UK

${ }^{12}$ Department of Physics and Astronomy, Clemson University, Clemson, SC 29634-0978, USA

${ }_{14}^{13}$ Center for Relativistic Astrophysics, School of Physics, Georgia Institute of Technology, Atlanta, GA 30332, USA

${ }^{14}$ Department of Astronomy and Astrophysics, The Pennsylvania State University, University Park, PA 16802, USA

${ }^{15}$ Institute for Gravitation and the Cosmos, The Pennsylvania State University, University Park, PA 16802, USA

${ }^{17}$ DTU Space-National Space Institute, Technical University of Denmark, Elektrovej 327, DK-2800 Lyngby, Denmark

${ }^{18}$ Yale Center for Astronomy and Astrophysics, Physics Department, Yale University, New Haven, CT 06520, USA

${ }^{19}$ Harvard-Smithsonian Center for Astrophysics, 60 Garden Street, Cambridge, MA 02138, USA

${ }^{20}$ INAF Osservatorio Astronomico di Bologna, via Ranzani 1, I-40127 Bologna, Italy

${ }^{21}$ Lawrence Livermore National Laboratory, Livermore, CA 94550, USA

${ }^{22}$ Columbia Astrophysics Laboratory, 550 W. 120th Street, Columbia University, NY 10027, USA

23 Department of Physics and Astronomy, Dartmouth College, 6127 Wilder Laboratory, Hanover, NH 03755, USA

${ }^{24}$ Institute for Astronomy, Department of Physics, ETH Zurich, Wolfgang-Pauli-Strasse 27, CH-8093 Zurich, Switzerland

${ }_{26}$ Department of Physics and Astronomy, The University of Sheffield, Hounsfield Road, Sheffield S3 7RH, UK

${ }^{26}$ Université Paris-Diderot Paris VII, Université de Paris Sorbonne Cité (PSC), F-75205 Paris Cedex 13, France

${ }^{27}$ INAF Osservatorio Astronomico di Roma, via Frascati 33, 00040 Monte Porzio Catone (RM), Italy

${ }^{28}$ NASA Goddard Space Flight Center, Greenbelt, MD 20771, USA

Received 2016 September 2; revised 2016 November 18; accepted 2016 December 7; published 2017 February 10

\begin{abstract}
We present the first full catalog and science results for the Nuclear Spectroscopic Telescope Array (NuSTAR) serendipitous survey. The catalog incorporates data taken during the first 40 months of NuSTAR operation, which provide $\approx 20 \mathrm{Ms}$ of effective exposure time over 331 fields, with an areal coverage of $13 \mathrm{deg}^{2}$, and 497 sources detected in total over the $3-24 \mathrm{keV}$ energy range. There are 276 sources with spectroscopic redshifts and classifications, largely resulting from our extensive campaign of ground-based spectroscopic follow-up. We characterize the overall sample in terms of the X-ray, optical, and infrared source properties. The sample is primarily composed of active galactic nuclei (AGNs), detected over a large range in redshift from $z=0.002$ to 3.4 (median of $\langle z\rangle=0.56$ ), but also includes 16 spectroscopically confirmed Galactic sources. There is a large range in $\mathrm{X}$-ray flux, from $\log \left(f_{3-24 \mathrm{keV}} / \mathrm{erg} \mathrm{s}^{-1} \mathrm{~cm}^{-2}\right) \approx-14$ to -11 , and in rest-frame $10-40 \mathrm{keV}$ luminosity, from $\log \left(L_{10-40 \mathrm{keV}} / \mathrm{erg} \mathrm{s}^{-1}\right) \approx 39$ to 46 , with a median of 44.1 . Approximately $79 \%$ of the NuSTAR sources have lower-energy $(<10 \mathrm{keV}) \mathrm{X}$-ray counterparts from XMM-Newton, Chandra, and Swift XRT. The mid-infrared (MIR) analysis, using WISE all-sky survey data, shows that MIR AGN color selections miss a large fraction of the NuSTAR-selected AGN population, from $\approx 15 \%$ at the highest luminosities $\left(L_{\mathrm{X}}>10^{44} \mathrm{erg} \mathrm{s}^{-1}\right)$ to $\approx 80 \%$ at the lowest luminosities $\left(L_{\mathrm{X}}<10^{43} \mathrm{erg} \mathrm{s}^{-1}\right)$. Our optical spectroscopic analysis finds that the observed fraction of optically obscured AGNs (i.e., the type 2 fraction) is $F_{\text {Type } 2}=53_{-15}^{+14} \%$, for a well-defined subset of the $8-24 \mathrm{keV}$ selected sample. This is higher, albeit at a low significance level, than the type 2 fraction measured for redshift- and luminosity-matched AGNs selected by $<10 \mathrm{keV}$ X-ray missions.
\end{abstract}

Key words: catalogs - galaxies: active - galaxies: nuclei - quasars: general - surveys - X-rays: general

Supporting material: figure sets, machine-readable tables

\section{Introduction}

Since the late 1970s, which saw the advent of focusing X-ray observatories in space (e.g., Giacconi et al. 1979), $\mathrm{X}$-ray surveys have provided fundamental advances in our understanding of growing supermassive black holes (e.g., Fabian \& Barcons 1992; Brandt \& Hasinger 2005; Alexander \& Hickox 2012; Brandt \& Alexander 2015). X-rays provide the most direct and efficient means of identifying active galactic 
nuclei (AGNs; the sites of rapid mass accretion onto supermassive black holes), since the effects of both line-of-sight absorption and dilution by host-galaxy light are comparatively low at X-ray energies. The X-ray surveys over the past few decades have ranged from wide-area all-sky surveys to deep pencil-beam surveys, allowing the evolution of AGN obscuration and the X-ray luminosity function to be measured for a wide range in luminosity and redshift (up to $z \approx 5$; e.g., see Brandt \& Alexander 2015 for a review). The deepest surveys with Chandra and XMM-Newton have directly resolved the majority $(\approx 70 \%-90 \%)$ of the $\lesssim 8 \mathrm{keV}$ cosmic X-ray background (CXB) into individual objects (e.g., Worsley et al. 2005; Hickox \& Markevitch 2006; Xue et al. 2012).

Until very recently, the most sensitive X-ray surveys (e.g., with Chandra and XMM-Newton) have been limited to photon energies of $<10 \mathrm{keV}$ and are therefore biased against the identification of heavily obscured AGNs (for which the line-ofsight column density exceeds $N_{\mathrm{H}} \sim$ a few $\times 10^{23} \mathrm{~cm}^{-2}$ ). This bias is especially strong at $z \lesssim 1$, but becomes less so for higher redshifts, where the spectral features of absorption, and the penetrating higher-energy X-rays, are shifted into the observed-frame X-ray energy window. The result is a complicated AGN selection function, which is challenging to correct for without full knowledge of the prevalence of highly absorbed systems. These photon energies are also low compared to the peak of the CXB (at $\approx 20-30 \mathrm{keV}$ ), meaning that spectral extrapolations are required to characterize the AGN population responsible for the CXB peak. High-energy $(>10 \mathrm{keV}) \mathrm{X}$-ray surveys with nonfocusing X-ray observatories (e.g., Swift BAT and INTEGRAL) have directly resolved $\approx 1 \%-2 \%$ of the CXB peak into individual AGNs (Krivonos et al. 2007; Ajello et al. 2008; Bottacini et al. 2012). These surveys have been successful in characterizing the local highenergy-emitting AGN population (e.g., Tueller et al. 2008; Burlon et al. 2011; Vasudevan et al. 2013; Ricci et al. 2015), but, being largely confined to $z \lesssim 0.1$, there is limited scope for evolutionary studies.

A great breakthrough in studying the high-energy X-rayemitting population is the Nuclear Spectroscopic Telescope Array (NuSTAR; Harrison et al. 2013), the first orbiting observatory with the ability to focus X-ray light at energies $>10 \mathrm{keV}$, resulting in a two-order-of-magnitude increase in sensitivity over previous nonfocusing missions. This has opened up the possibility to study large, cleanly selected samples of high-energy-emitting AGNs in the distant universe for the first time. The NUSTAR extragalactic survey program has provided the first measurements of the $>10 \mathrm{keV}$ AGN luminosity functions at $z>0.1$ (Aird et al. 2015a) and has directly resolved a large fraction $(35 \pm 5 \%)$ of the CXB at 8-24 keV (Harrison et al. 2016). In addition, both the survey program and targeted NUSTAR campaigns have demonstrated the importance of high-energy coverage for accurately constraining the intrinsic properties of distant AGNs (e.g., Del Moro et al. 2014; Lansbury et al. 2014, 2015; Luo et al. 2014; Civano et al. 2015; LaMassa et al. 2016), especially in the case of the most highly absorbed Compton-thick (CT) systems (where $N_{\mathrm{H}}>1.5 \times 10^{24} \mathrm{~cm}^{-2}$ ).

The NUSTAR extragalactic survey is the largest scientific program, in terms of time investment, undertaken with NUSTAR and is one of the highest priorities of the mission. There are two main "blind survey" components. First, deep blank-field NUSTAR surveys have been performed in the following well- studied fields: the Extended Chandra Deep Field South (ECDFS; Lehmer et al. 2005), for which the total areal coverage with $N u S T A R$ is $\approx 0.33 \mathrm{deg}^{2}$ (Mullaney et al. 2015, hereafter M15); the Cosmic Evolution Survey field (COSMOS; Scoville et al. 2007), which has $\approx 1.7 \mathrm{deg}^{2}$ of NuSTAR coverage (Civano et al. 2015, hereafter C15); the Extended Groth Strip (EGS; Groth et al. 1994), with $\approx 0.25 \mathrm{deg}^{2}$ of coverage (J. Aird et al. 2017, in preparation); the northern component of the Great Observatories Origins Deep Survey North (GOODS-N; Dickinson et al. 2003), with $\approx 0.07 \mathrm{deg}^{2}$ of coverage (A. Del Moro et al. 2017, in preparation); and the Ultra Deep Survey field (UDS; Lawrence et al. 2007), with $\approx 0.6 \mathrm{deg}^{2}$ of coverage (A. Masini et al. 2017, in preparation). Second, a wide-area "serendipitous survey" has been performed by searching the majority of NUSTAR pointings for chance background sources. An initial look at 10 serendipitous survey sources was presented in Alexander et al. (2013). Serendipitous surveys represent an efficient and economical way to sample wide sky areas, and provide substantial data sets with which to examine the X-ray-emitting population and search for extreme sources. They have been undertaken with many X-ray missions over the past few decades (e.g., Gioia et al. 1990; Comastri et al. 2001; Fiore et al. 2001; Harrison et al. 2003; Nandra et al. 2003; Gandhi et al. 2004; Kim et al. 2004; Ueda et al. 2005; Watson et al. 2009; Evans et al. 2010, 2014).

In this paper, we describe the NUSTAR serendipitous survey and present the first catalog, compiled from data that span the first 40 months of NUSTAR operation. The serendipitous survey is a powerful component of the NUSTAR survey program, with the largest overall sample size, the largest areal coverage $\left(\approx 13 \mathrm{deg}^{2}\right)$, and regions with comparable sensitivity to the other NUSTAR surveys in well-studied fields. Section 2 details the NUSTAR observations, data reduction, source detection, and photometry. We match to counterparts at lower X-ray energies (from Chandra, XMM-Newton, and Swift XRT; Section 3.1) and at optical and infrared (IR) wavelengths (Section 3.2). We have undertaken an extensive campaign of ground-based spectroscopic follow-up, crucial for obtaining source redshifts and classifications, which is described in Section 3.3. Our results for the X-ray, optical, and IR properties of the overall sample are presented in Sections 4.1-4.3, respectively. We summarize the main results in Section 5. All uncertainties and limits are quoted at the $90 \%$ confidence level, unless otherwise stated. We assume the flat $\Lambda \mathrm{CDM}$ cosmology from WMAP7 (Komatsu et al. 2011).

\section{The NuSTAR Data}

The NUSTAR observatory (launched in 2012 June; Harrison et al. 2013) is composed of two independent telescopes (A and B), identical in design, the focal plane modules (FPMs) of which are hereafter referred to as FPMA and FPMB. The modules have fields of view (FOVs) of $\approx 12^{\prime} \times 12^{\prime}$, which overlap in sky coverage. The observatory is sensitive between 3 and $78 \mathrm{keV}$. The main energy band that we focus on here is the $3-24 \mathrm{keV}$ band; this is the most useful band for the relatively faint sources detected in the NUSTAR extragalactic surveys, since the combination of instrumental background and a decrease in effective area with increasing energy means that source photons are unlikely to be detected at higher energies (except for the brightest sources). NUSTAR provides an improvement of over an order of magnitude in angular 
Table 1

Details of the Individual NuSTAR Observations That Make Up the Serendipitous Survey

\begin{tabular}{|c|c|c|c|c|c|c|c|c|c|c|}
\hline $\begin{array}{l}\text { Field ID } \\
\text { (1) }\end{array}$ & $\begin{array}{l}\text { Science Target } \\
\text { (2) }\end{array}$ & $\begin{array}{c}N_{\text {obs }} \\
(3)\end{array}$ & $\begin{array}{l}\text { Obs. ID } \\
\text { (4) }\end{array}$ & $\begin{array}{l}\text { Obs. Date } \\
\text { (5) }\end{array}$ & $\begin{array}{l}\text { R.A. (deg) } \\
\text { (6) }\end{array}$ & $\begin{array}{l}\text { Decl. (deg) } \\
\text { (7) }\end{array}$ & $\begin{array}{c}t_{\exp }(\mathrm{ks}) \\
\quad(8)\end{array}$ & $\begin{array}{l}N_{\text {serendips }} \\
\text { (9) }\end{array}$ & $\begin{array}{l}\text { A15 } \\
(10)\end{array}$ & $\begin{array}{l}\mathrm{H} 16 \\
(11)\end{array}$ \\
\hline 1 & 2MASX J05081967+1721483 & 1 & 60006011002 & 2012 Jul 23 & 77.08 & 17.36 & 16.6 & 0 & 0 & 0 \\
\hline 2 & Bkgd BII -11.2 & 1 & 10060003001 & 2012 Jul 24 & 71.11 & 28.38 & 8.9 & 0 & 0 & 0 \\
\hline 3 & 2MASX J04234080+0408017 & 2 & $\ldots$ & $\ldots$ & $\ldots$ & $\ldots$ & 12.3 & 2 & 1 & 1 \\
\hline $3 \mathrm{a}$ & $\cdots$ & $\cdots$ & 60006005002 & 2012 Jul 25 & 65.92 & 4.13 & 6.4 & $\cdots$ & $\cdots$ & $\cdots$ \\
\hline $3 \mathrm{~b}$ & $\cdots$ & $\cdots$ & 60006005003 & 2012 Jul 25 & 65.92 & 4.13 & 5.9 & $\cdots$ & $\cdots$ & $\cdots$ \\
\hline 4 & IC $4329 \mathrm{~A}$ & 1 & 60001045002 & 2012 Aug 12 & 207.33 & -30.31 & 177.3 & 2 & 0 & 1 \\
\hline 5 & Mrk 231 & 2 & $\ldots$ & $\ldots$ & $\ldots$ & $\ldots$ & 74.9 & 4 & 1 & 1 \\
\hline $5 \mathrm{a}$ & $\cdots$ & $\cdots$ & 60002025002 & 2012 Aug 26 & 194.06 & 56.87 & 44.3 & $\cdots$ & $\cdots$ & $\cdots$ \\
\hline $5 b$ & $\ldots$ & $\ldots$ & 60002025004 & 2013 May 09 & 194.06 & 56.87 & 30.6 & $\ldots$ & $\ldots$ & $\ldots$ \\
\hline 6 & NGC 7582 & 2 & $\ldots$ & $\ldots$ & $\ldots$ & $\ldots$ & 33.4 & 2 & 0 & 1 \\
\hline $6 \mathrm{a}$ & $\cdots$ & $\cdots$ & 60061318002 & 2012 Aug 31 & 349.60 & -42.37 & 17.7 & $\cdots$ & $\cdots$ & $\cdots$ \\
\hline $6 \mathrm{~b}$ & $\cdots$ & $\cdots$ & 60061318004 & 2012 Sep 14 & 349.60 & -42.37 & 15.7 & $\cdots$ & $\cdots$ & $\cdots$ \\
\hline 7 & $\mathrm{AE}$ Aqr & 4 & $\ldots$ & $\ldots$ & $\ldots$ & $\ldots$ & 134.2 & 2 & 1 & 1 \\
\hline $7 \mathrm{a}$ & $\cdots$ & $\cdots$ & 30001120002 & 2012 Sep 04 & 310.04 & -0.87 & 7.2 & $\cdots$ & $\cdots$ & $\cdots$ \\
\hline $7 \mathrm{~b}$ & $\ldots$ & $\cdots$ & 30001120003 & 2012 Sep 05 & 310.04 & -0.87 & 40.5 & $\cdots$ & $\cdots$ & $\ldots$ \\
\hline $7 \mathrm{c}$ & $\cdots$ & $\cdots$ & 30001120004 & 2012 Sep 05 & 310.04 & -0.87 & 76.6 & $\cdots$ & $\cdots$ & $\ldots$ \\
\hline $7 \mathrm{~d}$ & $\cdots$ & $\cdots$ & 30001120005 & 2012 Sep 07 & 310.04 & -0.87 & 9.8 & $\cdots$ & $\cdots$ & $\cdots$ \\
\hline 8 & NGC 612 & 1 & 60061014002 & 2012 Sep 14 & 23.49 & -36.49 & 17.9 & 0 & 0 & 1 \\
\hline 9 & $3 C 382$ & 1 & 60061286002 & 2012 Sep 18 & 278.76 & 32.70 & 18.0 & 1 & 0 & 0 \\
\hline 10 & PBC J1630.5+3924 & 1 & 60061271002 & 2012 Sep 19 & 247.64 & 39.38 & 17.1 & 1 & 1 & 1 \\
\hline
\end{tabular}

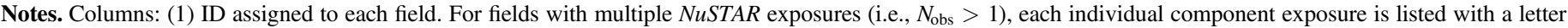

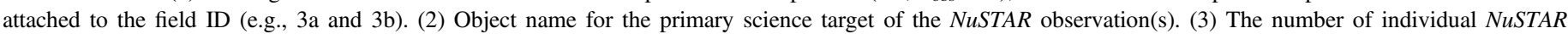

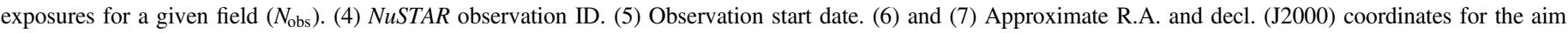

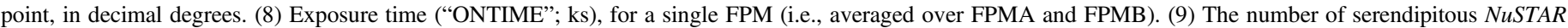

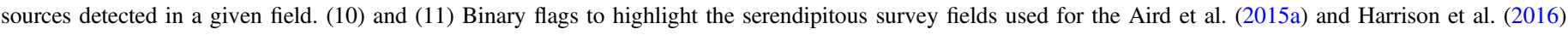
studies, respectively. This table shows the first 10 (out of 331) fields only.

(This table is available in its entirety in machine-readable form.)

resolution compared to previous-generation hard X-ray observatories: the point-spread function (PSF) has an FWHM of $18^{\prime \prime}$ and a half-power diameter of $58^{\prime \prime}$ and is relatively constant across the FOV. The astrometric accuracy of NuSTAR is $8^{\prime \prime}$ for the brightest targets (90\% confidence; Harrison et al. 2013). This worsens with decreasing photon counts, reaching a positional accuracy of $\approx 20^{\prime \prime}$ for the faintest sources (as we demonstrate in Section 3.1).

Here we describe the observations, data reduction, and data analysis procedures used for the NuSTAR serendipitous survey. Section 2.1 describes the NUSTAR observations that have been incorporated as part of the survey. Section 2.2 details the data reduction procedures used to generate the NUSTAR science data. Section 2.3 provides details of the source detection approach. Section 2.4 outlines the photometric measurements for source counts, band ratios, fluxes, and luminosities. Section 2.5 describes the final source catalog.

\subsection{The Serendipitous Survey Observations}

The serendipitous survey is the largest-area blind survey undertaken with NUSTAR. The survey is achieved by searching the background regions of almost every non-survey $N u S T A R$ pointing for background sources unassociated with the original science target. The survey approach is well suited to NuSTAR since there are generally large regions of uncontaminated background. We exclude from the survey NUSTAR fields with bright science targets, identified as fields with $>10^{6}$ counts within $120^{\prime \prime}$ of the on-axis position. We also exclude the dedicated extragalactic (COSMOS, ECDFS, EGS, GOODS-N, UDS) and Galactic survey fields (the Galactic center survey;
Mori et al. 2015; Hong et al. 2016; and the Norma Arm survey; F. Fornasini et al. 2017, in preparation).

Over the period from 2012 July to 2015 November, which is the focus of the current study, there are 510 individual $N u S T A R$ exposures that have been incorporated into the serendipitous survey. These exposures were performed over 331 unique fields (i.e., 331 individual sky regions, each with contiguous coverage composed of one or more NUSTAR exposures), yielding a total sky area coverage of $13 \mathrm{deg}^{2}$. Table 1 lists the fields chronologically ${ }^{29}$ and provides the following details for each field: the name of the primary $N u S T A R$ science target, the number of $N U S T A R$ exposures, the individual NuSTAR observation ID(s), the observation date(s), the pointing coordinates, the exposure time(s), the number of serendipitous sources detected, and flags to indicate the NUSTAR fields that were used in the Aird et al. (2015a) and Harrison et al. (2016) studies. Figure 1 shows an all-sky map of the serendipitous survey fields. The fields have a cumulative exposure time of 20.4 Ms. For comparison, the NuSTAR surveys of COSMOS and ECDFS have cumulative exposure times of 3.1 and $1.5 \mathrm{Ms}$ (C15 and M15, respectively). The serendipitous survey fields cover a wide range in individual exposure times (from $\sim 10 \mathrm{ks}$ to $1 \mathrm{Ms}$ ) and have a median exposure of $28 \mathrm{ks}$ (these values correspond to a single $N u S T A R$ FPM). For $76 \%$ of the fields there is a single $N u S T A R$ exposure, and for the remainder there are multiple (from 2 to 15 ) exposures that are combined together for the science analyses (see Section 2.2).

\footnotetext{
${ }^{29}$ In Table 1 we show the first 10 fields as an example. The full table, which includes all 331 fields, is available online.
} 


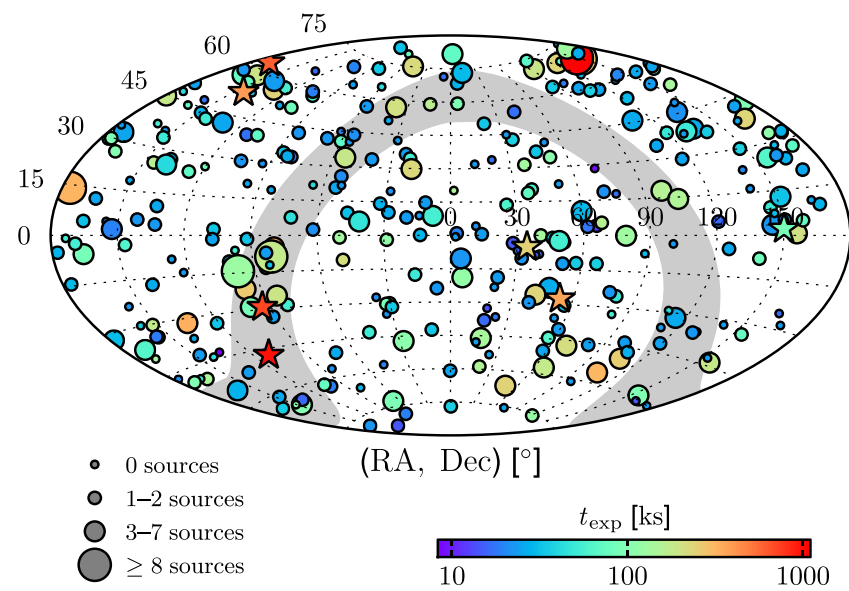

Figure 1. Aitoff projection showing the distribution of NuSTAR serendipitous survey fields on the sky, in equatorial coordinates (R.A., decl.). The circle sizes correspond to the number of sources detected in a given field, and the colors correspond to the cumulative exposure time (per FPM) for a given field. The locations of the dedicated NUSTAR surveys in well-studied fields (COSMOS, ECDFS, EGS, GOODS-N, UDS, the Galactic center, and the Norma Arm), which are not included in the serendipitous survey, are marked with stars (with the colors representing the maximum unvignetted exposures). Also excluded from the serendipitous survey are $N U S T A R$ fields containing bright targets (not shown in this figure; see Section 2.1). The gray area highlights the region $\pm 10^{\circ}$ of the Galactic plane.

An important contributor of fields to the NUSTAR serendipitous survey is the NUSTAR "snapshot survey" (Baloković et al. 2014; M. Baloković et al. 2017, in preparation), a dedicated NuSTAR program targetting Swift-BAT-selected AGNs (the Swift BAT AGNs themselves are not included in the serendipitous survey, only the background regions of the NUSTAR observations). For this work we include 154 snapshot survey fields observed during the first 40 months of NuSTAR operation. These yield $21 \%$ of the total serendipitous survey source detections and make up a large fraction of the survey area (accounting for $47 \%$ of the fields incorporated, in total).

\subsection{Data Processing}

For data reduction, we use HEASoft v. 6.15, the NuSTAR Data Analysis Software (NuSTARDAS) v. 1.3.0, ${ }^{30}$ and CIAO v. 4.8. For each of the 510 obsIDs incorporated in the survey, the raw, unfiltered event files for FPMA and FPMB were processed using the NUPIPELINE program to yield calibrated, cleaned event files. For source detection and photometry (see Sections 2.3 and 2.4), we adopt the observed-frame energy bands that have been utilized for the NUSTAR extragalactic survey program in general and other recent NuSTAR studies: 3-8 keV, 3-24 keV, and 8-24 keV (hereafter referred to as the soft, full, and hard bands, respectively; e.g., Alexander et al. 2013; Luo et al. 2014; Aird et al. 2015a; Lansbury et al. 2015; Harrison et al. 2016). To produce individual energy-band images from the NUSTAR event lists, we used the CIAO program DMCOPY (Fruscione et al. 2006).

To produce exposure maps, which account for the natural dither of the observatory and regions of lower sensitivity (e.g., chip gaps), we follow the procedure outlined in detail in Section 2.2.3 of M15. Vignetting in the optics results in a decrease in the effective exposure with increasing distance from the optical axis. We produce both vignetting-corrected

\footnotetext{
${ }^{30}$ http://heasarc.gsfc.nasa.gov/docs/nustar/analysis
}

and non-vignetting-corrected exposure maps. The former allow us to determine the effective exposure at source positions within the FOV and correctly determine count rates, while the latter are more appropriate for the scaling of background counts since the NUSTAR aperture background component dominates the background photon counts at $\lesssim 30 \mathrm{keV}$ (e.g., Wik et al. 2014).

In order to increase sensitivity, we perform source detection (see Section 2.3) and photometry (see Section 2.4) on the coadded FPMA+FPMB (hereafter "A+B") data, produced by combining the FPMA and FPMB science data with the HEASoft package XIMAGE. For fields with multiple obsIDs, we use XIMAGE to combine the data from individual observations, such that each field has a single mosaic on which source detection and photometry are performed.

\subsection{Source Detection}

In general, the source detection procedure follows that adopted in the dedicated blank-field surveys (e.g., see C15 and M15). A significant difference with the serendipitous survey, compared to the blank-field surveys, is the existence of a science target at the FOV aim point. We account for the background contribution from such science targets by incorporating them in the background map generation, as described below. We also take two steps to exclude sources associated with the science target: (1) in cases where the target has an extended counterpart in the optical or IR bands (e.g., a lowredshift galaxy or galaxy cluster), we mask out custom-made regions that are conservatively defined to be larger than the extent of the counterpart in the optical imaging coverage (from the Sloan Digital Sky Survey [SDSS] or Digitized Sky Survey [DSS]), accounting for spatial smearing of emission due to the NuSTAR PSF; (2) for all point-source detections with spectroscopic identifications, we assign an "associated" flag to those that have a velocity offset from the science target $[\Delta(c z)]$ smaller than $5 \%$ of the total science target velocity.

Here we summarize the source detection procedure, which is applied separately for each of the individual NuSTAR energy bands (soft, full, and hard) before the individual band source lists are merged to form the final catalog. For every pixel position across the NUSTAR image, a "false probability" is calculated to quantify the chance that the counts measured in a source detection aperture around that position are solely due to a background fluctuation. In this calculation we adopt a circular source detection aperture of radius $20^{\prime \prime}$, which is justified by the tight core of the NuSTAR PSF (FWHM = 18") and was also adopted for the dedicated blank-field surveys (e.g., C15; M15). To measure the background level at each pixel position, background counts are first measured from the NUSTAR image using an annular aperture of inner radius $45^{\prime \prime}$ and outer radius $90^{\prime \prime}$, centered on that position. These background counts are then rescaled to the $20^{\prime \prime}$ source detection aperture according to the ratio of effective areas (as determined from non-vignettingcorrected exposure maps). This approach allows the local background to be sampled without significant contamination from the serendipitous source counts (since the background annulus has a relatively large inner radius). The background measurement also accounts for any contaminating photons from the aim-point science target that, due to the broad wings of the NUSTAR PSF, can contribute to the background (if the science target is comparatively bright and offset by $\lesssim 200^{\prime \prime}$ from the serendipitous source position). The Poisson false 
probability $\left(P_{\text {False }}\right)$ is assessed at each pixel, using the source and scaled background counts (e.g., Lehmer et al. 2005; Nandra et al. 2005; Laird et al. 2009), to yield a $P_{\text {False }}$ map. From this map we exclude areas within $30^{\prime \prime}$ of the lowexposure $(<10 \%$ of the maximum exposure) peripheral regions close to the FOV edge, where there is a steep drop-off in exposure and the background is poorly characterized.

We then perform source detection on the $P_{\text {False }}$ map to identify sources. For a full, detailed description of this source detection procedure we refer the reader to Section 2.3 of M15. In brief, the SExtractor algorithm (Bertin \& Arnouts 1996) is used to identify regions of each $P_{\text {False }}$ map that fall below a threshold of $\log \left(P_{\text {False }}\right)<-6$ (the approximate average of the thresholds adopted for the NUSTAR-COSMOS and NuSTARECDFS surveys; C15; M15), producing source lists for each individual energy band. The coordinates for each detected source are measured at the local minimum in $P_{\text {False }}$. Finally, we merge the sources detected in the different energy bands to yield a final source list. To achieve this band merging, the softband (3-8 keV) and hard-band ( $8-24 \mathrm{keV})$ detected sources are matched to the full-band $(3-24 \mathrm{keV})$ source list using a matching radius of $35^{\prime \prime}$. The adopted NUSTAR source coordinates correspond to the position of the source in the full band, if there is a detection in this band. Otherwise, the coordinates correspond to the soft band, if there is a detection in this band, or the hard band if there is no full- or soft-band detection. The analyses described below (e.g., photometry and multiwavelength counterpart matching) are performed using these adopted source coordinates. After the above source detection has been performed, we exclude any sources within $90^{\prime \prime}$ of the central science target position (for comparison, the half-power diameter of the NUSTAR PSF is $58^{\prime \prime}$ ).

To determine the overall sky coverage of the survey as a function of flux sensitivity, we sum the sensitivity curves for the 331 individual fields. For each field the sensitivity curve is determined by calculating, for every point in the NUSTAR image (excluding the low-exposure peripheral regions), the flux limit corresponding to $\log \left(P_{\text {False }}\right)=-6$ (the detection threshold), given the background and exposure maps described above and the count-rate-to-flux conversion factors listed in Section 2.4. Figure 2 shows the total, summed sensitivity curves for the serendipitous survey, for the three main energy bands. Figure 3 shows the logarithmic version, compared to the other components of the NUSTAR extragalactic survey program. The serendipitous survey has the largest solid angle coverage for most fluxes and a similar areal coverage to the deepest blankfield survey (the NUSTAR-EGS survey) at the lowest flux limits. In both Figures 2 and 3 we also show the area curves for the subset of the serendipitous survey that lies outside of the Galactic plane $\left(|b|>10^{\circ}\right)$ and is thus relatively free of Galactic sources. We note that the recent works of Aird et al. (2015a) and Harrison et al. (2016), which presented the luminosity functions and source number counts for the NUSTAR extragalactic survey program, only incorporated serendipitous survey fields at $|b|>20^{\circ}$ (and at decl. $>-5^{\circ}$ for Aird et al. 2015a).

\subsection{Photometry}

For each source detected using the above procedure we measure the net counts, count rates, and fluxes, and for sources with spectroscopic redshifts we calculate rest-frame luminosities. For the aperture photometry, we adopt a circular aperture of $30^{\prime \prime}$ radius to measure the gross (i.e., source plus

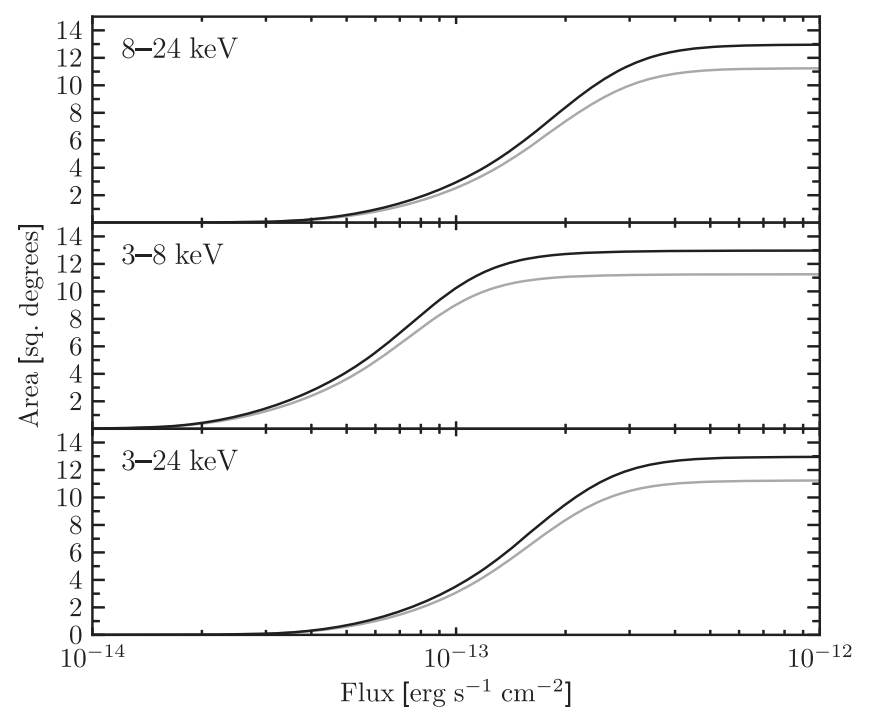

Figure 2. Sky coverage (solid angle) of the NUSTAR serendipitous survey as a function of (aperture-corrected) flux sensitivity, for the three main energy bands. The black line shows the area curve for the full survey, and the gray line shows that for the survey regions outside the Galactic plane $\left(|b|>10^{\circ}\right)$.

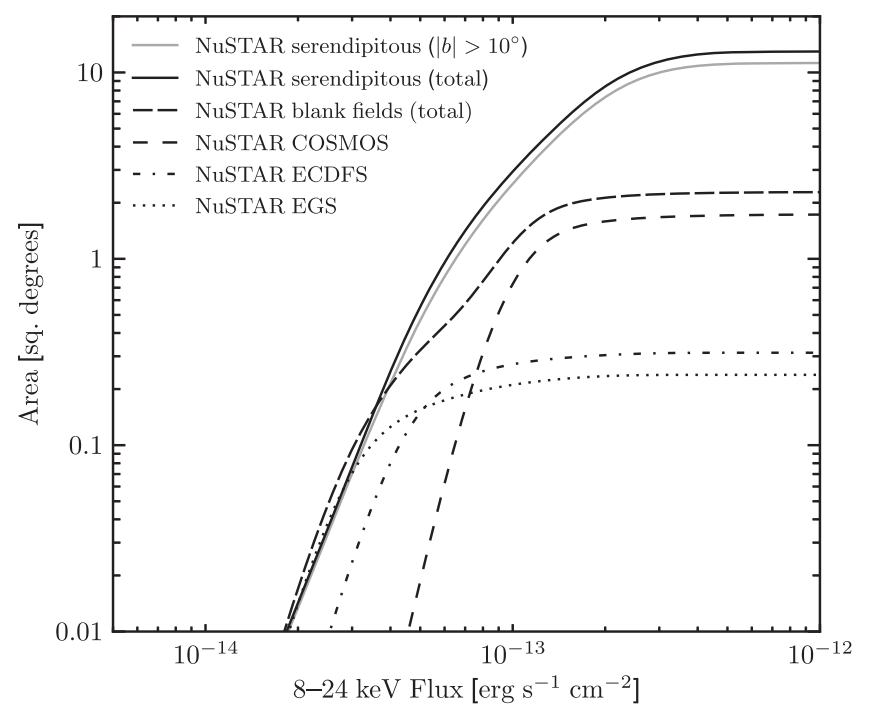

Figure 3. Sky coverage (solid angle) of the NUSTAR serendipitous survey as a function of flux sensitivity, for the hard (8-24 keV) energy band, at which NUSTAR is uniquely sensitive, i.e., the sky coverage for which sources above a given hard-band flux will be detected in the hard band. The black and gray solid lines show the area curves for the overall and the $|b|>10^{\circ}$ serendipitous survey, respectively. We compare with the other completed components of the $N U S T A R$ extragalactic survey program, which include the following dedicated blank-field surveys: NUSTAR-COSMOS (dashed line; C15), NuSTAR-ECDFS (dot-dashed line; M15), and NuSTAR-EGS (dotted line; J. Aird et al. 2017, in preparation). The total area for these blank-field surveys (which are not included as part of the serendipitous survey) is shown as a long-dashed line.

background) counts $(S)$. The scaled background counts $\left(B_{\text {src }}\right)$ are determined using the same procedure as for the source detection (Section 2.3) and are subtracted from $S$ to obtain the net source counts $\left(S_{\text {net }}\right)$. The errors on $S_{\text {net }}$ are computed as $1+\sqrt{S+0.75}$ (84\% confidence level; e.g., Gehrels 1986). For sources undetected in a given band, upper limits for $S_{\text {net }}$ are calculated using the Bayesian approach outlined in Kraft et al. (1991). To determine the net count rate, we divide $S_{\text {net }}$ by the exposure time drawn from the vignetting-corrected exposure map (mean value within the $30^{\prime \prime}$ aperture). 
Table 2

Source Statistics for the NuSTAR Serendipitous Survey

\begin{tabular}{llr}
\hline \hline Band & $N$ & $N_{z}$ \\
$(1)$ & $(2)$ & $(3)$ \\
\hline Any band & 497 & 276 \\
$\mathrm{~F}+\mathrm{S}+\mathrm{H}$ & $104(21 \%)$ & 77 \\
$\mathrm{~F}+\mathrm{S}$ & $116(23 \%)$ & 82 \\
$\mathrm{~F}+\mathrm{H}$ & $35(7 \%)$ & 21 \\
$\mathrm{~S}+\mathrm{H}$ & $0(0 \%)$ & 0 \\
$\mathrm{~F}$ & $165(33 \%)$ & 77 \\
$\mathrm{~S}$ & $53(11 \%)$ & 16 \\
$\mathrm{H}$ & $24(5 \%)$ & 3 \\
\hline
\end{tabular}

Notes. (1) F, S, and $\mathrm{H}$ refer to sources detected in the full (3-24 keV), soft (3-8 keV), and hard (8-24 keV) energy bands, respectively. " $\mathrm{F}+\mathrm{H}$," for example, refers to sources detected in the full and hard bands only, but not in the soft band, and "S" refers to sources detected exclusively in the soft band. (2) The number of sources detected post-deblending, for a given band or set of bands. (3) The number of sources with spectroscopic redshift measurements.

Deblending is performed following the procedure outlined in detail in Section 2.3.2 of M15. In short, for a given source, the contributions from neighboring detections (within a 90" radius) to the source aperture counts are accounted for using knowledge of their separation and brightness. The false probabilities and photometric quantities (e.g., counts, flux) are all recalculated post-deblending and included in the catalog in separate columns. Out of the total 498 sources in the source catalog, only one is no longer significant (according to our detection threshold) post-deblending.

NUSTAR hard-to-soft-band ratios $\left(\mathrm{BR}_{\mathrm{Nu}}\right)$ are calculated as the ratio of the 8-24 to $3-8 \mathrm{keV}$ count rates. For sources with fullband counts of $S_{\text {net }}>100$, and with a detection in at least one of the soft or hard bands, we derive an effective photon index $\left(\Gamma_{\text {eff }}\right)$, i.e., the spectral slope of a power-law spectrum that is required to produce a given band ratio.

To measure fluxes, we convert from the deblended $30^{\prime \prime}$ count rates using the following conversion factors: $6.7 \times 10^{-11}$, $9.4 \times 10^{-11}$, and $13.9 \times 10^{-11} \mathrm{erg} \mathrm{cm}^{-2} \mathrm{cts}^{-1}$ for the soft, full, and hard bands, respectively. These conversion factors were derived to account for the NuSTAR response, and they assume an unabsorbed power law with a photon index of $\Gamma_{\text {eff }}=1.8$ (typical of AGNs detected by NuSTAR; e.g., Alexander et al. 2013). The conversion factors return aperture-corrected fluxes, i.e., they are corrected to the $100 \%$ encircled-energy fraction of the PSF. The general agreement between our NuSTAR fluxes and those from Chandra and XMM-Newton (see Section 3.1) indicates that the NUSTAR flux measurements are reliable. For sources with spectroscopic redshifts, we determine the rest-frame $10-40 \mathrm{keV}$ luminosity by extrapolating from a measured observed-frame flux, assuming a photon index of $\Gamma_{\text {eff }}=1.8$. To ensure that the adopted observed-frame flux energy band corresponds to the rest-frame $10-40 \mathrm{keV}$ energy band, we use the observed-frame 8-24 and 3-8 keV bands for sources with redshifts of $z<1.35$ and $z \geqslant 1.35$, respectively. For cases with a nondetection in the relevant band (i.e., $8-24$ or $3-8 \mathrm{keV}$ ), we instead extrapolate from the full band $(3-24 \mathrm{keV})$.

\subsection{The Source Catalog}

The serendipitous survey source catalog is provided online in machine-readable format. In Appendix A.1 we give a detailed description of the 106 columns that are provided in the catalog. In total, the catalog contains 497 sources that are significantly detected (according to the definition in Section 2.3) post-deblending, in at least one energy band. Table 2 provides source detection statistics, broken down for the different combinations of energy bands, and the number of sources with spectroscopic redshift measurements.

In addition to the primary source detection approach (Section 2.3), which has been used to generate the above main catalog, in Appendix A.6 we provide a "secondary catalog" containing sources that do not appear in the main catalog (for reasons described therein). However, all results in this work are limited to the main catalog only (the secondary catalog is thus briefer in content).

\section{The Multiwavelength Data}

The positional accuracy of $N U S T A R$ ranges from $\approx 8^{\prime \prime}$ to $\approx 20^{\prime \prime}$, depending on the source brightness (the latter is demonstrated in the following section). For matching to unique counterparts at other wavelengths (e.g., optical and IR), a higher astrometric accuracy is required, especially toward the Galactic plane, where the sky density of sources increases dramatically. We therefore first match to soft X-ray (Chandra, XMM-Newton, and Swift XRT) counterparts, which have significantly higher positional accuracy (Section 3.1), before proceeding to identify optical and IR counterparts (Section 3.2) and undertaking optical spectroscopy (Section 3.3).

\subsection{Soft X-Ray Counterparts}

The NUSTAR serendipitous survey is mostly composed of fields containing well-known extragalactic and Galactic targets. This means that the large majority of the serendipitous survey sources also have lower-energy (or "soft") X-ray coverage from Chandra, XMM-Newton, or Swift XRT, thanks to the relatively large FOVs of these observatories. In addition, short-exposure coordinated Swift XRT observations have been obtained for the majority of the NuSTAR observations. Overall, 81\% (401/497) of the NUSTAR detections have coverage with Chandra or XMM-Newton, and this increases to $99 \%(493 / 497)$ if Swift XRT coverage is included. Only 1\% (4/497) lack any form of coverage from all of these three soft X-ray observatories.

We cross-match with the third XMM-Newton serendipitous source catalog (3XMM; Watson et al. 2009; Rosen et al. 2016) and the Chandra Source Catalog (CSC; Evans et al. 2010) using a $30^{\prime \prime}$ search radius from each $N U S T A R$ source position; the errors in the source matching are dominated by the NuSTAR positional uncertainty (as quantified below). Based on the sky density of X-ray sources with $f_{2-10 \mathrm{keV}} \gtrsim 10^{-14} \mathrm{erg} \mathrm{s}^{-1} \mathrm{~cm}^{-2}$ found by Mateos et al. (2008; for $|b|>20^{\circ}$ sources in the $X M M-N e w t o n$ serendipitous survey), we estimate that the $30^{\prime \prime}$ radius matching results in a typical spurious match fraction of $\approx 7 \%$ for this flux level and latitude range. Overall, we find multiple matches for $\approx 20 \%$ of the cases where there is at least one match. In these multiple-match cases we assume that the 3XMM or CSC source with the brightest hard-band $(4.5-12 \mathrm{keV}$ and $2-7 \mathrm{keV}$, respectively) flux is the correct counterpart. ${ }^{31} \mathrm{We}$ provide the positions and Chandra/XMM-

\footnotetext{
31 For clarity, throughout the paper we refer to the 3-8 keV band as the "soft" band, since it represents the lower (i.e., "softer") end of the energy range for which NUSTAR is sensitive. However, energies of 3-8 keV (and other similar bands, e.g., 2-7 keV) are commonly referred to as "hard" in the context of lower-energy X-ray missions such as Chandra and XMM-Newton, for which these energies are at the upper end of the telescope sensitivity.
} 
Newton $3-8 \mathrm{keV}$ fluxes $\left(F_{\text {soft }}\right)$ for these counterparts in the source catalog (see Appendix A.1). To assess possible flux contributions from other nearby Chandra/XMM-Newton sources, we also determine the total combined flux of all 3XMM or CSC sources contained within the $30^{\prime \prime}$ search aperture $\left(F_{\text {soft }}^{30}\right)$. Of the 284 sources that are successfully matched to $3 \mathrm{XMM}$ or CSC, $29(10 \%)$ have $F_{\text {soft }}^{30}$ values that exceed $F_{\text {soft }}$ by a factor of $>1.2$, and there are only four cases where this factor is $>2$. In other words, there are few cases where additional nearby X-ray sources appear to be contributing substantially to the NUSTAR-detected emission.

In addition to the aforementioned catalog matching, we identify archival Chandra, XMM-Newton, and Swift XRT data that overlap in sky coverage with the NuSTAR data. Using these archival data sets, we manually identify and measure positions for soft X-ray counterparts that are not already included in the 3XMM and CSC catalogs. For Chandra we process the archival data using CHANDRA_REPRO, ${ }^{32}$ for XMMNewton we analyze data products from the Pipeline Processing System, ${ }^{33}$ and for Swift XRT we use screened event files (as provided on HEASARC). ${ }^{34}$ We perform source detection on the archival soft X-ray $(\approx 0.5-8 \mathrm{keV})$ count images using the CIAO source detection algorithm wavdetect (Freeman et al. 2002), which identifies 111 new soft X-ray counterparts. Of these, $88 \%$ have high detection significances (false probabilities of $<10^{-6}$ ), and $12 \%$ have moderate detection significances (false probabilities of $10^{-6}-10^{-4}$ ).

In total, soft X-ray counterparts are successfully identified for $79 \%(395 / 497)$ of the NUSTAR detections: 284 are existing counterparts in the $3 \mathrm{XMM}$ and CSC catalogs, with 269 and 82 counterparts from the individual $3 \mathrm{XMM}$ and CSC catalogs, respectively. Of the remaining $213 \mathrm{NuSTAR}$ detections that lack 3XMM and CSC counterparts, we have manually identified soft $\mathrm{X}$-ray counterparts in archival data (using wavdetect as described above) for 111 sources, of which 27, 60, and 24 are from Chandra, Swift XRT, and XMM-Newton data, respectively. In addition, we manually determine new Chandra positions for 12 sources that appear in $3 \mathrm{XMM}$ and not CSC, but have Chandra coverage, thus improving the X-ray position constraints for these sources. For four of these sources, the newly measured Chandra positions were obtained through our own Chandra observing program aimed at localizing the X-ray emission for Galactic-candidate NuSTAR serendipitous sources (J. A. Tomsick et al. 2017, in preparation). For the soft X-ray counterparts that are detected with multiple soft X-ray observatories, we adopt the position with the highest accuracy: for $31 \%(121 / 395)$ the adopted position is from Chandra, which has the best positional accuracy; for $54 \%(214 / 395)$ the adopted position is from XMM-Newton; and for $15 \%(60 / 395)$ the adopted counterpart is from Swift XRT.

Overall, 21\% (102/497) of the NUSTAR detections lack soft $\mathrm{X}$-ray counterparts. This can largely be explained as a result of insufficient-depth soft X-ray coverage, or zero coverage. However, for the sources with sufficient-depth soft X-ray coverage this may indicate either a spurious NuSTAR detection, a transient detection, or the detection of an unidentified contaminating feature such as stray light (e.g., Mori et al. 2015). We estimate that there are 34 (out of 102) such sources that lack a soft X-ray

\footnotetext{
32 http://cxc.harvard.edu/ciao/ahelp/chandra_repro.html

33 http://www.cosmos.esa.int/web/xmm-newton/pipeline

34 http://heasarc.gsfc.nasa.gov
}
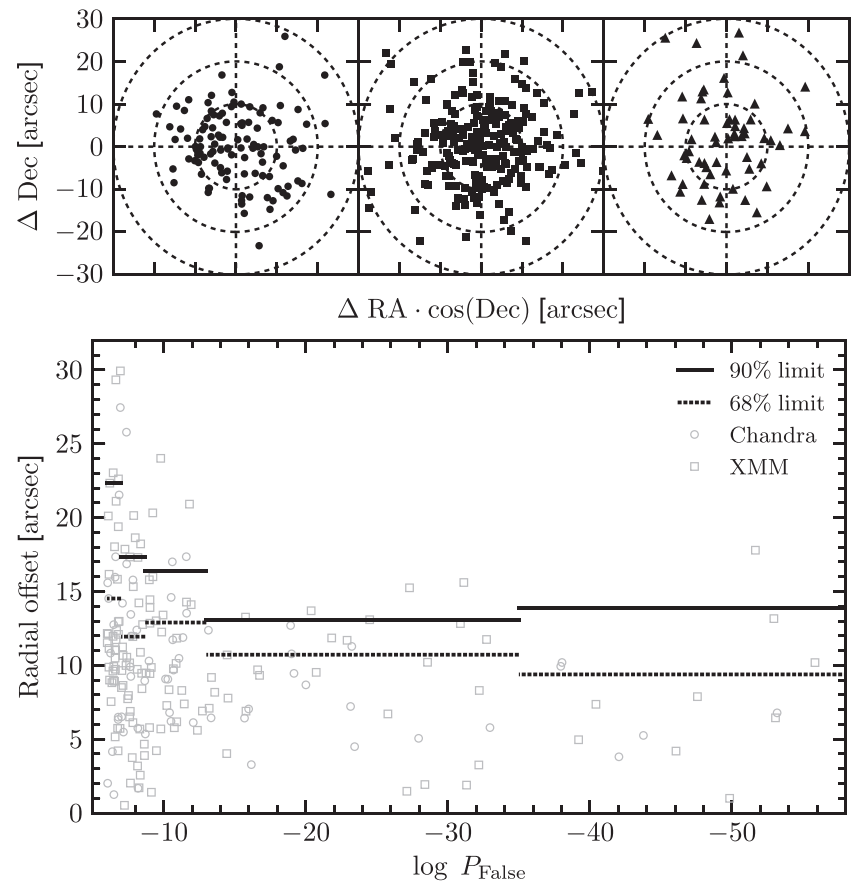

Figure 4. Top panel: astrometric offsets between the NuSTAR coordinates and lower-energy X-ray counterpart coordinates as identified with Chandra (circles, left panel), XMM-Newton (squares, middle panel), and Swift XRT (triangles, right panel). Bottom panel: angular separation between NUSTAR and Chandra/XMM-Newton coordinates, as a function of $P_{\text {False }}$ (source detection significance increases toward the right). The solid and dotted lines show the limits in angular offset enclosing $90 \%$ and $68 \%$ of sources, respectively, for bins in $P_{\text {False }}$. Each bin contains $\approx 40-50$ sources, except the rightmost bin, which contains 23 sources (and extends beyond the $x$-axis upper limit, including all sources with $P_{\text {False }}<-35$ ). This figure illustrates the positional accuracy of NUSTAR as a function of source significance.

counterpart but have sufficiently deep soft X-ray data (from Chandra or XMM-Newton) that we would expect a detection (given the NUSTAR source flux in the overlapping 3-8 keV band). We retain these sources in the sample, but note that their inclusion (or exclusion) has a negligible impact on the results presented herein, which are primarily based on the broader subsample with successful counterpart identifications and spectroscopic redshift measurements.

The top panel of Figure 4 shows the distribution of positional offsets (in R.A. and decl.) for the NuSTAR sources relative to their soft X-ray (Chandra, XMM-Newton, and Swift XRT) counterparts. We find no evidence for systematic differences in the astrometry between observatories, since the mean positional offsets are all consistent with zero: the mean values of $\Delta$ R.A. $\cdot \cos ($ Decl. $)$ and $\Delta$ Decl. are $0 . " 41 \pm 1.45$ and 0 " $18 \pm 1$ ". 28 for Chandra, -0 " $19 \pm 1$ ". 11 and 0 ". $50 \pm 0$ ". 95 for XMM-Newton, and -0 ". $34 \pm 1$ !"97 and 1".70 \pm 2 !"09 for Swift XRT.

The bottom panel of Figure 4 shows the radial separation (in arcseconds) of NuSTAR sources from their well-localized soft $\mathrm{X}$-ray counterparts (for those sources with Chandra or XMMNewton counterparts) as a function of $P_{\text {False }}$, thus illustrating the positional accuracy of $N U S T A R$ as a function of source detection significance. To reliably assess the positional accuracy of $N U S T A R$, we limit this particular analysis to sources with unique matches at soft X-ray energies, and thus with higher likelihoods of being correctly matched. Assuming zero uncertainty on the Chandra and XMM-Newton positions, the $90 \%$ confidence limit on the NuSTAR positional uncertainty 


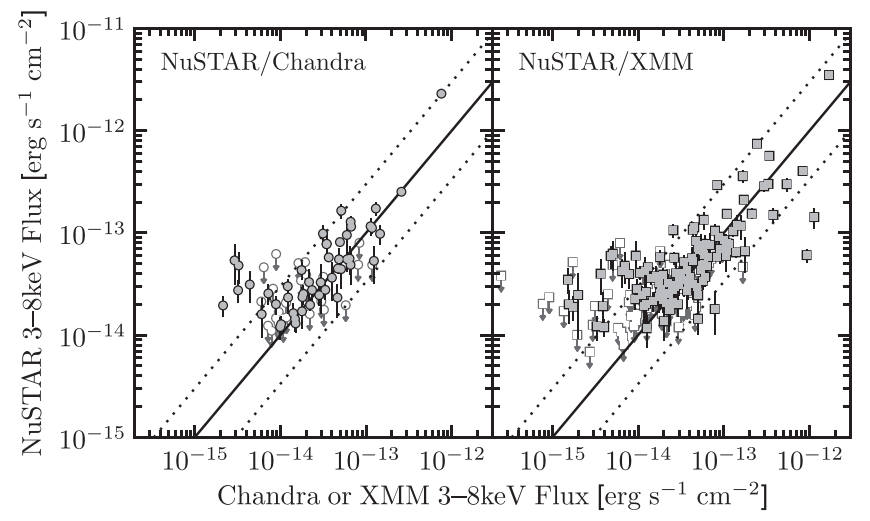

Figure 5. Comparison of the NUSTAR and soft X-ray mission flux ( $F_{\text {soft }}$; from Chandra or XMM-Newton), at 3-8 keV, for those serendipitous survey sources with matched CSC or 3XMM counterparts. The open symbols show upper limits. The 1:1 relation is shown by a solid line, and the dotted lines show values a factor of three from this relation.

is $22^{\prime \prime}$ for the least significant detections and $14^{\prime \prime}$ for the most significant detections. If we instead only consider the Chandra positions, which are in general more tightly constrained (positional accuracy $\lesssim 1^{\prime \prime}$; e.g., see Section 3.2), then the inferred $90 \%$ positional accuracy of NUSTAR improves to $20^{\prime \prime}$ and $12^{\prime \prime}$ for the least significant and most significant sources, respectively.

Figure 5 compares the $3-8 \mathrm{keV}$ fluxes, as measured by NuSTAR, with those measured by Chandra and XMM-Newton for the sources with $3 \mathrm{XMM}$ or CSC counterparts. The small flux corrections from the 3XMM and CSC energy bands (4.5-12 keV and 2-7 keV, respectively) to the 3-8 keV energy band are described in Appendix A.1. The majority of sources ( $92 \%$ and $89 \%$ for Chandra and XMM-Newton, respectively) are consistent with lying within a factor of three of the 1:1 relation, given the uncertainties, and thus show reasonable agreement between observatories. Given that the NuSTAR and the lower-energy X-ray observations are not contemporaneous, intrinsic source variability is expected to contribute to the observed scatter. A number of sources at the lowest X-ray fluxes lie above the relation, due to Eddington bias. This effect has been observed in the NuSTAR-ECDFS and NuSTARCOSMOS surveys (M15; C15) and is predicted from simulations (C15).

Two relatively high flux 3XMM sources lie significantly below the $1: 1$ relation, suggesting that they have experienced a large decrease in flux (by a factor of $\gtrsim 5$ ). The first, NuSTAR J1834520845.6, is a known Galactic magnetar for which the NuSTAR (2015) flux is lower than the XMM-Newton (2005 and 2011 combined) flux by a factor of $\approx 15$. This is broadly consistent with the known properties of the source, which varies in X-ray flux by orders of magnitude over multiyear timescales (e.g., Younes et al. 2012). The second outlying source is extragalactic in origin: NuSTAR J133311-3406.8 (hereafter J1333; $z=0.091$, $L_{10-40 \mathrm{keV}}=8 \times 10^{42} \mathrm{erg} \mathrm{s}^{-1}$ ). Our NTT (ESO New Technology Telescope) spectrum for J1333 reveals a narrow-line AGN (NLAGN), with an apparently asymmetric, blue-wing component to the $\mathrm{H} \alpha+[\mathrm{N}$ II] complex, and our NTT $R$-band imaging shows a well-resolved, undisturbed host galaxy. Modeling the $X M M$ Newton (14 ks exposure; $\approx 1100$ EPIC source counts at $0.5-10 \mathrm{keV})$ and NuSTAR (17 ks exposure; $\approx 75$ source counts at 3-24 keV) spectra, the former of which precedes the latter by $\approx 9 \mathrm{yr}$, the X-ray spectral flux has decreased by a factor of $\approx 5$ in the energy band where the observatories overlap in sensitivity $(3-10 \mathrm{keV})$. While this is an outlier, it is not unexpected to observe one AGN with this level of variability, given the range of AGN variability observed on decade timescales in deep $<10 \mathrm{keV}$ $\mathrm{X}$-ray surveys such as CDFS. The variability could be due to a change in the intrinsic X-ray continuum or the line-of-sight column density (e.g., Risaliti et al. 2005; Markowitz et al. 2014). However, it is not possible to place informative constraints on spectral shape variability of $\mathrm{J} 1333$, since the $N U S T A R$ spectral shape is poorly constrained at $3-10 \mathrm{keV}\left(\Gamma_{\mathrm{eff}}=1.2_{-1.7}^{+1.3}\right)$. Deeper, simultaneous broadband X-ray coverage would be required to determine whether a variation in spectral shape accompanies the relatively large variation in AGN flux. There is Swift XRT coverage contemporaneous with the 2014 NuSTAR data, but J1333 is undetected by Swift XRT. The Swift XRT flux upper limit is consistent with the NuSTAR flux and is a factor of $\approx 4.2$ lower than the XMM-Newton flux (and thus in agreement with a factor of $\approx 5$ variation in the X-ray flux). This source represents the maximum variation in AGN flux identified in the survey.

\subsection{IR and Optical Counterparts}

Here we describe the procedure for matching between the 395 (out of 497) NuSTAR sources with soft X-ray counterparts (identified in Section 3.1) and counterparts at IR and optical wavelengths. The results from this matching are summarized in Table 3 (for the sources with Galactic latitudes of $|b|>10^{\circ}$ ). We adopt matching radii that are a compromise between maximizing completeness and minimizing spurious matches, and we take into account the additional uncertainty (at the level of $1^{\prime \prime}$ ) between the X-ray and the optical/IR positions. For Chandra positions we use a matching radius of $2 . " 5$, which is well motivated based on the known behavior of the positional uncertainty as a function of off-axis angle (the majority of NUSTAR serendipitous sources lie significantly off-axis) and source counts (e.g., Alexander et al. 2003; Lehmer et al. 2005; Xue et al. 2011). For Swift XRT positions we use a matching radius of 6 ", justified by the typical positional uncertainty (statistical plus systematic), which is at the level of $\approx 5$ !" $5(90 \%$ confidence level; e.g., Moretti et al. 2006; Evans et al. 2014). For XMM-Newton positions we use a matching radius of $5^{\prime \prime}$, which is motivated by the typical positional uncertainties of $\mathrm{X}$-ray sources in the XMM-Newton serendipitous survey (e.g., $\approx 4^{\prime \prime}$ at the $90 \%$ confidence level for XMM-Newton bright serendipitous survey sources; Caccianiga et al. 2008).

To identify IR counterparts, we match to the WISE all-sky survey catalog (Wright et al. 2010). Of the 395 sources with soft X-ray counterparts, $274(69 \%)$ have WISE matches. In $100 \%$ of these cases there is a single unique WISE match (detected in at least one WISE band). To identify optical counterparts, we match to the SDSS DR7 catalog (York et al. 2000) and the USNOB1 catalog (Monet et al. 2003). If both contain matches, we adopt optical source properties from the former catalog. Of the 395 sources with soft X-ray counterparts, $252(64 \%)$ have a match in at least one of these optical catalogs: 121 have an SDSS match and 131 without SDSS matches have a USNOB1 match. In 77\% (193/252) of cases there is a single optical match. In the case of multiple matches within the search radius we adopt the closest source. Figure 6 shows the distribution of astrometric offsets between the soft X-ray counterparts and the WISE and optical (SDSS and USNOB1) counterparts. For Galactic latitudes of $|b|>10^{\circ}$, which we focus on for the analysis of $N u S T A R$ 
Table 3

Summary of the Optical and IR Counterpart Matching Statistics and Photometric Magnitudes

\begin{tabular}{lcccccc}
\hline \hline $\begin{array}{l}\text { Catalog/Band } \\
(1)\end{array}$ & $\begin{array}{c}N \\
(2)\end{array}$ & $\begin{array}{c}\text { Fraction } \\
(3)\end{array}$ & $\begin{array}{c}m_{\max } \\
(4)\end{array}$ & $\begin{array}{c}m_{\min } \\
(5)\end{array}$ & $\begin{array}{c}\bar{m} \\
(6)\end{array}$ & $\begin{array}{c}\langle m\rangle \\
(7)\end{array}$ \\
\hline Total optical + IR & 290 & $87.9 \%$ & $\ldots$ & $\ldots$ & $\ldots$ & $\ldots$ \\
WISE (all) & 252 & $76.4 \%$ & $\ldots$ & $\ldots$ & $\ldots$ & $\ldots$ \\
$W I S E / W 1$ & 249 & $75.5 \%$ & 18.4 & 7.8 & 15.3 & 15.5 \\
$W I S E / W 2$ & 248 & $75.2 \%$ & 17.1 & 7.9 & 14.4 & 14.6 \\
WISE $/ W 3$ & 194 & $58.8 \%$ & 13.3 & 4.5 & 11.2 & 11.4 \\
WISE $/ W 4$ & 131 & $39.7 \%$ & 9.9 & 1.8 & 8.1 & 8.4 \\
Optical (all) & 249 & $75.5 \%$ & $\ldots$ & $\ldots$ & $\ldots$ & $\ldots$ \\
SDSS $/ r$ & 121 & $36.7 \%$ & 24.5 & 11.7 & 19.6 & 19.9 \\
USNOB1/R & 198 & $60.0 \%$ & 20.9 & 10.5 & 18.5 & 19.1 \\
\hline
\end{tabular}

Notes. Summary of the optical and IR counterpart matching for the 330 NuSTAR serendipitous survey sources with high Galactic latitudes $\left(|b|>10^{\circ}\right)$ and soft X-ray telescope (Chandra, Swift XRT, or XMM-Newton) counterpart positions (see Section 3.2). Columns: (1) The catalog and photometric band (where magnitude statistics are provided). (2) The number of the NuSTAR sources successfully matched to a counterpart in a given catalog. For the WISE all-sky survey catalog, this is broken down for the four photometric WISE bands. (3) The fraction of the NUSTAR sources that are matched. (4) The maximum (i.e., faintest) magnitude for the counterparts in a given catalog and photometric band. (5) The minimum (i.e., brightest) magnitude. (6) The mean magnitude. (7) The median magnitude.

serendipitous survey source properties (Section 4), the spurious matching fractions are low ( $\lesssim 10 \%$; see Appendix A.3).

For the 143 (out of 395) soft X-ray counterparts without SDSS and USNOB1 matches, we determine whether there are detections within the existing optical coverage, which is primarily photographic plate coverage (obtained through the DSS) but also includes dedicated $R$-band and multiband imaging from our own programs with the ESO-NTT (EFOSC2) and ESO-2.2 $\mathrm{m}$ (GROND), respectively. This identifies an additional 33 optical counterparts. For the 110 nondetections, we estimate $R$-band magnitude lower limits from the data (all cases have coverage, at least from photographic plate observations). These optical nondetections do not rule out follow-up spectroscopy; for 21 of them we have successfully performed optical spectroscopy, obtaining classifications and redshifts, either by identifying an optical counterpart in preimaging or by positioning the spectroscopic slit on a WISE source within the X-ray error circle. In Figure 7 we show histograms of the WISE and $R$-band magnitudes for the NUSTAR sources with soft X-ray counterparts.

For the 102 (out of 497) sources without soft X-ray counterparts, the X-ray positional error circle (from NuSTAR) is comparatively large (see Section 3.1), so unique counterparts cannot be identified with high confidence. To identify possible counterparts for these sources, for the purposes of optical spectroscopic follow-up, we consider the properties of nearby WISE sources. Matching to the WISE all-sky survey, we identify AGN candidates within a $25^{\prime \prime}$ radius of the NUSTAR position, using the following two criteria: a WISE color of $W 1-W 2>0.8$ (and $W 2<15$; Vega mag; Stern et al. 2012) or a $W 4$-band detection. The WISE W1,W2,W3, and $W 4$ bands are are centered at $3.4,4.6,12$, and $22 \mu \mathrm{m}$, respectively. We limit this matching to the 85 (out of 102) sources at Galactic latitudes above $|b|=10^{\circ}$. Given the sky densities of WISE sources that satisfy these criteria $\left(\approx 46 \mathrm{deg}^{-2}\right.$ and $\approx 730 \mathrm{deg}^{-2}$, respectively, for $|b|>10^{\circ}$ ), the probabilities of chance matches

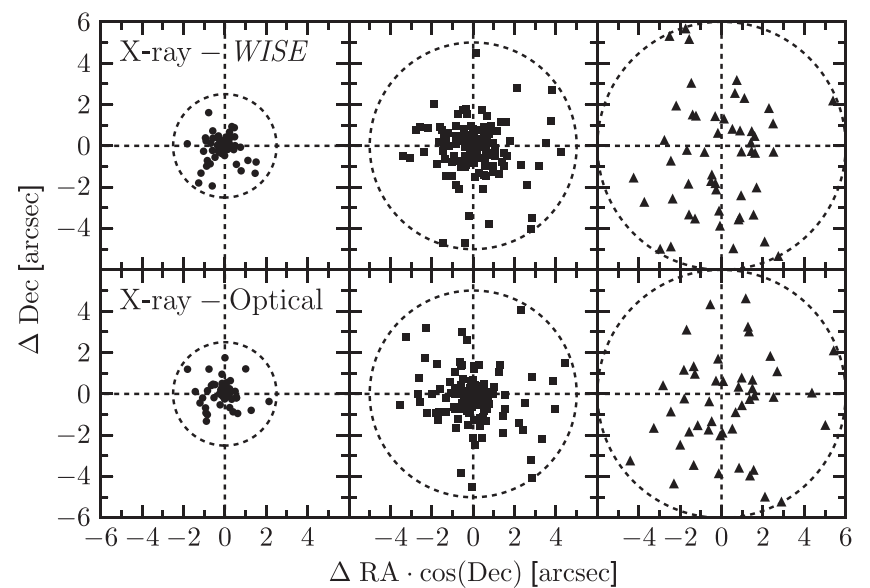

Figure 6. Astrometric offsets between the soft X-ray counterpart coordinates and the WISE (top row) and optical (bottom row) coordinates. The soft X-ray counterparts are from Chandra (left column), XMM-Newton (middle column), and Swift XRT (right column). The dashed circles correspond to the search radii for each telescope $\left(2 " .5,5^{\prime \prime}\right.$, and $6^{\prime \prime}$ for Chandra, XMM-Newton, and Swift XRT, respectively).

are $\approx 1 \%$ and $\approx 11 \%$, respectively. Where multiple such WISE sources are identified, we prioritize those that satisfy both criteria, then those that satisfy the former criterion. For 24 (out of 102) of these sources there is a WISE AGN candidate within the NUSTAR error circle, the position of which we match to optical counterparts. The optical and IR counterparts identified in this manner (for NuSTAR sources without soft X-ray counterparts) are primarily used for the purposes of undertaking spectroscopic follow-up (Section 3.3), and we exclude them from our analysis of the IR properties of the NuSTAR serendipitous survey AGNs (Section 4.3), to avoid biasing the results. For the remaining 78 (out of 102) sources at $|b|<10^{\circ}$ or without matches to WISE AGN candidates, we use the available $R$-band information to obtain magnitude constraints: in cases where there is at least one optical source within the $N U S T A R$ error circle, we adopt the lowest (i.e., brightest) $R$ band magnitude as a lower limit, and in cases with no optical source within the NuSTAR error circle, we adopt the magnitude limit of the imaging data.

For a large fraction of the sources discussed in this section, the spectroscopic follow-up (Section 3.3) shows evidence for an AGN, which provides additional strong support for correct counterpart identification (given the low sky density of AGNs). Furthermore, the optical and IR photometric properties of the NUSTAR serendipitous survey counterparts are in agreement with AGNs (see Sections 4.2.1 and 4.3.1).

\subsection{Optical Spectroscopy}

Optical identifications and source redshifts, obtained through spectroscopy, are a prerequisite to the measurement of intrinsic source properties such as luminosity and the amount of obscuration. A small fraction $(\approx 11 \% ; 57 / 497)$ of the $N u S T A R$ serendipitous survey sources have preexisting spectroscopic coverage, primarily from the SDSS. However, the majority $(\approx 89 \%)$ of the serendipitous survey sources do not have preexisting spectroscopy. For that reason, we have undertaken a campaign of dedicated spectroscopic follow-up in the optical-IR bands (Section 3.3.1), obtaining spectroscopic identifications for a large fraction $(56 \%)$ of the total sample. 

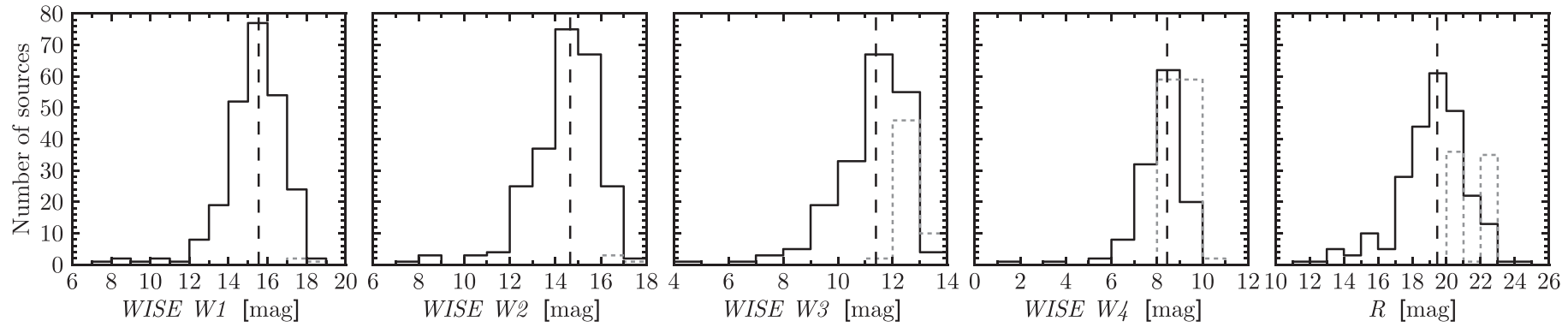

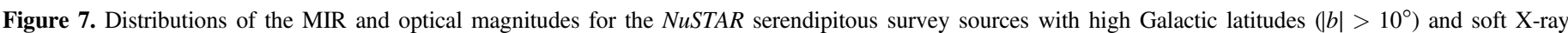

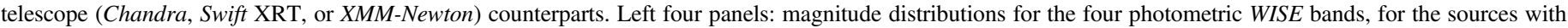

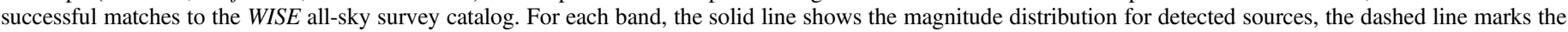

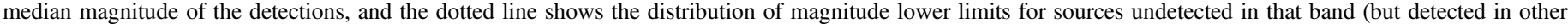
bands). Right panel: $R$-band magnitudes (primarily from matching to the SDSS and USNOB1 catalogs) for all the sources.

For the high Galactic latitude $\left(|b|>10^{\circ}\right)$ samples selected in individual bands, this has resulted in a spectroscopic completeness of $\approx 70 \%$. The analysis of and classifications obtained from these new spectroscopic data and those from preexisting spectroscopy are described in Section 3.3.2.

\subsubsection{Dedicated Follow-up Campaign}

Since NUSTAR performs science pointings across the whole sky, a successful ground-based follow-up campaign requires the use of observatories at a range of geographic latitudes, and preferably across a range of dates throughout the sidereal year. This has been achieved through observing programs with, primarily, the following telescopes over a multiyear period: the Hale Telescope at Palomar Observatory $\left(5.1 \mathrm{~m}\right.$; decl. $\gtrsim-21^{\circ}$; PIs F. A. Harrison and D. Stern); Keck I and II at the W. M. Keck Observatory $\left(10 \mathrm{~m} ;-35^{\circ} \lesssim\right.$ decl. $\lesssim 75^{\circ}$; PIs F. A. Harrison and D. Stern); the New Technology Telescope (NTT) at La Silla Observatory $\left(3.6 \mathrm{~m}\right.$; decl. $\lesssim 25^{\circ}$; PI G. B. Lansbury); ${ }^{35}$ the Magellan I (Baade) and Magellan II (Clay) Telescopes at Las Campanas Observatory $(6.5 \mathrm{~m}$; decl. $\lesssim 25^{\circ} ;$ PIs E. Treister and F. E. Bauer); ${ }^{36}$ and the Gemini-South observatory $\left(8.1 \mathrm{~m}\right.$; decl. $\lesssim 25^{\circ}$; PI E. Treister). ${ }^{37}$ Table 4 provides a list of the observing runs undertaken. In each case we provide the observing run starting date (UT), number of nights, telescope, instrument, and total number of spectroscopic redshifts obtained for NUSTAR serendipitous survey sources.

The total number of sources with spectroscopic redshift measurements and classifications is 276 . The large majority of spectroscopic identifications in the Northern Hemisphere were obtained using a combination of Palomar and Keck, with the former being efficient for brighter targets and the latter for fainter targets. These account for 51\% (141/276) of the spectroscopically identified sample. Similarly, for the Southern Hemisphere the majority of spectroscopic identifications were obtained using the ESO-NTT, while complementary Magellan observations were used to identify the fainter optical sources. These account for $28 \%(76 / 276)$ of the overall spectroscopically identified sample.

Conventional procedures were followed for spectroscopic data reduction, using IRAF routines. Spectrophotometric

\footnotetext{
35 Program IDs: 093.B-0881, 094.B-0891, 095.B-0951, and 096.B-0947.

36 Program IDs: CN2013B-86, CN2014B-113, CN2015A-87, and CN2016A-93.

${ }^{37}$ Program ID: GS-2016A-Q-45.
}

standard star observations, from the same night as the science observations, were used to flux-calibrate the spectra.

\subsubsection{Spectral Classification and Analysis}

All flux-calibrated optical spectra from this work are provided in Appendix A.2. For our instrument setups, the typical observed-frame wavelength range covered is $\lambda \approx$ $3500-9000 \AA$. At lower redshifts, for example $z=0.3$, this results in coverage for the following emission lines common to AGNs and quasars: $\mathrm{Mg}$ II $\lambda 2800$, [Ne V] $\lambda \lambda 3346$ and 3426, [O II] $\lambda 3728,[\mathrm{Ne}$ III] $\lambda 3869, \quad \mathrm{H} \delta \lambda 4102, \mathrm{H} \gamma \lambda 4340$, $\mathrm{H} \beta \lambda 4861$, [O III] $\lambda \lambda 4959$ and 5007, [O I] $\lambda \lambda 6300$ and 6364, [N II] $\lambda \lambda 6548$ and $6584, \mathrm{H} \alpha \lambda 6563$, and [S II] $\lambda \lambda 6716$ and 6731. At higher redshifts, for example, $z=2$, the lines covered are Ly $\lambda 1216$, Si IV $\lambda 1398$, C IV $\lambda 1549$, He II $\lambda 1640$, C III] $\lambda 1909, \mathrm{C} \mathrm{II}] \lambda 2326$, and $\mathrm{Mg}$ II $\lambda 2800$.

To measure spectroscopic redshifts, we identify emission and absorption lines and measure their observed-frame wavelengths using Gaussian profile fitting. To determine the redshift solution, we cross-match the wavelength ratios of the identified lines with a look-up table of wavelength ratios based on the emission and absorption lines observed in AGN and galaxy spectra. The final, precise redshift measurement is then obtained from the Gaussian profile fit to the strongest line. For the large majority of cases there are multiple lines detected, and there is only one valid redshift solution. The lines identified for each individual NuSTAR source are tabulated in Appendix A.2. There are only five sources where the redshift is based on a single line identification (marked with "quality B" flags in Appendix A.2). For four of these, the single emission line detected is identified as Mg II $\lambda 2800$. In all cases this is well justified: $\mathrm{Mg}$ II is a dominant broad line in quasar spectra, and there is a relatively large separation in wavelength between the next strong line blueward of $\mathrm{Mg}$ II (C III] $\lambda 1909)$ and that redward of $\mathrm{Mg}$ II $(\mathrm{H} \beta \lambda 4861)$. This means that $\mathrm{Mg}$ II can be observed in isolation for redshifts of $z \sim 0.8$ in cases where our wavelength coverage is slightly narrower than usual, or if the other lines (e.g., $\mathrm{C}$ III] and $\mathrm{H} \beta$ ) are below the detection threshold. Mg II can also be clearly identifiable in higher signalto-noise ratio data, due to the shape of the neighboring Fe II pseudo-continuum.

We perform optical classifications visually, based on the spectral lines observed. For the extragalactic sources with available optical spectra and with identified lines (253 sources), emission lines are detected for all but one source (where multiple absorption lines are identified). Both permitted 
Table 4

Chronological List of Ground-based Observing Runs for Spectroscopic Follow-up of the NUSTAR Serendipitous Survey

\begin{tabular}{|c|c|c|c|c|}
\hline $\begin{array}{l}\text { Run ID } \\
\text { (1) }\end{array}$ & $\begin{array}{l}\text { UT Start Date } \\
\text { (2) }\end{array}$ & $\begin{array}{l}\text { Telescope } \\
\text { (3) }\end{array}$ & $\begin{array}{l}\text { Instrument } \\
\text { (4) }\end{array}$ & $\begin{array}{c}\text { Spectra } \\
\text { (5) }\end{array}$ \\
\hline 1 & 2012 Oct 10 & Palomar & DBSP & 1 \\
\hline 2 & 2012 Oct 13 & Keck & DEIMOS & 1 \\
\hline 3 & 2012 Nov 09 & Keck & LRIS & 1 \\
\hline 4 & 2012 Nov 20 & Palomar & DBSP & 2 \\
\hline 5 & 2012 Dec 12 & Gemini-South & GMOS & 1 \\
\hline 6 & 2013 Jan 10 & Keck & LRIS & 1 \\
\hline 7 & 2013 Feb 12 & Palomar & DBSP & 2 \\
\hline 8 & 2013 Mar 11 & Palomar & DBSP & 6 \\
\hline 9 & 2013 Jul 07 & Palomar & DBSP & 2 \\
\hline 10 & 2013 Oct 03 & Keck & LRIS & 9 \\
\hline 11 & 2013 Dec 05 & Magellan & MagE & 2 \\
\hline 12 & 2013 Dec 10 & Keck & DEIMOS & 6 \\
\hline 13 & 2014 Feb 22 & Palomar & DBSP & 4 \\
\hline 14 & 2014 Apr 22 & Palomar & DBSP & 6 \\
\hline 15 & 2014 Jun 25 & Keck & LRIS & 12 \\
\hline 16 & 2014 Jun 30 & NTT & EFOSC2 & 8 \\
\hline 17 & 2014 Jul 21 & Palomar & DBSP & 3 \\
\hline 18 & 2014 Sep 25 & Magellan & MagE & 5 \\
\hline 19 & 2014 Oct 20 & Keck & LRIS & 4 \\
\hline 20 & 2014 Dec 23 & Palomar & DBSP & 4 \\
\hline 21 & 2015 Feb 17 & Palomar & DBSP & 5 \\
\hline 22 & 2015 Mar 14 & NTT & EFOSC2 & 17 \\
\hline 23 & 2015 Mar 18 & Magellan & IMACS & 6 \\
\hline 24 & 2015 May 19 & NTT & EFOSC2 & 14 \\
\hline 25 & 2015 Jun 09 & Palomar & DBSP & 1 \\
\hline 26 & 2015 Jun 15 & Palomar & DBSP & 1 \\
\hline 27 & 2015 Jul 17 & Keck & LRIS & 3 \\
\hline 28 & 2015 Jul 21 & Palomar & DBSP & 4 \\
\hline 29 & 2015 Aug 09 & Palomar & DBSP & 6 \\
\hline 30 & 2015 Aug 12 & Keck & LRIS & 6 \\
\hline 31 & 2015 Dec 04 & Keck & LRIS & 28 \\
\hline 32 & 2015 Dec 06 & NTT & EFOSC2 & 25 \\
\hline 33 & 2016 Jan 11 & Palomar & DBSP & 8 \\
\hline 34 & 2016 Feb 05 & Palomar & DBSP & 2 \\
\hline 35 & 2016 Feb 08 & Magellan & MagE & 6 \\
\hline 36 & 2016 Feb 13 & Keck & LRIS & 17 \\
\hline 37 & 2016 Jul 05 & Keck & LRIS & 10 \\
\hline 38 & 2016 Jul 10 & Palomar & DBSP & 6 \\
\hline
\end{tabular}

Notes. Columns: (1) ID assigned to each observing run. (2) Observing run start date. (3) and (4) The telescope and instrument used. (5) The number of spectra from a given observing run that have been adopted, in this work, as the analyzed optical spectrum for a NUSTAR serendipitous survey source. These correspond to the individual sources listed in Table 6 of Appendix A.2 and are primarily $(\approx 93 \%)$ sources with successful redshift measurements and spectroscopic classifications. These source numbers exclude the 35 sources in the secondary catalog for which we have obtained new spectroscopic identifications (see Appendix A.6).

emission lines (e.g., the Balmer series and $\mathrm{Mg}$ II) and forbidden (e.g., [O III] and [N II]) emission lines are identified for 183 (out of 253) sources. For these sources, if any permitted line is broader than the forbidden lines, we assign a broad-line AGN (BLAGN) classification; otherwise, we assign an NLAGN classification. There are 58 (out of 253) sources where only permitted (or semi-forbidden) emission lines are identified. For the majority of these (56 sources) the line profiles are visually broad, and we assign a BLAGN classification (these sources predominantly lie at higher redshifts, with 51 at $z \gtrsim 1$, and have quasar-like continuum-dominated spectra). For 24 sources where there is a level of ambiguity as to whether the permitted lines are broad or not, we append the optical classification (i.e., "NL" or "BL" in Table 6) with a "?" symbol. For the remaining 11 sources (out of 253) with only forbidden-line detections and the single source with absorption-line detections only, we assign NLAGN classifications.

In total we have spectroscopic classifications for 276 of the NUSTAR serendipitous survey sources, including the 253 extragalactic sources mentioned above, an additional BL Lac type object, 16 Galactic $(z=0)$ objects, and 6 additional (BLAGN and NLAGN) classifications from the literature. Of these classifications, 222 were assigned using data from the dedicated observing runs (Table 4), and 54 were assigned using existing data (primarily SDSS) or the literature. Considering the total classified sample, the majority of the sources $(162$, or $58.7 \%)$ are BLAGNs, $97(35.1 \%)$ are NLAGNs, $1(0.4 \%)$ is a BL Lac type object, and the remaining $16(5.8 \%)$ are Galactic objects (e.g., cataclysmic variables and high-mass X-ray binaries). J. A. Tomsick et al. (2017, in preparation) will present a detailed analysis of the Galactic subsample. The current spectroscopic completeness (i.e., the fraction of sources with successful spectroscopic identifications) is $\approx 70 \%$ for the overall serendipitous survey (for the $|b|>10^{\circ}$ individual bandselected samples), although the completeness is a function of X-ray flux (see Section 4.2).

In Table 6 (see Appendix A.2) we provide the following for all NuSTAR serendipitous survey sources with optical spectra: the spectroscopic redshift, the optical classification, the identified emission and absorption lines, individual source notes, and the observing run ID (linking to Table 4).

\section{Results and Discussion}

Here we describe the properties of the NUSTAR serendipitous survey sources, with a focus on the high-energy X-ray (Section 4.1), optical (Section 4.2), and infrared (Section 4.3) wavelength regimes. We compare and contrast with other relevant samples, including the blank-field NuSTAR surveys in well-studied fields (COSMOS and ECDFS); non-survey samples of extreme objects targeted with NuSTAR; the Swift BAT all-sky survey, one of the most sensitive high-energy X-ray surveys to precede NuSTAR; and lower-energy ( $<10 \mathrm{keV}$; e.g., Chandra and XMM-Newton) X-ray surveys.

\subsection{X-Ray Properties}

\subsubsection{Basic NuSTAR Properties}

Overall there are 497 sources with significant detections (post-deblending) in at least one band. Section 2.5 details the source detection statistics, broken down by energy band. In the $8-24 \mathrm{keV}$ band, which is unique to NUSTAR among focusing $\mathrm{X}$-ray observatories, there are 163 detections, i.e., $33 \%$ of the sample. The NuSTAR-COSMOS and NuSTAR-ECDFS surveys found fractions of $8-24 \mathrm{keV}$ detected sources that are consistent with this: $35 \%$ (32/91 sources; C15) and $39 \%$ (19/49 sources post-deblending; M15), respectively.

The net (cleaned, vignetting-corrected) exposure times per source $\left(t_{\text {net }}\right.$; for the combined FPMA $+\mathrm{B}$ data) have a large range, from 10 to $1500 \mathrm{ks}$, with a median of $60 \mathrm{ks}$. For the 3-8 $\mathrm{keV}, 8-24 \mathrm{keV}$, and 3-24 keV bands, the lowest net source counts $\left(S_{\text {net }}\right)$ for sources with detections in these bands are 12, 15 , and 18, respectively. The highest $S_{\text {net }}$ values are 9880, 5853, and 15693, respectively, and correspond to one individual source NuSTAR J043727-4711.5, a BLAGN at 


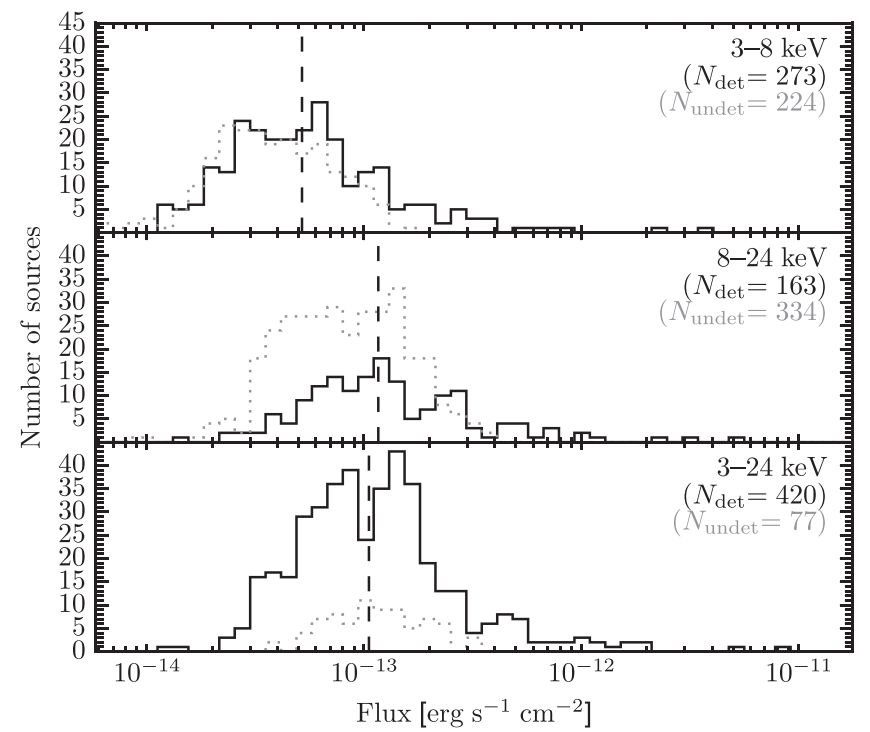

Figure 8. Flux distributions in the soft, hard, and full energy bands (top, middle, and bottom panels, respectively) for the NUSTAR serendipitous survey sample. For each band, the solid line shows the flux distribution for sources independently detected in that band (the number of these sources, $N_{\text {det }}$, is shown in black font), and the median flux of the detected sources is marked by a dashed line. For each band, the dotted line shows the distribution of flux upper limits for sources undetected in that band, but independently detected in at least one other band (the number of these sources, $N_{\text {undet }}$, is shown in gray font).

$z=0.051$. The median $S_{\text {net }}$ values are 56,62 , and 75 , respectively. The count rates are in the range of $0.17-52$ $\mathrm{ks}^{-1}, 0.11-36 \mathrm{ks}^{-1}$, and $0.13-94 \mathrm{ks}^{-1}$, respectively, and the median count rates are $0.77,0.84$, and $1.1 \mathrm{ks}^{-1}$, respectively.

Figure 8 shows the distribution of fluxes for the full sample, for each energy band. The distributions for detected and undetected sources (for a given band) are shown separately. For sources that are detected in the $3-8 \mathrm{keV}, 8-24 \mathrm{keV}$, and $3-24 \mathrm{keV}$ bands, the faintest fluxes measured are 1.17, 1.53 , and $1.22 \times 10^{-14} \mathrm{erg} \mathrm{s}^{-1} \mathrm{~cm}^{-2}$, respectively. The brightest fluxes are 3.5, 5.0, and $8.8 \times 10^{-12} \mathrm{erg} \mathrm{s}^{-1} \mathrm{~cm}^{-2}$, respectively, and correspond to one individual source NuSTAR J075800 +3920.4 , a BLAGN at $z=0.095$. The median fluxes are 5.2, 11.6, and $10.5 \times 10^{-14} \mathrm{erg} \mathrm{s}^{-1} \mathrm{~cm}^{-2}$, respectively. The dynamic range of the serendipitous survey exceeds the other NUSTAR extragalactic survey components. For comparison, the blank-field ECDFS and COSMOS components span $3-24 \mathrm{keV}$ flux ranges of $\approx(2-10) \times 10^{-14} \mathrm{erg} \mathrm{s}^{-1} \mathrm{~cm}^{-2}$ and (5-50) $\times 10^{-14} \mathrm{erg} \mathrm{s}^{-1} \mathrm{~cm}^{-2}$, respectively (C15 and M15). The serendipitous survey pushes to fluxes (both flux limits and median fluxes) roughly two orders of magnitude fainter than those achieved by previous-generation hard X-ray observatories such as Swift BAT (e.g., Baumgartner et al. 2013) and INTEGRAL (e.g., Malizia et al. 2012).

\subsubsection{Band Ratios}

Figure 9 shows the $8-24 \mathrm{keV}$ to $3-8 \mathrm{keV}$ band ratios $\left(\mathrm{BR}_{\mathrm{Nu}}\right)$ for the full sample of NuSTAR serendipitous survey sources, as a function of full-band $(3-24 \mathrm{keV})$ count rate. In order to examine the results for extragalactic sources only, we remove sources that are spectroscopically confirmed as having $z=0$ (see Section 3.3) and exclude sources with Galactic latitudes below $|b|=10^{\circ}$, for which there is significant contamination to the nonspectroscopically identified sample from Galactic sources. A large and statistically significant variation in $\mathrm{BR}_{\mathrm{Nu}}$

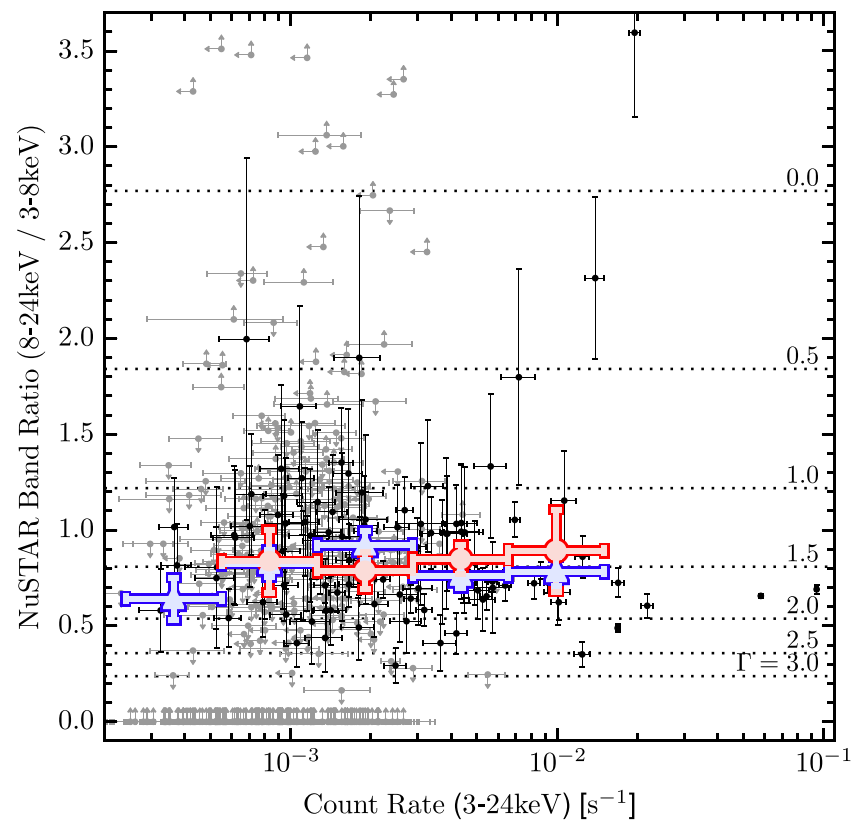

Figure 9. NuSTAR $8-24 \mathrm{keV}$ to $3-8 \mathrm{keV}$ band ratio $\left(\mathrm{BR}_{\mathrm{Nu}}\right)$ vs. full-band (3-24 keV) count rate for the full NUSTAR serendipitous survey sample. Constrained $\mathrm{BR}_{\mathrm{Nu}}$ values are shown in black, and those with upper or lower limits are shown in gray. The dotted horizontal lines indicate spectral slopes $\left(\Gamma_{\text {eff }}\right)$ to which the band ratios correspond. The overplotted red circles show numerical means (binning in full-band count rate), for a subset of extragalactic sources with $\log \left(P_{\text {False }}\right)<-14$. The overplotted blue triangles show "stacked" means obtained from summing the net count rates of all sources, including nondetections, and bootstrapping errors.

is observed across the sample, with some sources exhibiting extreme spectral slopes ( $\Gamma_{\text {eff }} \approx 3$ at the softest values; $\Gamma_{\text {eff }} \approx 0$ at the hardest values).

In Figure 9, we overlay mean band ratios and corresponding errors (in bins of full-band count rate, with an average of 13 sources per bin) for a subset of the extragalactic serendipitous sample with $\log \left(P_{\text {False }}\right)<-14$ in the full band. This cut in source significance reduces the fraction of sources with upper or lower limits in $\mathrm{BR}_{\mathrm{Nu}}$ to only $7 \%$, allowing numerical means to be estimated. The results are consistent with a flat relation in the average band ratio versus count rate and a constant average effective photon index of $\Gamma_{\text {eff }} \approx 1.5$. This value is consistent with the average effective photon index found from spectral analyses of sources detected in the dedicated NuSTAR surveys of the ECDFS, EGS, and COSMOS fields $\left(\Gamma_{\text {eff }}=1.59 \pm 0.14\right.$; A. Del Moro et al. 2017, in preparation). This hard average spectral slope suggests numerous obscured AGNs within the sample. The mean band ratios disfavor an increase toward lower count rates. This is in apparent disagreement with the recent results of M15 for the NuSTAR-ECDFS survey, which show an increase toward lower count rates, albeit for small source numbers with constrained band ratios. Deep surveys at lower X-ray energies have previously found an anticorrelation between band ratio and count rate for the $0.5-8 \mathrm{keV}$ band (e.g., Della Ceca et al. 1999; Ueda et al. 1999; Mushotzky et al. 2000; Tozzi et al. 2001; Alexander et al. 2003), interpreted as being driven by an increase in the number of absorbed AGNs toward lower count rates. We find no evidence for such an anticorrelation in the higher-energy $3-24 \mathrm{keV}$ band. This may be understood partly as a result of the X-ray spectra of AGNs being less strongly affected by absorption in the highenergy NuSTAR band. 


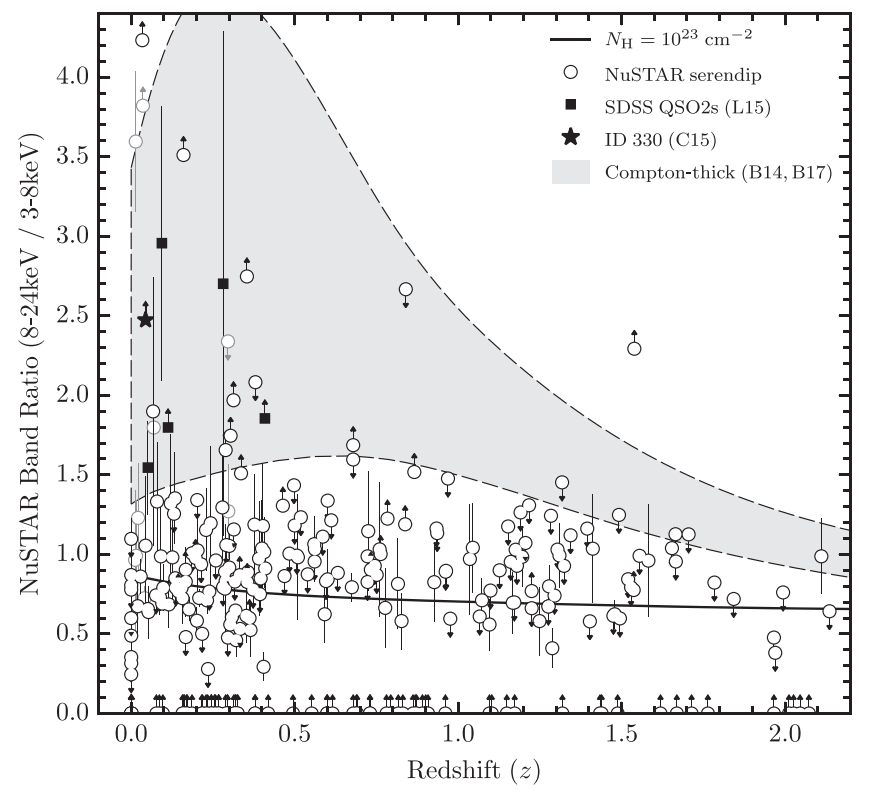

Figure 10. NuSTAR band ratio $\left(\mathrm{BR}_{\mathrm{Nu}}\right)$ vs. redshift $(z)$ for the full $N u S T A R$ serendipitous survey sample (black circles). Sources that are associated with the primary science targets of the NuSTAR observations (according to the $\Delta$ $(c z)$ criterion in Section 2.3) are shown as lighter gray circles. We compare to other NuSTAR-observed sources targeted for other programs (i.e., not part of the serendipitous survey). The black star shows a CT AGN identified in the NuSTAR-COSMOS survey $(\mathrm{C} 15)$. The black squares show heavily obscured SDSS-selected type 2 quasars observed with $N u S T A R$, for which there is evidence for either CT or close to CT absorption (Gandhi et al. 2014; Lansbury et al. 2014, 2015). The gray shaded region highlights the parameter space expected for CT (i.e., $N_{\mathrm{H}}>1.5 \times 10^{24} \mathrm{~cm}^{-2}$ ) AGNs, considering all populations (including reflection- and transmission-dominated CT AGNs), based on results from the NUSTAR snapshot survey (Baloković et al. 2014; M. Baloković et al. 2017, in preparation). This gray region was obtained by redshifting the best-fit spectral models of local CT snapshot AGNs, for which the X-ray spectra are relatively well constrained. The upper and lower extents (dashed lines) represent the 68th percentiles (i.e., 84\% of the CT snapshot AGNs lie above the lower dashed line). Serendipitous sources lying at $\mathrm{BR}_{\mathrm{Nu}}$ values within or higher than this gray region are good candidates for being CT. The black track shows a MYTORUS model prediction for $\mathrm{BR}_{\mathrm{Nu}}$ as a function of redshift, for a more moderate column density of $N_{\mathrm{H}}=10^{23} \mathrm{~cm}^{-2}$.

To incorporate the full serendipitous sample, including weak and nondetections, we also calculate "stacked" means in $\mathrm{BR}_{\mathrm{Nu}}$ (also shown in Figure 9), by summing the net count rates of all sources. The stacked means are also consistent with a flat trend in band ratio as function of count rate.

While obscured AGNs can be crudely identified using $\mathrm{BR}_{\mathrm{Nu}}$ alone, an estimate of obscuring columns requires additional knowledge of the source redshifts, which shift key spectral features (e.g., the photoelectric absorption cutoff) across the observed energy bands. Here we use the combination of $\mathrm{BR}_{\mathrm{Nu}}$ and the source redshifts to identify potentially highly obscured objects. Figure 10 shows $\mathrm{BR}_{\mathrm{Nu}}$ versus $z$ for the spectroscopically identified serendipitous survey sample. We compare with the band ratios measured for CT, or near-CT, SDSSselected type 2 quasars observed with NUSTAR in a separate targeted program (Gandhi et al. 2014; Lansbury et al. 2014, 2015), and with tracks (gray region) predicted for CT absorption based on redshifting the best-fit spectra of local CT AGNs from the NuSTAR snapshot survey of Swift BAT AGNs (Baloković et al. 2014; M. Baloković et al. 2017, in preparation). A number of sources stand out as CT candidates based on this analysis. While $\mathrm{BR}_{\mathrm{Nu}}$ can only provide a crude estimate of the absorbing columns, a more detailed

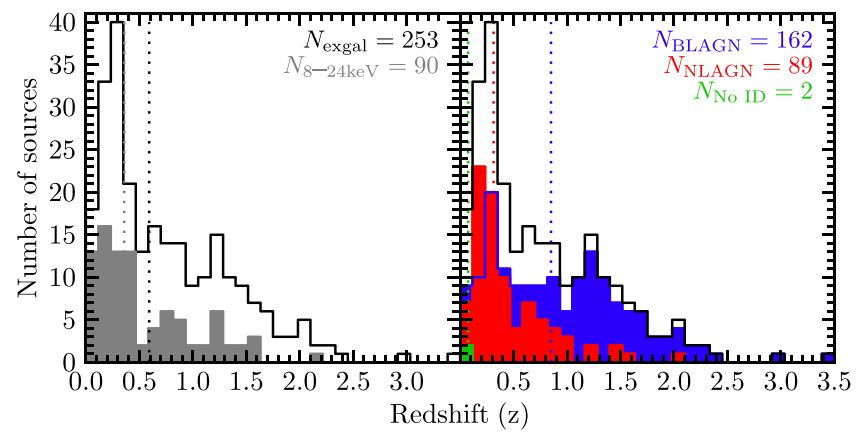

Figure 11. Distribution of spectroscopic redshifts for the spectroscopically identified NuSTAR serendipitous survey sample. Galactic $(z=0)$ sources have been excluded. In addition to the total distribution (black line), the left panel shows the distribution for the subset that are independently detected in the hard band (8-24 keV; gray filled histogram), and the right panel shows the distribution separated by optical classification: BLAGNs are shown in blue; NLAGNs are shown in red. The vertical lines mark the median redshifts.

investigation of the NuSTAR spectra and multiwavelength properties of the CT candidates can strengthen the interpretation of these high-BR $\mathrm{N}_{\mathrm{Nu}}$ sources as highly absorbed systems $(\mathrm{G}$. B. Lansbury et al. 2017, in preparation).

\subsubsection{Redshifts and Luminosities}

Of the NUSTAR serendipitous survey sources with optical spectroscopic coverage and spectroscopic redshift measurements (described in Section 3.3), there are 262 identified as extragalactic. Figure 11 shows the redshift distribution for the extragalactic sources, excluding nine sources with evidence for being associated with the NuSTAR targets for their respective observations (see Section 2.3). The redshifts cover a large range, from $z=0.002$ to 3.43 , with a median of $\langle z\rangle=0.56$. For the 90 extragalactic objects with independent detections in the high-energy band $(8-24 \mathrm{keV})$, to which $N u S T A R$ is uniquely sensitive, the median redshift is $\langle z\rangle=0.34$. Roughly comparable numbers of NLAGNs and BLAGNs are identified for lower redshifts $(z \lesssim 1)$, but there is a significant bias toward BLAGNs at higher redshifts. This was also found for the NuSTAR surveys in well-studied fields (e.g., C15), and for surveys with sensitive lower-energy $(<10 \mathrm{keV}) \mathrm{X}$-ray observatories such as Chandra and XMM-Newton (e.g., Barger et al. 2003; Eckart et al. 2006; Barcons et al. 2007). This effect is largely due to selection biases against the detection of highly absorbed AGNs and against the spectroscopic identification of the optically fainter NLAGNs (e.g., Treister et al. 2004).

Figure 12 shows the redshift-luminosity plane for the restframe $10-40 \mathrm{keV}$ band. The luminosities are calculated from the observed-frame NUSTAR fluxes, assuming an effective photon index of $\Gamma_{\text {eff }}=1.8$ (as detailed in Section 2.4). The NUSTAR serendipitous survey covers a large range in $10-40 \mathrm{keV}$ luminosity; the large majority $(99.6 \% ; 238 / 239)$ of the unassociated sources lie in the range of $L_{10-40 \mathrm{keV}} \approx$ $10^{42}$ to $10^{46} \mathrm{erg} \mathrm{s}^{-1}$. The median luminosity of $1.2 \times$ $10^{44} \mathrm{erg} \mathrm{s}^{-1}$ is just above the "X-ray quasar" threshold. ${ }^{38}$ There is a single outlying source at very low luminosity and redshift, NuSTAR J115851+4243.2 (hereafter J1158; NLAGN, $z=$ $0.0023, L_{10-40 \mathrm{kev}}=1.0 \times 10^{39} \mathrm{erg} \mathrm{s}^{-1}$ ), hosted by the galaxy

\footnotetext{
38 A threshold of $10^{44} \mathrm{erg} \mathrm{s}^{-1}$ is often adopted to define "X-ray quasars," since this roughly agrees with the classical optical quasar definition $\left(M_{B} \leqslant-23\right.$ Schmidt \& Green 1983) and the $L_{\mathrm{X}}$,* value for unobscured AGNs (e.g., Hasinger et al. 2005).
} 


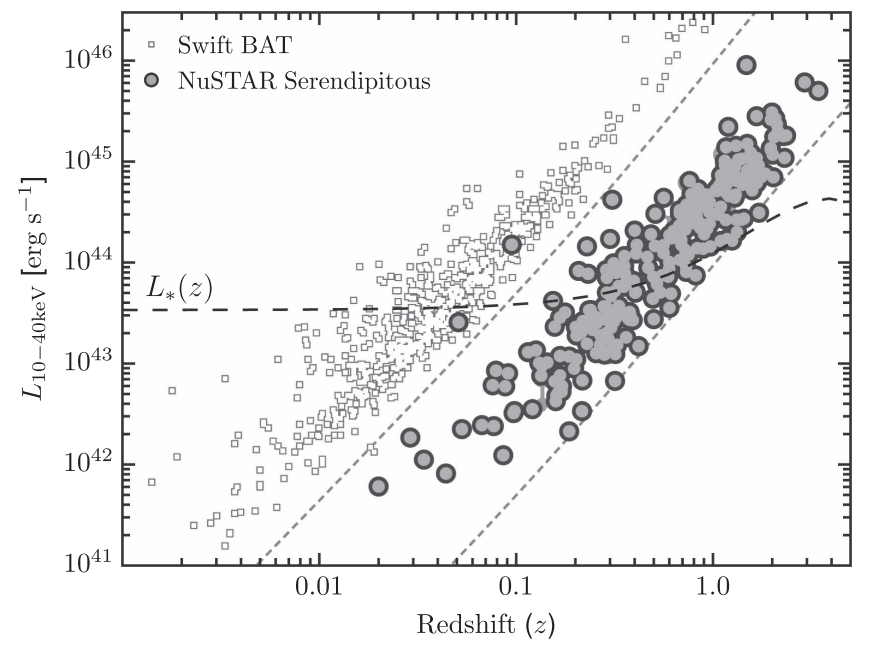

Figure 12. Rest-frame $10-40 \mathrm{keV}$ luminosity $\left(L_{10-40 \mathrm{keV}}\right)$ vs. redshift. We compare the NUSTAR serendipitous survey sample (circles) with the Swift BAT 70-month all-sky survey catalog (squares; Baumgartner et al. 2013; blazar and BL Lac types have been excluded). $L_{10-40 \mathrm{keV}}$ values for the Swift BAT sample are calculated from the $14-195 \mathrm{keV}$ values, assuming $\Gamma_{\text {eff }}=2.0$ for the $K$ correction factor (consistent with the median spectral slope for the Swift BAT sources shown). The gray short-dashed lines highlight an observed-frame X-ray flux range spanning two orders of magnitude, from $2 \times 10^{-14}$ to $2 \times 10^{-12} \mathrm{erg} \mathrm{s}^{-1} \mathrm{~cm}^{-2}$. The black long-dashed line shows the evolution of the knee of the X-ray luminosity function $\left(L_{*}\right)$ with redshift, as measured by Aird et al. (2015b). The NUSTAR serendipitous survey probes below $L_{*}$ at $z \lesssim 1$

IC 750. In this case, the SDSS optical spectrum shows an NLAGN superimposed over the galaxy spectrum. The source is discussed in detail in a work focusing on the NuSTAR-selected AGNs with dwarf galaxy hosts (Chen et al. 2017). At the other extreme end in luminosity is NuSTAR J052531-4557.8 (hereafter J0525; BLAGN, $z=1.479, L_{10-40 \mathrm{keV}}=9.0 \times$ $10^{45} \mathrm{erg} \mathrm{s}^{-1}$ ), also referred to as PKS 0524-460 in the literature. $^{39} \mathrm{~J} 0525$ has an effective NuSTAR photon index of $\Gamma_{\text {eff }}=1.9_{-0.2}^{+0.3}$ and a Swift XRT spectrum that is consistent with zero X-ray absorption. The optical spectrum of Stickel et al. (1993) shows a broad-line quasar with strong He II, C III], and $\mathrm{Mg}$ II emission lines. The source is also radio-bright (e.g., $f_{1.4 \mathrm{GHz}}=1.7 \mathrm{Jy}$; Tingay et al. 2003) and has been classified as a blazar in the literature (e.g., Massaro et al. 2009).

The most distant source detected is an optically unobscured quasar, NuSTAR J232728+0849.3 (hereafter J2327; BLAGN, $z=3.430, L_{10-40 \mathrm{keV}}=5.0 \times 10^{45} \mathrm{erg} \mathrm{s}^{-1}$ ), which represents the highest-redshift AGN identified in the NuSTAR survey program to date. Our Keck optical spectrum for J2327 shows a quasar spectrum with strong $\mathrm{Ly} \alpha, \mathrm{C}$ IV, and $\mathrm{C}$ III] emission lines and a well-detected Ly $\alpha$ forest. The source is consistent with having an observed X-ray spectral slope of $\Gamma_{\text {eff }}=2$ for both the NUSTAR spectrum and the XMM-Newton counterpart spectrum, and is thus in agreement with being unobscured at X-ray energies. The most distant optically obscured quasar detected is NuSTAR J125657+5644.6 (hereafter J1256; NLAGN $, \quad z=2.073, \quad L_{10-40 \mathrm{keV}}=2.7 \times 10^{45} \mathrm{erg} \mathrm{s}^{-1}$ ). Our Keck optical spectrum for J1256 reveals strong narrow Ly $\alpha$,

\footnotetext{
39 We note that J0525 appears in the Swift BAT all-sky catalog of Baumgartner et al. (2013) as a counterpart to the source SWIFTJ0525.3-4600. However, this appears to be a mismatch: an examination of the Swift BAT maps (following the procedures in Koss et al. 2013) and the NUSTAR data shows that J0525 is undetected by Swift BAT, and a nearby AGN in a foreground low-redshift galaxy ESO 253-G003 $(z=0.042)$ instead dominates the SWIFTJ0525.3-4600 counts.
}

C IV, He II, and C III] emission lines. Analyzing the NuSTAR spectrum in combination with a deep archival Chandra spectrum $(\approx 360 \mathrm{ks}$ of exposure in total), we measure a moderately large line-of-sight column density of $N_{\mathrm{H}}=$ $(1.3 \pm 0.4) \times 10^{23} \mathrm{~cm}^{-2}$. This distant quasar is thus obscured in both the optical and X-ray regimes.

In Figure 12 we compare with the 70-month Swift BAT allsky survey (Baumgartner et al. 2013). The two surveys are highly complementary; the Swift BAT all-sky survey provides a statistical hard-X-ray-selected sample of AGNs in the nearby universe (primarily $z<0.1$ ), while the NuSTAR serendipitous survey provides an equivalent sample (with comparable source statistics) for the distant universe. We compare with the redshift evolution of the knee of the X-ray luminosity function $\left(L_{\star}\right)$, as determined by Aird et al. (2015b). The Swift BAT all-sky survey samples the population below $L_{\star}$ for redshifts up to $z \approx 0.05$, while the NuSTAR serendipitous survey can achieve this up to $z \approx 1$. There is almost no overlap between the two surveys, which sample different regions of the parameter space. However, there are two NuSTAR sources, outlying in Figure 12, which have very high fluxes approaching the detection threshold of Swift BAT: NuSTAR J043727$4711.5 \quad\left(z=0.051, \quad L_{10-40 \mathrm{keV}}=2.6 \times 10^{43} \mathrm{erg} \mathrm{s}^{-1}\right) \quad$ and NuSTAR J075800+3920.4 $\left(z=0.095, L_{10-40 \mathrm{keV}}=1.5 \times\right.$ $10^{44} \mathrm{erg} \mathrm{s}^{-1}$ ). Both are BLAGNs (based on our Keck and NTT spectra) and are unobscured at X-ray energies $\left(\Gamma_{\text {eff }} \approx 1.9\right)$. The former is detected in the 70-month Swift BAT catalog of Baumgartner et al. (2013), and the latter is only detected with Swift BAT at the $\approx 2 \sigma$ level, based on the direct examination of the 104-month BAT maps (following the procedures in Koss et al. 2013).

In Figure 13 we compare the luminosity-redshift source distribution with other NuSTAR extragalactic survey samples: the NuSTAR-ECDFS survey (M15) and the NuSTAR-COSMOS survey (C15). Rest-frame luminosities are shown for the standard three NUSTAR bands $(3-8 \mathrm{keV}, 3-24 \mathrm{keV}$, and $8-24 \mathrm{keV})$. The serendipitous survey fills out the broadest range of luminosities and redshifts, due to the nature of the coverage (a relatively large total area, but with deep subareas that push to faint flux limits).

\subsection{Optical Properties}

\subsubsection{The X-Ray-Optical Flux Plane}

The X-ray-optical flux plane is a classic diagnostic diagram for sources detected in X-ray surveys (e.g., Maccacaro et al. 1988). This plane has recently been explored for the NuSTAR-COSMOS sample, using the $i$ band (C15). Here we investigate the plane using the optical $R$ band for the NuSTAR serendipitous survey, which provides a relatively large hard-Xray-selected sample spanning a comparatively wide flux range. The X-ray-to- $R$-band flux ratio $\left(f_{\mathrm{X}} / f_{\text {opt }}\right)$ diagnostic has been widely applied in past Chandra and XMM-Newton surveys of well-known blank fields (e.g., Hornschemeier et al. 2001; Barger et al. 2003; Fiore et al. 2003; Xue et al. 2011). Figure 14 shows the optical $R$-band magnitude $(R)$ against X-ray flux $\left(f_{\mathrm{X}}\right)$ for the $N U S T A R$ serendipitous survey sources that are detected in the hard band (8-24 keV) and full band (3-24 keV). We exclude $|b|<10^{\circ}$ and $z=0$ sources, thus minimizing contamination from Galactic sources. We subdivide the NuSTAR sample according to X-ray luminosity and optical spectroscopic classification: objects with successful identifications as either 


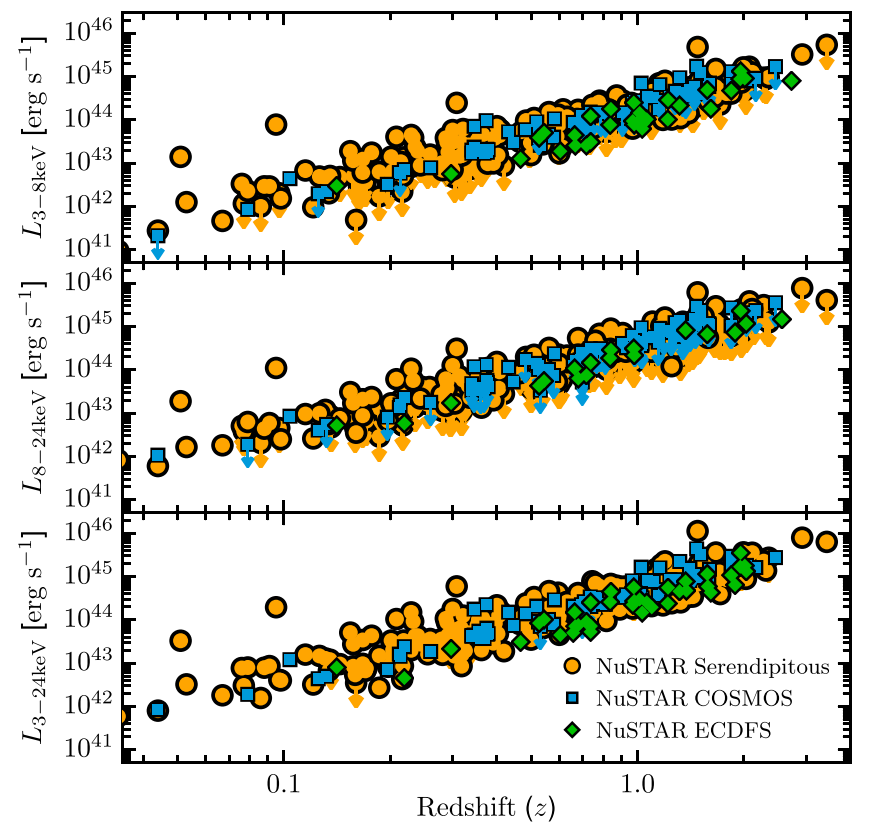

Figure 13. Luminosity vs. redshift for the three NuSTAR energy bands: 3-8 $\mathrm{keV}$ (top), 8-24 keV (middle), and 3-24 keV (bottom). We compare the NUSTAR serendipitous survey sample (orange circles) with the blank-field NUSTAR surveys of COSMOS (blue squares; C15) and ECDFS (green diamonds; M15).

NLAGNs or BLAGNs; objects with redshift constraints, but no classification; and objects with no redshift constraint or classification. For $R>20$, the sources shown with lower limits in $R$ generally correspond to a nondetection in the optical coverage, within the X-ray positional error circle. For sources where it is not possible to obtain an $R$-band constraint (e.g., due to contamination from a nearby bright star), we plot lower limits at the lower end of the $y$-axis.

We compare with the range of X-ray-to-optical flux ratios typically observed for AGNs identified in soft X-ray surveys, $-1<\log \left(f_{\mathrm{X}} / f_{\text {opt }}\right)<1$ (e.g., Schmidt et al. 1998; Akiyama et al. 2000; Lehmann et al. 2001). To illustrate constant X-ray-to-optical flux ratios, we adopt the relation of McHardy et al. (2003) and correct to the NUSTAR energy bands assuming $\Gamma_{\text {eff }}=1.8$. The large majority of sources lie at $\log \left(f_{\mathrm{X}} / f_{\text {opt }}\right)>-1$, in agreement with them being AGNs. At least $\approx 25 \%$ of the hard-band $(8-24 \mathrm{keV})$ selected sources lie at $\log \left(f_{\mathrm{X}} / f_{\text {opt }}\right)>1$, in agreement with the findings for the lowerenergy-selected X-ray sources detected in the Chandra and XMM-Newton surveys (e.g., Comastri et al. 2002; Fiore et al. 2003; Brandt \& Hasinger 2005). Such high $f_{\mathrm{X}} / f_{\text {opt }}$ values are interpreted as being driven by a combination of relatively high redshifts and obscuration (e.g., Alexander et al. 2001; Hornschemeier et al. 2001; Del Moro et al. 2008).

To demonstrate the dependence on X-ray luminosity and on spectral type, Figure 15 shows median $f_{\mathrm{X}} / f_{\mathrm{opt}}$ values for bins of X-ray luminosity, and for the NLAGN and BLAGN subsamples separately. The low-, intermediate-, and highluminosity bins correspond to $\log \left(L_{\mathrm{X}} / \mathrm{erg} \mathrm{s}^{-1}\right)<43,43<$ $\log \left(L_{\mathrm{X}} / \mathrm{erg} \mathrm{s}^{-1}\right)<44$, and $\log \left(L_{\mathrm{X}} / \mathrm{erg} \mathrm{s}^{-1}\right)>44$, respectively. The observed dependence on luminosity and on spectral type is consistent between the hard-band- and the full-bandselected samples (left and right panels of Figure 15, respectively). Overall, $f_{\mathrm{X}} / f_{\mathrm{opt}}$ increases with $\mathrm{X}$-ray luminosity. The

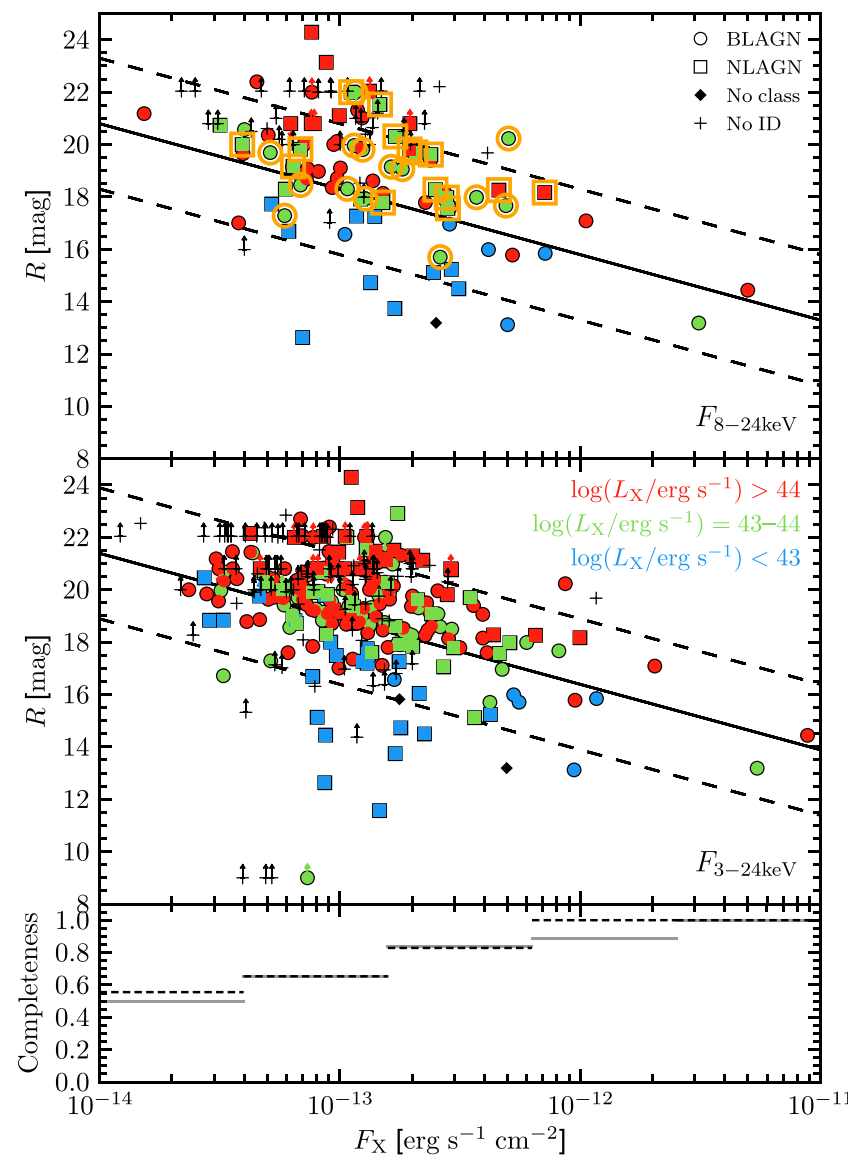

Figure 14. $R$-band optical magnitude $(R)$ vs. X-ray flux $\left(f_{\mathrm{X}}\right)$ for the hard-band (8-24 keV; top panel) and full-band (3-24 keV; middle panel) selected NuSTAR serendipitous survey samples. The blue, green, and red colors highlight three X-ray luminosity ranges, from low to high luminosity, respectively. The solid and dashed lines indicate constant X-ray-to-optical flux ratios of $\log \left(f_{\mathrm{X}} / f_{\text {opt }}\right)=0$ and \pm 1 , respectively. The hard-band subsample for which we calculate a reliable type 2 fraction (see Section 4.2.2) measurement is highlighted with orange outlines. In the bottom panel we show the optical spectroscopic completeness of both the 8-24 keV (dashed lines) and 3-24 keV (solid lines) samples as a function of $f_{\mathrm{X}}$, calculated as the number of sources with successful optical spectroscopic classifications (see Section 3.3.2) divided by the total number of sources in a given $f_{\mathrm{X}}$ bin.

increase between the low- and intermediate-luminosity bins is highly significant; for the hard-band-selected sample, the median $\log \left(f_{\mathrm{X}} / f_{\text {opt }}\right)$ value increases from $\approx-0.8$ to $\approx 0.5$. There is a marginally significant overall increase in $f_{\mathrm{X}} / f_{\mathrm{opt}}$ between the intermediate- and high-luminosity bins, which is driven by a significant increase in the $f_{\mathrm{X}} / f_{\mathrm{opt}}$ values of NLAGNs. A positive correlation between $f_{\mathrm{X}} / f_{\text {opt }}$ and $L_{\mathrm{X}}$ has previously been identified for Chandra and XMM-Newton samples of optically obscured AGNs selected at $<10 \mathrm{keV}$, over the same luminosity range (Fiore et al. 2003). Here we have demonstrated a strong positive correlation for high-energy ( $\gtrsim 10 \mathrm{keV}$ ) selected AGNs.

In general, the NLAGNs span a wider range in $f_{\mathrm{X}} / f_{\text {opt }}$ than the BLAGNs, which mostly lie within the range expected for BLAGNs based on soft X-ray surveys, $-1<\log \left(f_{\mathrm{X}} / f_{\text {opt }}\right)<1$. The most notable difference between the two classes is in the high-luminosity bin (which represents the "X-ray quasar" regime; $L_{\mathrm{X}}>10^{44} \mathrm{erg} \mathrm{s}^{-1}$ ), where the NLAGNs lie significantly higher than the BLAGNs, with a large fraction at $\log \left(f_{\mathrm{X}} / f_{\text {opt }}\right)>1$. This effect can be understood as a 


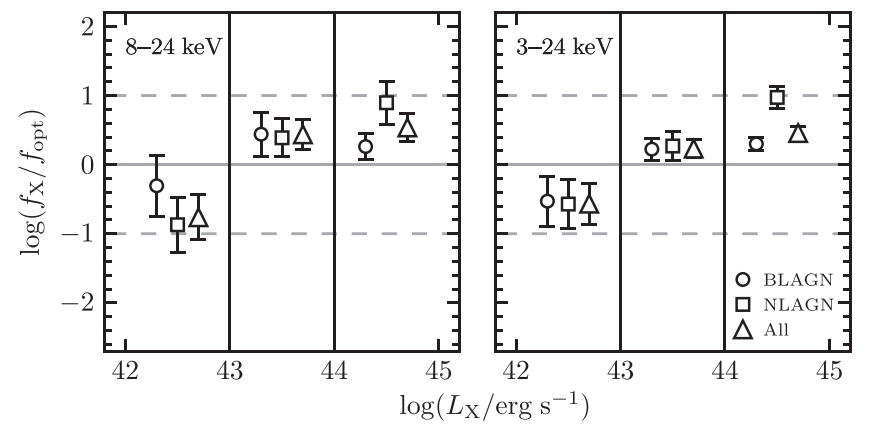

Figure 15. X-ray-to- $R$-band flux ratio $\left(f_{\mathrm{X}} / f_{\mathrm{opt}}\right)$, as a function of luminosity, for hard-band (8-24 keV) selected sources (left) and full-band (3-24 keV) selected sources (right). The luminosity bins follow those adopted in Figure 14. We show results for the overall spectroscopically identified population (triangles), BLAGN only (circles), and NLAGN only (squares). The solid and dashed horizontal gray lines indicate $\log \left(f_{\mathrm{X}} / f_{\text {opt }}\right)=0$ and \pm 1 , respectively. The horizontal offsets of the data points, within each luminosity bin, are arbitrary and for visualization purposes only.

consequence of extinction of the nuclear AGN emission. For the BLAGNs the nuclear optical-UV emission contributes strongly to the $R$-band flux, while for the NLAGNs the nuclear optical emission is strongly suppressed by intervening dust (the corresponding absorption by gas at X-ray energies is comparatively weak). The effect is augmented for the highluminosity bin, where the higher source redshifts $(\langle z\rangle \approx 0.9)$ result in the observed-frame optical band sampling a more heavily extinguished part of the AGN spectrum, while the observed-frame X-ray band samples a less absorbed part of the spectrum (e.g., Del Moro et al. 2008). The other main difference between the two classes is seen for the lowestluminosity bin, where, although the median flux ratios are consistent, the NLAGNs extend to lower values of $f_{\mathrm{X}} / f_{\text {opt }}$ than the BLAGNs, with a handful of the NLAGNs lying at $\log \left(f_{\mathrm{X}} / f_{\text {opt }}\right)<-1$.

\subsubsection{The Type 2 Fraction}

Here we investigate the relative numbers of the optically obscured (i.e., NLAGN) and optically unobscured (i.e., BLAGN) populations within the NUSTAR serendipitous survey sample. To provide meaningful constraints on the type 2 fraction (i.e., the observed number of NLAGNs divided by the total number of NLAGNs+BLAGNs), it is important to understand the sample completeness. We therefore investigate a specific subset of the overall sample for which completeness is well understood: hard-band $(8-24 \mathrm{keV})$ selected sources with $0.1<z<0.5,2 \times 10^{43}<L_{10-40 \mathrm{keV}}<2 \times 10^{44} \mathrm{erg} \mathrm{s}^{-1}$, and $|b|>10^{\circ}$ (highlighted with orange outlines in the top panel of Figure 14). The redshift limit ensures that the subsample has high spectroscopic completeness (i.e., the majority of sources have redshifts and classifications from optical spectroscopy; see below), the lower luminosity limit ensures "X-ray completeness" (i.e., the AGN population within this $L_{X}-z$ parameter space has fluxes that lie above the NuSTAR detection limit; e.g., see Figure 12), and the upper luminosity limit is applied to allow comparisons with luminosity-matched comparison samples (see below). The luminosity range samples around the knee of the X-ray luminosity function $\left(L_{*}\right)$ for these redshifts, $L_{10-40 \mathrm{keV}} \approx(4-7) \times$ $10^{43} \mathrm{erg} \mathrm{s}^{-1}$ (Aird et al. 2015b). In total, there are 30 spectroscopically identified sources (all NLAGNs or BLAGNs) within this subsample, which have a median redshift of $\langle z\rangle=0.3$. Accounting for sources that are not spectroscopically identified,

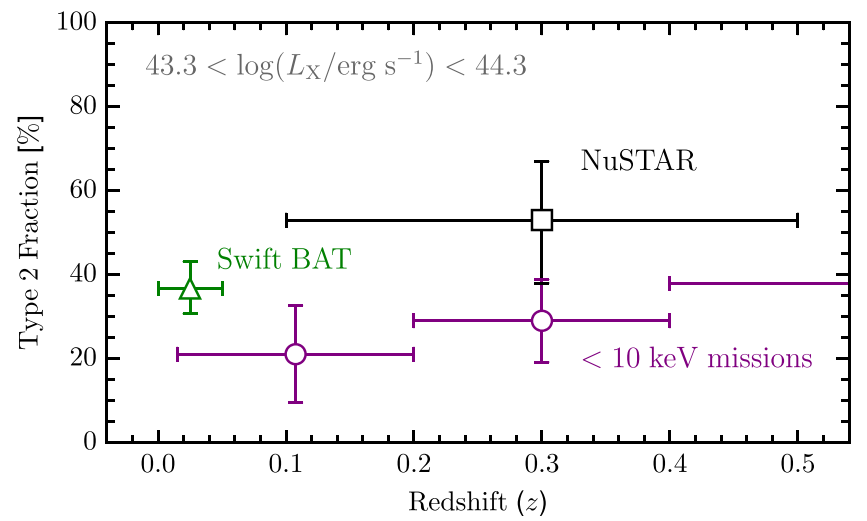

Figure 16. Observed type 2 fraction vs. redshift for various luminositymatched $\left(2 \times 10^{43} \mathrm{erg} \mathrm{s}^{-1}<L_{\mathrm{X}}<2 \times 10^{44} \mathrm{erg} \mathrm{s}^{-1}\right)$, X-ray-selected AGN samples: the black square shows a hard-band (8-24 keV) selected subset of the NuSTAR serendipitous survey sample with $0.1<z<0.5$, the green triangle shows a subset of the 70-month Swift BAT all-sky survey sample $(z<0.05$; Baumgartner et al. 2013), and the purple circles correspond to a $<10 \mathrm{keV}$ selected AGN sample, compiled from multiple X-ray surveys (including ASCA, Chandra, and XMM-Newton surveys; Hasinger 2008). The horizontal error bars show the redshift limits of each subsample.

we estimate an effective spectroscopic completeness of $97 \%$ $100 \%$ for this subsample (details are provided in Appendix A.4).

The observed type 2 fraction for the NuSTAR hard-bandselected subsample described above is $F_{\text {Type } 2}=53_{-15}^{+14} \%$ (binomial uncertainties). If we instead use the sources selected in the full band $(3-24 \mathrm{keV})$, a similar fraction is obtained $\left(F_{\text {Type } 2}=48 \pm 11 \%\right)$. In Figure 16 we compare with the type 2 fraction for nearby $(z<0.05)$ AGNs similarly selected at high X-ray energies $(>10 \mathrm{keV})$. To obtain this data point, we calculate the observed type 2 fraction for the 70-month Swift BAT all-sky survey. Importantly, we use a luminositymatched subsample of the Swift BAT survey $\left(2 \times 10^{43}<\right.$ $L_{10-40 \mathrm{keV}}<2 \times 10^{44} \mathrm{erg} \mathrm{s}^{-1}$, as for the NuSTAR subsample), since the type 2 fraction likely varies with luminosity. We apply a redshift cut of $z<0.05$ to ensure X-ray completeness (redshifts above this threshold push below the flux limit of Swift BAT for the adopted $L_{10-40 \mathrm{keV}}$ range; see Figure 12). For consistency with our approach for the NUSTAR sample, we class Swift BAT AGNs with intermediate types of 1.9 or below as BLAGNs, those with NL Sy1 type spectra as BLAGNs, and those with galaxy type optical spectra as NLAGNs. The observed type 2 fraction for this luminosity-matched Swift BAT sample at $z<0.05$ is $F_{\text {Type } 2}=37 \pm 6 \%$, slightly lower than our NuSTAR-measured type 2 fraction at $z \approx 0.3$, but consistent within the uncertainties. A caveat to this comparison is that the spectroscopic completeness of the Swift BAT subsample is unknown; overall there are $\approx 100$ sources in the Baumgartner et al. (2013) catalog that are consistent with being AGNs but lack an optical spectroscopic redshift and classification, some of which could potentially lie within the luminosity and redshift ranges adopted above. Making the extreme assumption that these $\approx 100$ sources all lie in the above luminosity and redshift ranges and are all NLAGNs, the maximum possible Swift BAT $F_{\text {Type } 2}$ value is $66 \%$ (which would still be in agreement with the NUSTAR-measured fraction). Depending on the full duration of the NUSTAR mission, the source numbers for the NUSTAR serendipitous survey may feasibly increase by a factor of two or more, which will reduce the uncertainties on the type 2 fraction. However, to determine reliably whether there is evolution in the type 2 fraction of high-energy-selected AGNs 
between $z<0.05$ and $z>0.1$, future studies should systematically apply the same optical spectroscopic classification methodologies to both samples. An early indication that the obscured fraction of AGNs might increase with redshift was given by La Franca et al. (2005), and this has been further quantified in subsequent works (e.g., Ballantyne et al. 2006; Treister \& Urry 2006; Hasinger 2008; Merloni et al. 2014). The slope of the increase with redshift is consistent with that found by Treister \& Urry (2006).

The type 2 fraction has been thoroughly investigated for the AGN population selected by lower-energy $(<10 \mathrm{keV})$ X-ray missions such as Chandra and XMM-Newton. Hasinger (2008) presented a relatively complete $2-10 \mathrm{keV}$ selected sample, compiled from a variety of surveys with $<10 \mathrm{keV}$ missions (also see Merloni et al. 2014, for a more recent study of XMMNewton-selected sources at $0.3<z<3.5)$. We consider the $0.2<z<0.4$ subset of the Hasinger (2008) sample, in order to match to our NuSTAR subsample in redshift as closely as possible, and we limit to the luminosity range explored above $\left(2 \times 10^{43}<L_{10-40 \mathrm{keV}}<2 \times 10^{44} \mathrm{erg} \mathrm{s}^{-1} ;\right.$ we assume a luminosity band correction of $L_{10-40 \mathrm{keV}} / L_{2-10 \mathrm{keV}}=1$ ). The type 2 fraction for this subset of the Hasinger (2008) sample is $F_{\text {Type 2 }}=29 \pm 10 \%$, which is lower than our NuSTARmeasured type 2 fraction (see Figure 16), but only at a significance level of $\approx 2 \sigma$. This could be explained as a result of the different selection functions of different X-ray missions, with the high-energy $(>8 \mathrm{keV}$ ) selection of $N u S T A R$ being less biased against obscured sources. Another factor to consider is the different classification methodologies applied. In addition to optical spectroscopic constraints, Hasinger (2008) use additional $\mathrm{X}$-ray hardness information to classify ambiguous sources as NLAGNs or BLAGNs. Hasinger (2008) do assess the extent to which the type 2 fraction measurements change if, instead, only the pure optical spectroscopic classification is adopted (i.e., a similar approach to our spectroscopic classification for the NuSTAR sources) and find that, for the redshift and luminosity ranges explored here, the type 2 fraction would be somewhat higher but unlikely to increase by more than a factor of $\approx 1.2$.

In Figure 16 we compare with additional luminositymatched subsamples for the adjacent redshift bins studied by Hasinger (2008). The high-energy-selected AGN samples (NuSTAR and Swift BAT) appear to lie systematically higher in type 2 fraction than the luminosity-matched lower-energy $(<10 \mathrm{keV})$ selected AGNs, for the redshift ranges covered. We note that the type 2 fraction constraints of Merloni et al. (2014) for $<10 \mathrm{keV}$ selected AGNs are broadly consistent with the values shown in Figure 16 (we primarily compare with the Hasinger [2008] sample since the source redshifts and luminosities sampled facilitate a direct comparison of results). The apparently small numbers of CT AGNs identified (e.g., see Figure 10) suggest that the offset in type 2 fraction is not primarily driven by the uncovering of a new CT population, but more likely by the selection functions of NuSTAR and Swift BAT being generally less biased against significantly obscured AGNs.

\subsection{Infrared Properties}

\subsubsection{WISE Colors}

Mid-infrared (MIR; $\gtrsim 5 \mu \mathrm{m}$ ) emission from AGNs is primary emission that has been reprocessed by circumnuclear dust and suffers little extinction relative to other (e.g., optical and soft
$\mathrm{X}$-ray) wavelengths. Color selections using the WISE telescope bands (e.g., Assef et al. 2010, 2013; Jarrett et al. 2011; Mateos et al. 2012, 2013; Stern et al. 2012) can separate bright AGNs from host-galaxy light (from stars and the interstellar medium) through the identification of a red MIR spectral slope and have thus become widely applied. These selections have the potential to identify large samples of AGNs with less bias against heavily obscured systems. However, their effectiveness worsens toward lower AGN luminosities, where identifying the AGN component of the MIR spectrum is more problematic. Here we investigate the MIR properties of our NuSTAR serendipitous survey sample and consider the results with respect to these AGN selection criteria.

Figure 17 shows a WISE color-color diagram (W1-W2 versus $W 2-W 3$ ) for the NUSTAR serendipitous survey subsamples that are selected (i.e., independently detected) in the hard band (8-24 keV; top panel) and full band (3-24 keV; bottom panel). In general, the sources that lie at higher (i.e., redder) $W 1-W 2$ values have stronger AGN contributions to their MIR SEDs. We exclude low Galactic latitude sources $\left(|b|<10^{\circ}\right)$ and sources that are spectroscopically confirmed as Galactic. In addition, we only consider sources with wellconstrained X-ray positions (i.e., with Chandra, Swift XRT, or $X M M-N e w t o n$ positions), and we limit the analysis to the fraction of these sources $(70 \%$ and $61 \%$ for the hard and full band, respectively) with significant detections in all three of the relevant, shorter-wavelength WISE bands ( $W 1, W 2$, and $W 3$, which are centered at $3.4,4.6$, and $12 \mu \mathrm{m}$, respectively). Figure 17 shows the sample subdivided according to X-ray luminosity and optical spectral classification. In Figure 18 we show the fraction ( $f_{\text {wedge }}$ hereafter) of sources that are selected as AGNs based on MIR colors alone, according to the selection "wedge" of Mateos et al. (2012), as a function of X-ray luminosity and optical classification.

For the NUSTAR AGNs selected in the full band (bottom panel of Figure 17 and right panel of Figure 18) the overall fraction of sources identified as AGNs in the MIR is $f_{\text {wedge }}=64.9_{-6.2}^{+5.7} \%$ (111/171). Considering sources with optical spectroscopic classifications, the fractions for the overall BLAGN and NLAGN samples are $f_{\text {wedge }}=77.6_{-7.2}^{+5.9} \% \quad(83 / 107)$ and $48.9_{-11.8}^{+11.9} \%$ (22/45), respectively. NLAGNs are therefore significantly less likely to be identified as AGNs based on MIR colors alone. This is largely driven by the lower luminosity, on average, of the NLAGNs (median of $4 \times 10^{43} \mathrm{erg} \mathrm{s}^{-1}$ ) compared to the BLAGNs (median of $3 \times 10^{44} \mathrm{erg} \mathrm{s}^{-1}$ ), in combination with the fact that $f_{\text {wedge }}$ decreases toward lower luminosities (see Figure 18). Matching the NLAGNs and BLAGNs in luminosity, we do not find statistically significant differences in $f_{\text {wedge }}$ between the two classes.

For the remainder of the members of our overall sample that lack optical spectroscopic classifications (gray crosses in Figure 17), the WISE colors are informative of their likely properties. A low fraction of these sources lie within the wedge, $f_{\text {wedge }}=27.8_{-13.5}^{+19.3}(5 / 18)$. This suggests that, statistically, the unidentified sources are likely to be less luminous AGNs. In combination with the poor success rate for optical spectroscopy of these sources, we expect that they are likely to be dominated by optically obscured, low-luminosity systems.

The results in Figure 18 show that MIR selections miss a significant fraction of the $N U S T A R$-selected AGN population, with the missed fraction increasing from $\approx 15 \%$ at high luminosities to $\approx 80 \%$ at the lower-luminosity end. The 


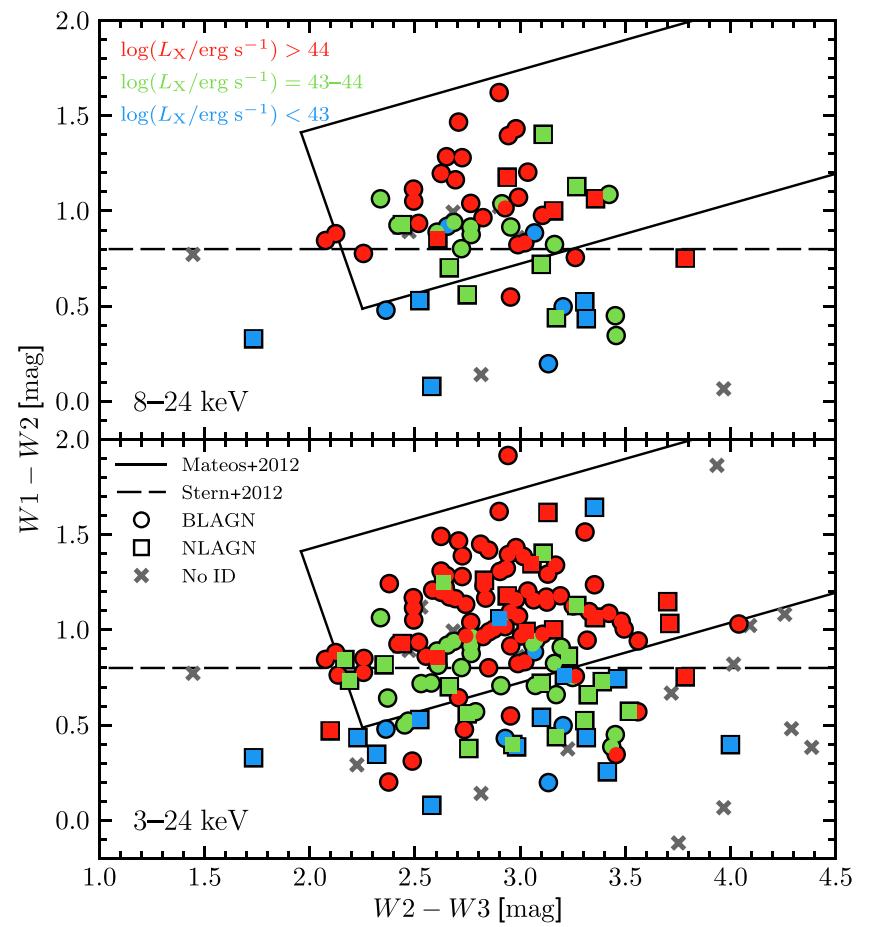

Figure 17. WISE color-color diagram of NUSTAR serendipitous survey AGNs as a function of X-ray luminosity $\left(L_{\mathrm{X}}\right)$ and source classification, for hard-band (8-24 keV) selected sources (top) and full-band (3-24 keV) selected sources (bottom). BLAGNs and NLAGNs are shown as circles and squares, respectively, while sources without a spectroscopic identification are shown as gray crosses. The blue, green, and red colors highlight three X-ray luminosity ranges, from low to high luminosity, respectively. The luminosities correspond to the selection bands used for this analysis (i.e., $L_{8-24} \mathrm{keV}$ and $L_{3-24 \mathrm{keV}}$ for the top and bottom panels, respectively). We compare with the AGN "wedge" of Mateos et al. (2012) and the AGN color cut of Stern et al. $(2012 ; W 1-W 2 \geqslant 0.8)$.

dependence of MIR selections on AGN luminosity has been identified for lower-energy X-ray-selected AGN samples (e.g., Cardamone et al. 2008; Eckart et al. 2010) and is likely primarily driven by a stronger contribution to the SED from the host galaxy for lower X-ray luminosities, which results in bluer MIR colors. The MIR AGN selection wedge of Mateos et al. (2012) was defined using the Bright Ultrahard XMM-Newton Survey (BUXS) sample, selected at $4.5-10 \mathrm{keV}$, for comparable numbers of spectroscopically identified AGNs (114 BLAGNs and 81 NLAGNs) as the full-band-selected NuSTAR serendipitous survey sources incorporated here (107 BLAGNs and 45 NLAGNs), and for a similar redshift and luminosity distribution. For the NLAGNs, our results for $f_{\text {wedge }}$ as a function of X-ray luminosity and optical classification are consistent (given the uncertainties) with those found for the BUXS sample. Our BLAGNs have marginally lower $f_{\text {wedge }}$ values than the BUXS BLAGNs. For instance, Mateos et al. (2012) find that the MIR selection is essentially complete for BLAGNs at $L_{\mathrm{X}}>10^{43} \mathrm{erg} \mathrm{s}^{-1}$ (e.g., $f_{\text {wedge }}=100_{-6.6} \%$ and $96.1_{-6.3}^{+3.0} \%$ for $L_{2-10 \mathrm{keV}}=10^{43}-10^{44}$ and $10^{44}-10^{45} \mathrm{erg} \mathrm{s}^{-1}$, respectively), while even at the highest luminosities $\left(L_{3-24 \mathrm{keV}}>10^{44} \mathrm{erg} \mathrm{s}^{-1}\right)$ we find $f_{\text {wedge }}=84.2_{-8.0}^{+5.7} \%$.

It is notable that the MIR selection fails for 12 (i.e., 15.8\%) of the high-luminosity NuSTAR-selected BLAGNs, since MIR selections are typically expected to be close to complete for high-luminosity, unobscured sources. To assess why these sources in particular are not MIR selected, we compare their

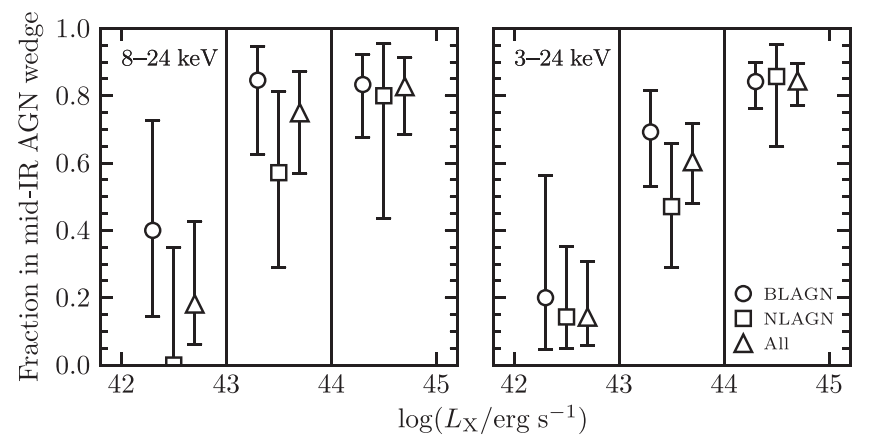

Figure 18. Fraction of extragalactic NUSTAR serendipitous survey sources that are selected as AGNs based on MIR colors alone (i.e., they lie in the WISE color wedge of Mateos et al. 2012), as a function of luminosity, for hard-band (8-24 keV) selected sources (left) and full-band (3-24 keV) selected sources (right). The luminosity bins follow those adopted in Figure 17. We show results for the overall spectroscopically identified population (triangles), BLAGN only (circles), and NLAGN only (squares). The error bars show binomial uncertainties. The horizontal offsets of the data points, within each luminosity bin, are arbitrary and for visualization purposes only.

source properties (e.g., NUSTAR detection significance, redshift, 10-40 keV luminosity, 2-10 keV luminosity, brightness, optical spectra, Galactic latitude) with the 64 (i.e., the $84.2 \%$ ) high-luminosity BLAGNs that are MIR selected. There are no clear statistically significant differences, with a possible exception: the optical $R$-band magnitude distributions of the two subsets are different at a moderate significance level (KStest $p$-value of $p=0.037$ ), with the 12 MIR-unselected sources skewed to fainter $R$ values (median of $\langle R\rangle=19.9$ ) than their MIR-selected counterparts $(\langle R\rangle=19.4)$. This result increases in significance (to $p=0.0075$ ) if we limit the comparison to the eight (out of 12) MIR-unselected sources that are additionally missed by the Stern et al. (2012) $W 1-W 2$ color AGN selection. Comparing the distribution of $f_{\mathrm{X}} / f_{\mathrm{opt}}$ versus $W 1-W 2$ for these eight sources with the overall serendipitous sample (see Figure 19), they overlap with lower-luminosity AGNs where we expect that the relatively blue $W 1-W 2$ colors are driven by a stronger (relative) contribution to the MIR SED from the host galaxy. The latter could also be true for the eight MIR-unselected high- $L_{X}$ BLAGNs if their MIR AGN luminosities are relatively low compared to the 64 MIR-selected counterparts (which are matched in X-ray luminosity). Estimating the rest-frame $6 \mu \mathrm{m}$ luminosities $\left(L_{6 \mu \mathrm{m}}\right)$ by interpolating between the relevant observed-frame WISE band magnitudes, ${ }^{40}$ we find that the eight MIR-unselected BLAGNs have a different $L_{6 \mu \mathrm{m}}$ distribution from the MIR-selected counterparts $(p=0.046)$ and are indeed skewed to lower MIR luminosities $\left(\left\langle L_{6 \mu \mathrm{m}}\right\rangle=3.4 \times 10^{44} \mathrm{erg} \mathrm{s}^{-1}\right)$ than the MIRselected sources $\left(\left\langle L_{6 \mu \mathrm{m}}\right\rangle=1.3 \times 10^{45} \mathrm{erg} \mathrm{s}^{-1}\right)$. In summary, the incompleteness of MIR selections for unobscured high$L_{\mathrm{X}} \mathrm{AGN}$ appears to be related to scatter in the intrinsic AGN properties. The luminous MIR-unselected sources could potentially represent AGNs that are lacking in hot-dust emission (i.e., "hot-dust-poor" AGNs; e.g., Hao et al. 2010), although the inferred hot-dust-poor fraction $(\sim 10 \%-15 \%)$ would be unexpectedly high compared to that observed for optically selected quasars ( 1\%; Jun \& Im 2013).

\footnotetext{
${ }^{40}$ From the WISE all-sky survey catalog, there are no indications of bad photometry (e.g., due to blending, contamination, or confusion) for these eight sources.
} 


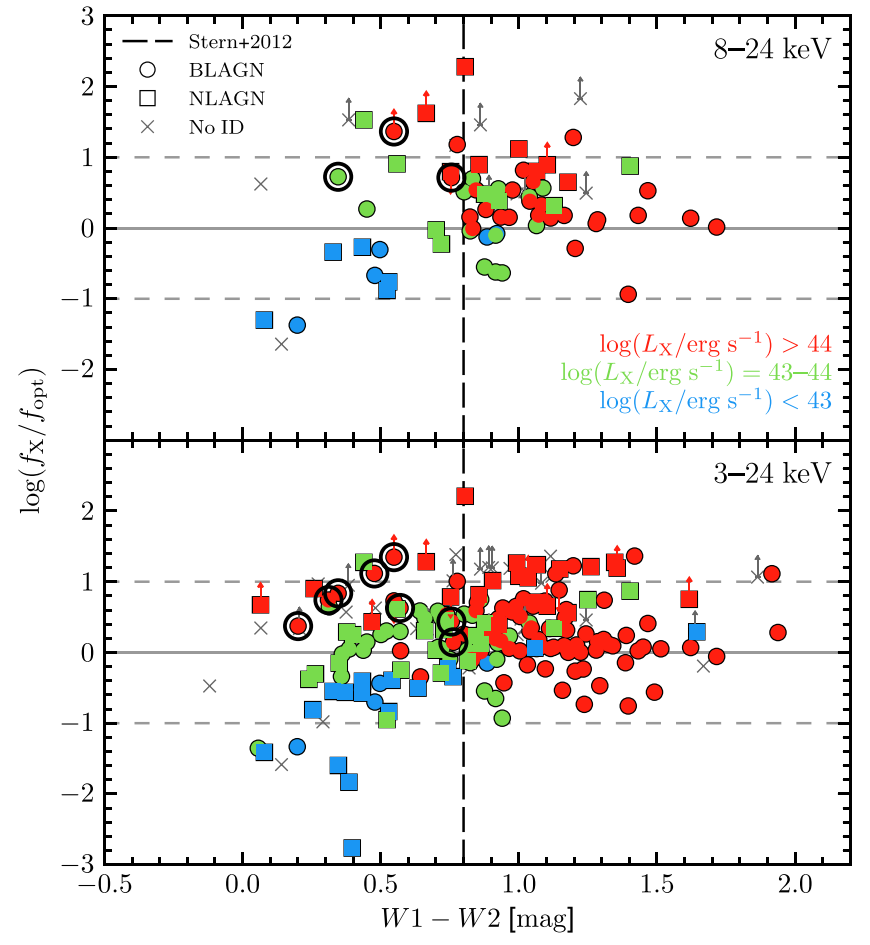

Figure 19. X-ray-to- $R$-band flux ratio $\left(f_{\mathrm{X}} / f_{\text {opt }}\right)$ vs. the WISE $W 1-W 2$ color, for hard-band (8-24 keV) selected sources (top) and full-band (3-24 keV) selected sources (bottom). The luminosity bins and the marker labeling follow those adopted in Figures 14 and 17. Eight high-luminosity BLAGNs that are not selected as AGNs in the MIR (see Section 4.3.1) are highlighted with large black circles.

For the NUSTAR serendipitous survey sources selected in the hard band (top panel of Figure 17 and left panel of Figure 18), for which NUSTAR is uniquely sensitive, the results are consistent with those for the full-band sample, but with greater uncertainties due to the smaller source numbers. For instance, $f_{\text {wedge }}=67.6_{-9.8}^{+8.5} \%(46 / 68)$ for the overall hard-band-selected sample. We conclude that, while there are some small differences, the MIR color distribution of the NUSTAR serendipitous survey sample is largely consistent with that expected based on the results for lower-energy $(<10 \mathrm{keV})$ selected AGNs.

\subsubsection{X-Ray-MIR Luminosity Plane}

There is a remarkably tight correlation between the X-ray luminosities and the MIR luminosities of unobscured AGNs, with both providing estimates of the intrinsic AGN power (e.g., Lutz et al. 2004; Fiore et al. 2009; Gandhi et al. 2009; Lanzuisi et al. 2009; Ichikawa et al. 2012; Matsuta et al. 2012; Asmus et al. 2015; Mateos et al. 2015; Stern 2015). Low X-ray-to-MIR luminosity ratios are interpreted as being due to either X-ray absorption or intrinsic X-ray weakness.

In Figure 20 we show the observed (i.e., uncorrected for absorption) rest-frame X-ray luminosities $\left(L_{\mathrm{X}}^{\mathrm{obs}}\right)$ versus the restframe $6 \mu \mathrm{m}$ luminosities ( $L_{6 \mu \mathrm{m}}$, in $\nu L_{\nu}$ units) for NuSTAR serendipitous survey sources. We only include sources that are AGN dominated at MIR wavelengths according to the WISE colors (based on either of the criteria in Section 4.3.1), and thus where we believe the rest-frame $6 \mu \mathrm{m}$ flux to be dominated by the AGN rather than host-galaxy light. Additionally, we require the sources to be detected in the two observed-frame WISE bands that are interpolated between to estimate $L_{6 \mu}$ m (e.g., $W 2$

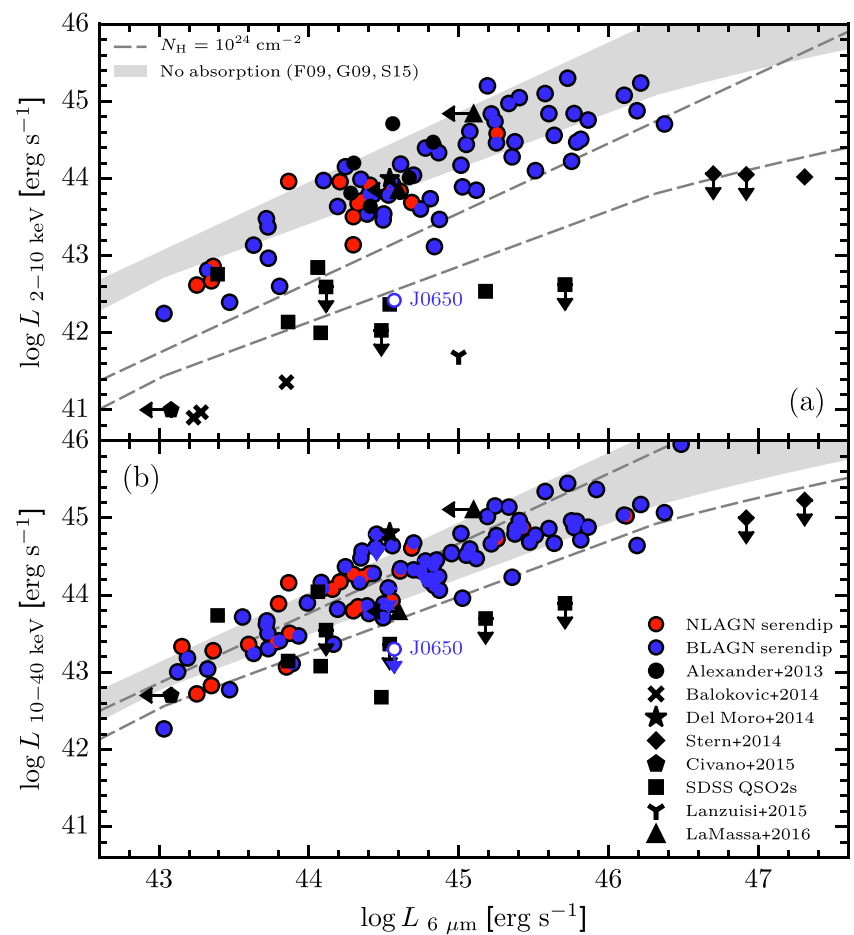

Figure 20. Observed (i.e., uncorrected for absorption) rest-frame (a) $2-10 \mathrm{keV}$ $\left(L_{2}-10 \mathrm{keV}\right)$ and (b) $10-40 \mathrm{keV}$ X-ray luminosity $\left(L_{10-40 \mathrm{keV}}\right)$ vs. rest-frame $6 \mu \mathrm{m}$ luminosity $\left(L_{6 \mu \mathrm{m}}\right.$, in $\nu L_{\nu}$ units). Filled circles show the NuSTAR serendipitous survey sources. We only show sources that have detections in the WISE bands necessary to estimate $L_{6} \mu \mathrm{m}$ (through interpolation), and which are AGN dominated at MIR wavelengths according to their WISE colors (based on satisfying either the Mateos et al. (2012) or Stern et al. (2012) criteria), and thus where we believe $L_{6 \mu \mathrm{m}}$ to have minimal contamination from the host galaxy. The apparently X-ray weak source J0650 (see Section 4.3.2 and Appendix A.5) is shown as an open circle and labeled. We compare with other samples: NUSTAR-observed SDSS-selected heavily obscured type 2 quasars (squares; $z=0.05-0.49$; Gandhi et al. 2014; Lansbury et al. 2014, 2015), three CT Seyfert 2 AGNs from the NUSTAR snapshot survey (crosses; $z \approx 0.01$; Baloković et al. 2014), luminous and heavily obscured WISE-selected AGNs targeted with $N u S T A R$ (diamonds; $z \approx 2$; Stern et al. 2014), a heavily obscured quasar identified in the NuSTAR-ECDFS survey (star; $z \approx 2$; Del Moro et al. 2014); a CT AGN identified in the NuSTAR-COSMOS survey (pentagon; ID 330; $z=0.044 ; \mathrm{C} 15)$, a candidate heavily CT AGN identified in the COSMOS field (triangle; $z=0.35$; Lanzuisi et al. 2015), and NuSTARobserved FIRST-2MASS red quasars (triangle; $z=0.14-0.41$; LaMassa et al. 2016). All of the data are compared with the luminosity ratios expected in the case of zero line-of-sight absorption (gray region). This region shows the range of intrinsic luminosity ratios predicted by three different intrinsic relations in the literature: Gandhi et al. (2009), Fiore et al. (2009), and Stern (2015). The dashed lines illustrate the observed X-ray luminosity suppression expected if the zero absorption region is absorbed by gas with a column density of $N_{\mathrm{H}}=10^{24} \mathrm{~cm}^{-2}$.

and $W 3$ for $z<1)$. For the high-energy $(10-40 \mathrm{keV})$ rest-frame $\mathrm{X}$-ray band (bottom panel of Figure 20), the X-ray luminosities are from NUSTAR photometry (as described in Section 2.4). For the low-energy $(2-10 \mathrm{keV})$ rest-frame X-ray band (top panel of Figure 20), the X-ray luminosities are estimated from CSC or 3XMM counterpart fluxes (for the top panel, we only show sources with counterparts in these catalogs). We compare with other NUSTAR-observed samples, including a number of heavily obscured AGNs. To demonstrate the approximate Xray-to-MIR luminosity ratios expected in the cases of zero absorption and high absorption, we show the intrinsic X-rayMIR relation (as measured by multiple studies; Fiore et al. 2009; Gandhi et al. 2009; Stern 2015) and the same relation after absorption by $N_{\mathrm{H}}=10^{24} \mathrm{~cm}^{-2}$ gas, respectively. 
At $10-40 \mathrm{keV}$, the serendipitous survey sources are generally consistent with both the intrinsic and the highly absorbed X-ray-MIR relations (which are close together for these energies). The most outlying source, J0650 (highlighted in Figure 20), has a very low upper limit in X-ray-to-MIR luminosity ratio. Notably, for this source the Keck optical spectroscopy reveals a narrow-line Seyfert 1 (NL Sy1) spectrum, and we measure a very steep $0.5-10 \mathrm{keV}$ X-ray spectrum $\left(\Gamma_{\text {eff }}=3.1\right)$. Given these properties, we interpret the low X-ray-to-MIR ratio as likely being driven by intrinsic X-ray weakness (in combination with the steep X-ray spectrum), rather than being driven by extreme absorption levels. Intrinsic X-ray weakness has previously been identified for objects in the NL Sy1 class (e.g., Leighly et al. 2007a, 2007b; Miniutti et al. 2012). A detailed discussion of J0650 is provided in Appendix A.5.

At $2-10 \mathrm{keV}$, the sample shows evidence for significant downward deviations from the intrinsic relations, although there is little overlap with the known heavily absorbed and CT sources that have been observed in targeted NuSTAR programs. We note, however, that this analysis is currently limited to a specific subset of the serendipitous survey (i.e., sources that are AGN dominated at MIR wavelengths and that are detected in the relevant WISE bands). Future SED modeling of the broader spectroscopically identified sample would allow reliable $L_{6 \mu \mathrm{m}}$ measurements (disentangling AGN and host-galaxy MIR emission) for a more complete subset of the serendipitous survey sample.

\section{Summary}

The high sensitivity of $N U S T A R$ at $\gtrsim 10 \mathrm{keV}$ has provided access to large samples of high-energy X-ray-emitting AGNs in the distant universe, whereas previous observatories were largely restricted to the local universe $(z \lesssim 0.1)$. In this paper we have presented the first full catalog for the NuSTAR serendipitous survey, the largest survey undertaken with NuSTAR, which incorporates data from the first 40 months of telescope operation. The data include 331 unique fields, with a total areal coverage of $13 \mathrm{deg}^{2}$ and a cumulative exposure time of $\approx 20$ Ms. We have characterized the NuSTAR-detected AGNs in terms of their X-ray, optical, and IR properties. Below we summarize the main results:

1. Overall, we detect 497 sources that are significant postdeblending (i.e., after accounting for contamination of the photon counts from nearby sources). Of these, 163 are independently detected in the hard (8-24 keV) energy band; see Section 2.5.

2. The median vignetting-corrected exposure time per source (for the combined FPMA+FPMB data) is $\left\langle t_{\text {net }}\right\rangle=60 \mathrm{ks}$, and the maximum is $1500 \mathrm{ks}$. The $\mathrm{X}$-ray fluxes span from $f_{3-24 \mathrm{keV}} \approx 10^{-14}$ to $10^{-11} \mathrm{erg} \mathrm{s}^{-1} \mathrm{~cm}^{-2}$, with a median value of $\left\langle f_{3-24 \mathrm{keV}}\right\rangle=1.1 \times 10^{-13} \mathrm{erg} \mathrm{s}^{-1} \mathrm{~cm}^{-2}$; see Section 4.1.1. The survey reaches flux depths similar to the NuSTAR surveys in well-studied fields (COSMOS, ECDFS, EGS, GOODS-N, and UDS) over comparable areas (see Section 2.3) and is $\approx 2$ orders of magnitude fainter than the Swift BAT surveys; e.g., see Section 4.1.3.

3 . There is a large range in the observed band ratios of AGNs at 3-24 keV, which imply a range of effective photon indices going from very soft $\left(\Gamma_{\text {eff }} \approx 3\right)$ to very hard $\left(\Gamma_{\mathrm{eff}} \approx 0\right)$; see Section 4.1.2. We find no evidence for an anticorrelation between band ratio and count rate, as has previously been found for lower-energy X-ray bands; see Section 4.1.2.

4. A large fraction $79 \%(395 / 497)$ of the sources have soft $(<10 \mathrm{keV}) \mathrm{X}$-ray counterparts detected in surveys or archival data from XMM-Newton, Chandra, and Swift XRT. The NuSTAR fluxes and the soft X-ray counterpart fluxes show good agreement for the $3-8 \mathrm{keV}$ energy band, and the maximum identified variation in AGN flux between the soft $\mathrm{X}$-ray and NUSTAR observations is a factor of $\approx 5$; see Section 3.1. The higher positional accuracies of the soft X-ray observatories relative to $N u S T A R$ allow us to reliably match to optical and IR counterparts; see Section 3.2.

5. Optical spectroscopic identifications (i.e., redshift measurements and source classifications) have been successfully obtained for 276 sources. For the large majority of the sample (222 sources) this was achieved through our extensive campaign of ground-based spectroscopic follow-up, using a range of observatories at multiple geographic latitudes; see Section 3.3. Sixteen sources are spectroscopically confirmed as Galactic. Of the 260 extragalactic sources (AGNs), 162 (62.3\%) are classified as BLAGNs, $97(37.3 \%)$ are NLAGNs, and $1(0.4 \%)$ is a BL Lac; see Section 3.3.2. While similar numbers of NLAGNs and BLAGNs are identified at lower redshifts $(z \lesssim 1)$, there is a bias toward detections of BLAGNs at higher redshifts; this bias has been well established for other X-ray missions (e.g., Chandra and XMM-Newton); see Section 4.1.3.

6. The serendipitous survey AGNs have redshifts covering a wide range, from $z=0.002$ to 3.4 , with a median of $\langle z\rangle=0.56$. The rest-frame $10-40 \mathrm{keV}$ luminosities also span a wide range, from $L_{10-40 \mathrm{keV}} \approx 10^{39}$ to $10^{46} \mathrm{erg} \mathrm{s}^{-1}$, with a median value of $\left\langle L_{10-40 \mathrm{keV}}\right\rangle=10^{44.1} \mathrm{erg} \mathrm{s}^{-1}$. Previous X-ray missions with sensitivity at $>10 \mathrm{keV}$ were able to sample the AGN population below the knee of the $\mathrm{X}$-ray luminosity function $\left(L_{\star}\right)$ for redshifts up to $z \approx 0.05$, and NuSTAR extends this to $z \approx 1$; see Section 4.1.3.

7. We present the X-ray-optical flux plane for the optical $R$ band and the $8-24 \mathrm{keV}$ and $3-24 \mathrm{keV}$ NUSTAR bands. The majority of sources have $f_{\mathrm{X}} / f_{\mathrm{opt}}$ values consistent with those expected for AGNs based on the findings of previous low-energy $(<10 \mathrm{keV}) \mathrm{X}$-ray observatories. We find a strong, positive correlation between $f_{\mathrm{X}} / f_{\text {opt }}$ and $\mathrm{X}$-ray luminosity, in agreement with results at $<10 \mathrm{keV}$. We also find evidence for significant differences in $f_{\mathrm{X}} / f_{\text {opt }}$ between the BLAGNs and NLAGNs; see Section 4.2.1.

8. We measure a type 2 AGN fraction of $53_{-15}^{+14} \%$ for an effectively spectroscopically complete subset of the hardband $(8-24 \mathrm{keV})$ selected sample at $0.1<z<0.5$ and with $2 \times 10^{43}<L_{10-40 \mathrm{keV}}<2 \times 10^{44} \mathrm{erg} \mathrm{s}^{-1}$. Comparing with luminosity-matched $z<0.05$ AGNs selected by the Swift BAT survey, the NuSTAR-measured type 2 fraction for distant AGNs is higher, but consistent within the uncertainties. However, the NuSTAR-measured and Swift BAT-measured type 2 fractions appear to be systematically higher than those measured for redshiftand luminosity-matched AGNs selected by $<10 \mathrm{keV}$ $\mathrm{X}$-ray missions (e.g., Chandra and XMM-Newton); see Section 4.2.2. 
9. We compare the distribution of WISE $W 1-W 2$ and $W 2-W 3$ colors for NuSTAR AGNs with commonly applied MIR color-selection techniques. The fraction of NuSTAR AGNs that would be selected as AGNs based on the MIR colors alone is a strong function of X-ray luminosity, in agreement with findings for low-energy $(<10 \mathrm{keV}) \mathrm{X}$-ray-selected samples. The fraction of NuSTAR AGNs missed by MIR color selections is large, ranging between $\approx 15 \%$ and $\approx 80 \%$ for the highest luminosities $\left(L_{\mathrm{X}}>10^{44} \mathrm{erg} \mathrm{s}^{-1}\right)$ and the lowest luminosities $\left(L_{\mathrm{X}}<10^{43} \mathrm{erg} \mathrm{s}^{-1}\right)$, respectively; see Section 4.3.1. It is notable that a number of luminous NuSTARselected BLAGNs are not selected in the MIR, and that this appears to be driven by the intrinsic AGN properties; see Section 4.3.1.

10. We present the X-ray-MIR luminosity plane for sources that are AGN dominated at MIR wavelengths. For both the restframe $2-10 \mathrm{keV}$ and $10-40 \mathrm{keV}$ bands the large majority of the sources are consistent with being scattered around the intrinsic $L_{\mathrm{X}}-L_{6 \mu \mathrm{m}}$ relation; see Section 4.3.2. One source is highlighted as having an extremely low $L_{10-40 \mathrm{keV}} / L_{6 \mu \mathrm{m}}$ ratio $\left(\mathrm{J} 0650 ; \quad z=0.32 ; \quad L_{6 \mu \mathrm{m}} \approx 4 \times 10^{44} \mathrm{erg} \mathrm{s}^{-1}\right.$; $\left.L_{10-40 \mathrm{keV}}<2 \times 10^{43} \mathrm{erg} \mathrm{s}^{-1}\right)$. A detailed investigation reveals a narrow-line Seyfert 1, likely to be intrinsically X-ray weak as opposed to heavily obscured; see Section 4.3.2.

The NUSTAR serendipitous survey presented herein is the largest sample of distant AGNs selected with a focusing highenergy ( $\gtrsim 10 \mathrm{keV}$ ) X-ray observatory. As the NuSTAR science operations continue into the future, the serendipitous survey will continue to grow at a similar rate, and it is likely to eventually achieve a sample size on the order of $\gtrsim 1000$ sources. This will result in improved statistical constraints on the overall properties of the hard-X-ray-emitting source population and will facilitate the discovery of rare and extreme sources not sampled as effectively by the smaller-area dedicated NUSTAR surveys (e.g., in the COSMOS, ECDFS, EGS, GOODS-N, and UDS fields). A continued program of follow-up observations will be necessary to maximize the effectiveness of the serendipitous survey.

The authors first thank the anonymous referee for the constructive comments. We acknowledge financial support from the Science and Technology Facilities Council (STFC) grants ST/K501979/1 (G.B.L.), ST/I001573/1 (D.M.A.), and ST/J003697/2 (P.G.); a Herchel Smith Postdoctoral Fellowship of the University of Cambridge (G.B.L.); the ERC Advanced Grant FEEDBACK 340442 at the University of Cambridge (J.A.); a COFUND Junior Research Fellowship from the Institute of Advanced Study, Durham University (J.A.); the Leverhulme Trust (D.M.A.); CONICYT-Chile grants FONDECYT 1120061 and 1160999 (E.T.), 3140534 (S.S.), and Anillo ACT1101 (E.T. and F.E.B.); the Center of Excellence in Astrophysics and Associated Technologies (PFB 06; E.T. and F.E.B.); and the NASA Earth and Space Science Fellowship Program, grant NNX14AQ07H (M.B.). We extend gratitude to Felipe Ardila, Roberto Assef, Eduardo Bañados, Stanislav George Djorgovski, Andrew Drake, Jack Gabel, Audrey Galametz, Daniel Gawerc, David Girou, Marianne Heida, Nikita Kamraj, Peter Kosec, Thomas Krühler, Ashish Mahabal, Alessandro Rettura, and Aaron Stemo for their support during the ground-based follow-up observations. We thank John Lucey for unearthing the J1410 spectrum, and Sophie Reed, David Rosario, Mara Salvato, and Martin Ward for the informative discussions. Additional thanks to Eden Stern for lending a hand during the 2015 August Keck run. This work was supported under NASA Contract No. NNG08FD60C and made use of data from the NuSTAR mission, a project led by the California Institute of Technology, managed by the Jet Propulsion Laboratory, and funded by the National Aeronautics and Space Administration. We thank the NuSTAR Operations, Software and Calibration teams for support with the execution and analysis of these observations. This research has made use of the NUSTAR Data Analysis Software (NuSTARDAS) jointly developed by the ASI Science Data Center (ASDC, Italy) and the California Institute of Technology (USA).

Facilities: Chandra, ESO La Silla, Gemini, Keck, Magellan, NuSTAR, Palomar, SDSS, Swift, WISE, XMM-Newton.

\section{Appendix}

\section{A.1. Description of the NuSTAR Serendipitous Survey Source Catalog}

The NuSTAR serendipitous survey source catalog, containing 498 sources in total, is provided in electronic format. Here we describe the columns of the catalog, which are summarized in Table 5.

Column 1: the unique source identification numbers (ID), in order of increasing right ascension (R.A.).

Column 2: the unique NUSTAR source names, following the IAU-approved format: NuSTAR JHHMMSS \pm DDMM.m, where $\mathrm{m}$ is the truncated fraction of $1^{\prime}$ for the arcseconds component of the declination (decl.).

Columns 3, 4: the NuSTAR R.A. and decl. coordinates (J2000), as described in Section 2.3.

Columns 5-7: a binary flag indicating whether the source is detected with a false probability lower than our threshold of $\log \left(P_{\text {False }}\right)=-6$, for the soft $(3-8 \mathrm{keV})$, hard $(8-24 \mathrm{keV})$, and full $(3-24 \mathrm{keV})$ bands.

Columns 8-10: the same as columns 5-7, after deblending has been performed to account for contamination of the source counts from very nearby sources (see Section 2.4 of this paper, and Section 2.3.2 of M15). Deblending only affects a very small fraction of the overall sample (e.g., see Section 2.4).

Columns 11-13: the logarithm of the false probabilities $\left(P_{\text {False }}\right)$ of the NuSTAR-detected sources, for the three standard energy bands (see Section 2.3).

Columns 14-16: the same as columns 11-13, after deblending has been performed.

Column 17: a binary flag indicating whether the NuSTARdetected source remains significant after deblending, in at least one of the three standard energy bands.

Columns 18-32: photometric quantities, calculated at the source coordinates in columns 3 and 4, and using a source aperture of $30^{\prime \prime}$ radius (see Section 2.4). The values are nonaperture-corrected, i.e., they correspond to the $30^{\prime \prime}$ values, and have not been corrected to the full PSF values. We provide the total counts (i.e., all counts within the source aperture) and associated errors ( $84 \% \mathrm{CL}$ ), the background counts scaled to the source aperture, and the net source counts (i.e., total minus background) and associated errors. For the net source counts, we give $90 \%$ CL upper limits for sources not detected in a 
Table 5

Column Descriptions for the Primary NuSTAR Serendipitous Source Catalog

\begin{tabular}{|c|c|}
\hline Column Number & Description \\
\hline 1 & Unique source identification number (ID) \\
\hline 2 & Unique $N U S T A R$ source name \\
\hline 3,4 & Right ascension (R.A.) and declination (decl.) \\
\hline $5-7$ & Flags indicating the energy bands for which the source is detected \\
\hline $8-10$ & Same as columns $5-7$, post-deblending \\
\hline $11-13$ & Logarithm of the false probabilities for the three standard energy bands \\
\hline $14-16$ & Same as columns $11-13$, post-deblending \\
\hline 17 & Flag indicating whether the source is significant post-deblending, for at least one energy band \\
\hline $18-32$ & Total, background, and net source counts for the three standard energy bands, and associated errors \\
\hline $33-44$ & Same as columns $18-32$, post-deblending \\
\hline $45-47$ & Net vignetting-corrected exposure times at the source position, for the combined $\mathrm{A}+\mathrm{B}$ data \\
\hline $48-62$ & Total, background, and net source count rates for the three standard energy bands, and associated errors \\
\hline $63-68$ & Deblended net source count rates for the three standard energy bands, and associated errors \\
\hline $69-71$ & Band ratio and upper and lower errors \\
\hline $72-74$ & Effective photon index and upper and lower errors \\
\hline $75-80$ & Deblended fluxes in the three standard bands and associated errors \\
\hline 81 & Reference for the adopted lower-energy X-ray (Chandra, XMM-Newton, or Swift XRT) counterpart \\
\hline 82,83 & R.A. and decl. of the lower-energy X-ray counterpart \\
\hline 84 & Angular separation between the NUSTAR and lower-energy X-ray counterpart positions \\
\hline 85 & $3-8 \mathrm{keV}$ (3XMM or CSC) flux of the lower-energy X-ray counterpart \\
\hline 86 & Total 3-8 keV flux of all (3XMM or CSC) sources within $30^{\prime \prime}$ of the NUSTAR position \\
\hline 87,88 & R.A. and decl. of the adopted WISE counterpart \\
\hline 89 & Angular separation between the NUSTAR and WISE counterpart positions \\
\hline $90-97$ & WISE magnitudes in the four standard bands and associated errors \\
\hline 98 & Reference for the adopted optical counterpart \\
\hline 99, 100 & R.A. and decl. of the optical counterpart \\
\hline 101 & Angular separation between the NUSTAR and optical counterpart positions \\
\hline 102 & $R$-band magnitude for the optical counterpart \\
\hline 103 & Spectroscopic redshift \\
\hline 104 & Non-absorption-corrected, rest-frame $10-40 \mathrm{keV}$ luminosity \\
\hline 105 & Binary flag to indicate sources associated with the primary science targets of their respective NUSTAR fields \\
\hline 106 & Binary flag to indicate the sources used for the Aird et al. (2015a) study \\
\hline
\end{tabular}

(This table is available in its entirety in machine-readable form.)

given band. Throughout the table, upper limits are flagged with a -99 value in the error column.

Columns 33-44: the same as columns 18-32, after deblending has been performed.

Columns 45-47: the average net, vignetting-corrected exposure time at the source coordinates (columns 3 and 4), for each energy band. These correspond to the $\mathrm{A}+\mathrm{B}$ data, so they should be divided by 2 to obtain the average exposure per FPM. Units: s.

Columns 48-62: the non-aperture-corrected total, background, and net count rates (and associated errors; 84\% CL) determined from the photometric values in columns $18-32$, and the exposure times in columns 45-47. Units: $\mathrm{s}^{-1}$.

Columns 63-68: the deblended net count rates, and associated errors, determined from the photometric values in columns 33-44, and the exposure times in columns 45-47. Units: $\mathrm{s}^{-1}$.

Columns 69-71: the NuSTAR band ratio $\left(\mathrm{BR}_{\mathrm{Nu}}\right)$ and associated errors, as described in Section 2.4. Upper limits, lower limits, and sources with no constraints are flagged with $-99,-88$, and -77 values, respectively, in the error columns.

Columns 72-74: the effective photon index $\left(\Gamma_{\text {eff }}\right)$ and associated errors, estimated from the band ratio values in columns 69-71 (see Section 2.4).

Columns 75-80: the observed-frame fluxes and associated errors $(84 \% \mathrm{CL})$ for the three standard energy bands, after deblending has been performed. These are aperture-corrected values (i.e., they correspond to the full NuSTAR PSF) and are calculated from the count rates in columns 63-68 using the conversion factors listed in Section 2.4. Units: $\mathrm{erg} \mathrm{s}^{-1} \mathrm{~cm}^{-2}$.

Column 81: an abbreviated code indicating the origin of the adopted soft (i.e., low energy; $<10 \mathrm{keV}$ ) X-ray counterpart. CXO_CSC indicates counterparts from the CSC (Evans et al. 2010). XMM_3XMM indicates counterparts from the third XMM-Newton serendipitous source catalog (3XMM; Watson et al. 2009; Rosen et al. 2016). CXO_MAN, XMM_MAN, and XRT_MAN indicate sources manually identified using archival Chandra, XMM-Newton, and Swift XRT data, respectively. Section 3.1 details the counterpart matching.

Columns 82, 83: the R.A. and decl. coordinates (J2000) of the soft X-ray counterpart.

Column 84: the angular offset between the NUSTAR position (columns 3 and 4) and the soft X-ray counterpart position (columns 82 and 83). Units: arcseconds.

Column 85: the observed-frame $3-8 \mathrm{keV}$ flux of the soft $\mathrm{X}$-ray counterpart, for sources with counterparts in the CSC and 3XMM catalogs. For CSC sources we convert to the 3-8 keV flux from the $2-7 \mathrm{keV}$ flux using a conversion factor of 0.83 , and for the $3 \mathrm{XMM}$ sources we convert from the $4.5-12 \mathrm{keV}$ flux using a conversion factor of 0.92 . Units: erg s${ }^{-1} \mathrm{~cm}^{-2}$. 

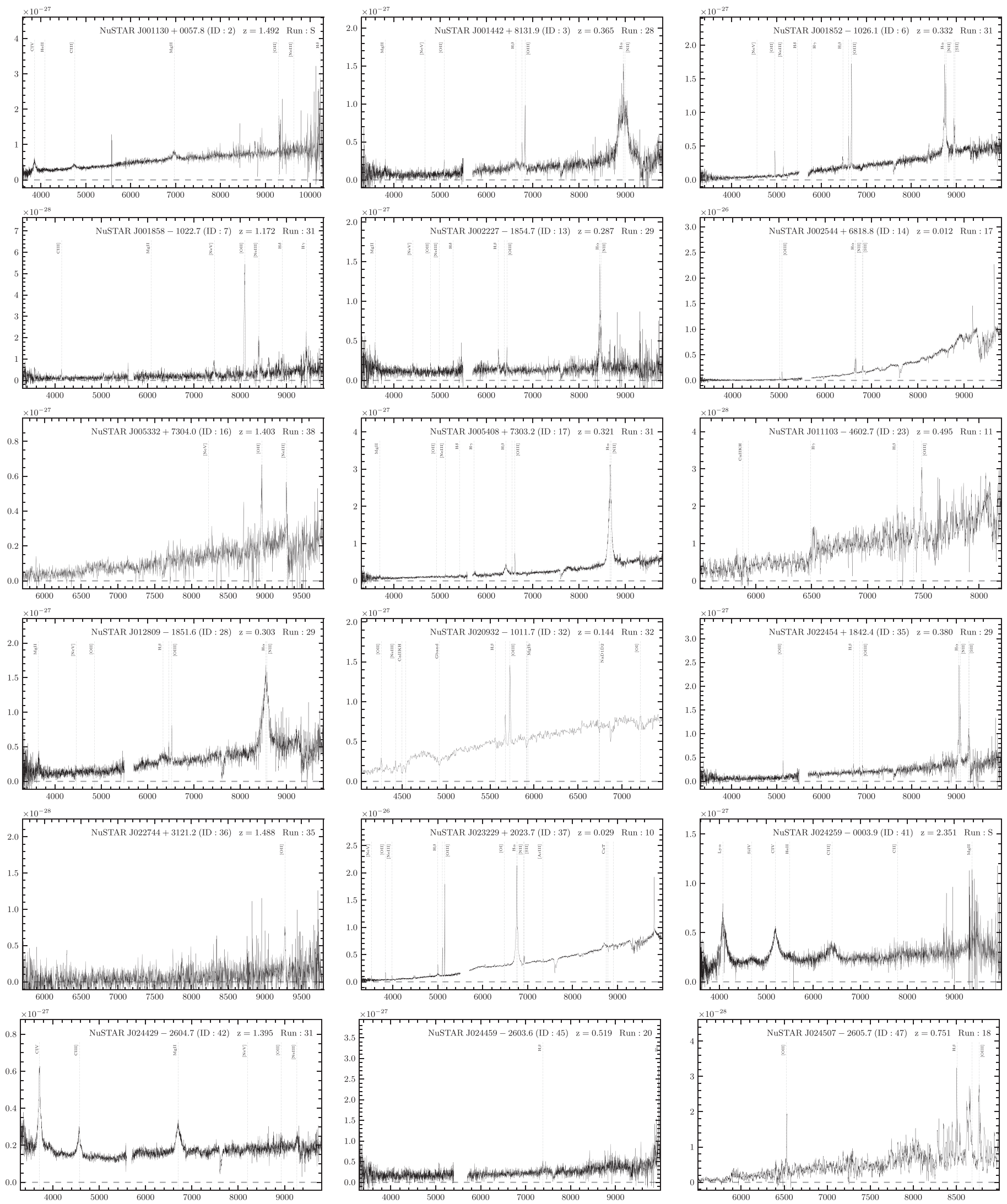

Figure 21. Optical spectra for the NUSTAR serendipitous survey sources. The horizontal axis shows the wavelength in units of $\AA$, and the vertical axis shows the flux $\left(f_{\nu}\right)$ in units of erg s${ }^{-1} \mathrm{~cm}^{-2} \mathrm{~Hz}^{-1}$. Shown in the upper right corner are the unique NuSTAR source name, the unique source ID, the source redshift, and the observing run identification number (corresponding to Tables 4 and 6; "S" indicates SDSS spectra). The identified emission and absorption lines are labeled and marked with vertical dashed gray lines.

(The complete figure set (261 images) is available.) 
Table 6

Summary of the Optical Spectroscopy for the NuSTAR Serendipitous Survey Sources.

\begin{tabular}{|c|c|c|c|c|c|c|}
\hline $\begin{array}{l}\text { ID } \\
(1)\end{array}$ & $\begin{array}{l}\text { NuSTAR Name } \\
\text { (2) }\end{array}$ & $\begin{array}{c}z \\
(3)\end{array}$ & $\begin{array}{l}\text { Type } \\
\text { (4) }\end{array}$ & $\begin{array}{l}\text { Lines } \\
(5)\end{array}$ & $\begin{array}{l}\text { Notes } \\
(6)\end{array}$ & $\begin{array}{l}\text { Run } \\
\text { (7) }\end{array}$ \\
\hline 1 & NuSTAR J000011-7652.5 & 0.053 & . . & & Jones et al. $(2004,2009)$ & $\mathrm{L}$ \\
\hline 2 & NuSTAR J001130+0057.8 & 1.492 & $\mathrm{BL}$ & $\mathrm{C}$ IV He II C III] Mg II [O II] [Ne III] $\mathrm{H} \delta$ & $\ldots$ & $\mathrm{S}$ \\
\hline 4 & NuSTAR J001542+8134.4 & $\cdots$ & . & $\cdots$ & Continuum detected & 33 \\
\hline 6 & NuSTAR J001852-1026.1 & 0.332 & BL & [Ne v] [O II] [Ne III] $\mathrm{H} \delta \mathrm{H} \gamma \mathrm{H} \beta$ [O III] $\mathrm{H} \alpha[\mathrm{N}$ II] [S II] & $\ldots$ & 31 \\
\hline
\end{tabular}

Note. The Columns are Described in Appendix A.2

(This table is available in its entirety in machine-readable form.)

Column 86: the total combined 3-8 keV flux of all (3XMM or CSC) sources within $30^{\prime \prime}$ of the NUSTAR position. Units: erg s $\mathrm{s}^{-1} \mathrm{~cm}^{-2}$.

Columns 87, 88: the R.A. and decl. coordinates (J2000) of the WISE counterpart, if there is a match in the WISE all-sky survey catalog (Wright et al. 2010). Section 3.2 details the WISE counterpart matching.

Column 89: the angular offset between the NUSTAR position (columns 3 and 4) and the WISE counterpart position (columns 87 and 88). Units: arcseconds.

Columns 90-97: the WISE profile-fit magnitudes (and associated errors), for the four standard WISE bands: $W 1$ $(\lambda \approx 3.4 \mu \mathrm{m}), \quad W 2 \quad(\approx 4.6 \mu \mathrm{m}), \quad W 3 \quad(\approx 12 \mu \mathrm{m})$, and $W 4$ $(\approx 22 \mu \mathrm{m})$. NaN error values indicate WISE upper limits. Units: Vega mag.

Column 98: an abbreviated code indicating the origin of the adopted optical counterpart to the NUSTAR source. The code SDSS indicates sources with soft X-ray counterparts and successful matches in the SDSS DR7 catalog (York et al. 2000). The code USNO indicates sources with soft X-ray counterparts and successful matches in the USNOB1 catalog (Monet et al. 2003). MAN indicates sources with a soft X-ray counterpart and a corresponding optical counterpart manually identified in the available optical coverage. SDSS_WISE and USNO_WISE indicate the cases where there is no soft X-ray counterpart to the NUSTAR position, but a WISE AGN candidate is identified within the $N u S T A R$ error circle and successfully matched to the SDSS DR7 or USNOB1 catalog (these are mainly used as candidates for spectroscopic followup). We give a detailed description of the procedure used to identify optical counterparts in Section 3.2.

Columns 99, 100: the R.A. and decl. coordinates (J2000) of the optical counterpart, for the sources with SDSS DR7 and USNOB1 matches.

Column 101: the angular offset between the NuSTAR position (columns 3 and 4) and the optical counterpart position (columns 99 and 100). Units: arcseconds.

Column 102: the $R$-band magnitude of the optical counterpart. For the SDSS DR7 matches, this is calculated as $R=r-0.16$. For the USNOB1 matches, this is taken as the mean of the two independent photographic plate measurements, R1mag and R2mag. For the manual identifications, the magnitude is taken from another optical catalog or manually determined from the imaging data. Units: Vega mag.

Column 103: the spectroscopic redshift of the NUSTAR source. The large majority of the redshifts were obtained through our own campaign of ground-based spectroscopic follow-up of NUSTAR serendipitous survey sources (see Section 3.3.1).

Column 104: the rest-frame $10-40 \mathrm{keV}$ luminosity, estimated from the fluxes in columns 75-80, following the procedure outlined in Section 2.4. Negative values indicate upper limits. The luminosities are observed values, uncorrected for any absorption along the line of sight. The intrinsic luminosities may therefore be higher, for highly absorbed AGNs. Units: erg $\mathrm{s}^{-1}$.

Column 105: a binary flag indicating the few sources that show evidence for being associated with the primary science targets of their respective $N U S T A R$ observations, according to the definition in Section $2.3[\Delta(c z)<0.05 c z]$.

Column 106: a binary flag highlighting the sources used in the Aird et al. (2015a) study.

\section{A.2. Optical Spectroscopic Properties of Individual Objects}

Here we provide details of the optical spectroscopic properties of individual sources from the NuSTAR serendipitous survey. As described in Section 3.3, these largely result from our dedicated follow-up campaign using the Keck, Magellan, NTT, and Palomar facilities, and also from existing publicly available spectroscopy (primarily SDSS spectroscopy). Individual source spectra $\left(F_{\nu}\right.$ versus $\left.\lambda\right)$ are shown in Figure Set 21, and details for individual sources are tabulated in Table 6, the columns of which are as follows: columns (1) and (2) give the unique source identification number and the unique NuSTAR source name, as listed in the source catalog; columns (3) and (4) give the source redshift and classification (see Section 3.3.2); column (5) lists the emission or absorption lines identified (the latter are marked with $\dagger$ symbols), which are additionally highlighted in the individual components of Figure Set 21; column (6) gives individual object notes, including references for literature spectra; and column (7) gives the unique observing run identification number, as defined in Table 4 ("S" and "L" mark spectra obtained from the SDSS and from elsewhere in the literature, respectively).

\section{A.3. Assessment of Spurious Optical and IR Counterpart Matches}

Figure 22 shows histograms of the radial offsets between soft X-ray counterpart (Chandra, Swift XRT, and XMM-Newton) positions and the optical (SDSS and USNOB1) and IR (WISE) matches. We compare to the radial offset distributions expected for spurious matches, given the sky density of sources in the IR and optical surveys, in order to estimate spurious matching fractions. 

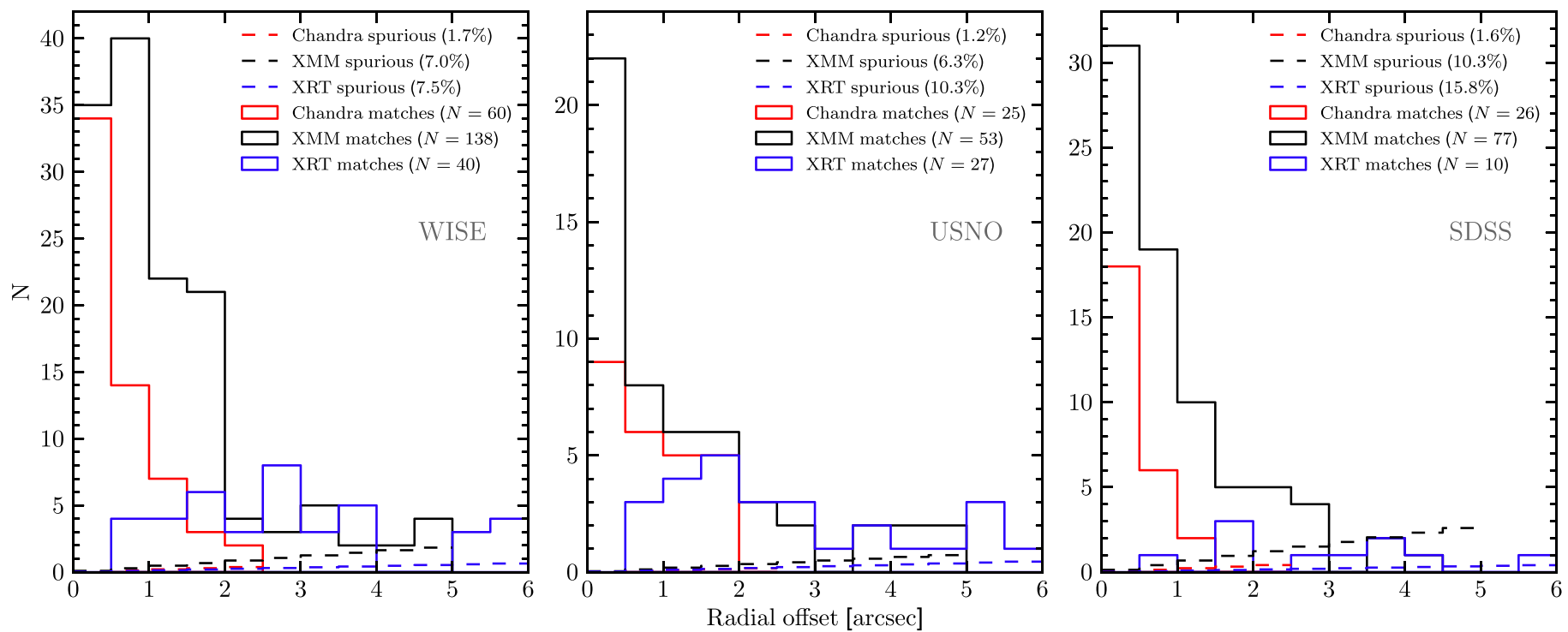

Figure 22. Histograms showing the distributions of positional offsets between the soft X-ray (Chandra, XMM-Newton, and Swift XRT; red, black, and blue solid lines, respectively) positions and the matched WISE (left panel), USNOB1 (middle panel), and SDSS (right panel) counterparts, for the NuSTAR serendipitous survey sources with $|b|>10^{\circ}$. The dashed lines show the distributions expected for spurious matches (i.e., assuming that there are no true IR or optical counterparts to the $\mathrm{X}$-ray sources); these are calculated using the IR and optical source densities, taking median values across the range of sky positions for the NuSTAR serendipitous survey sources: $3.2 \mathrm{arcmin}^{-2}$ for WISE, $3.3 \mathrm{arcmin}^{-2}$ for USNOB1, and $8.1 \operatorname{arcmin}^{-2}$ for SDSS. The bracketed percentages show the inferred spurious fraction (i.e., the fraction of the X-ray sources with false IR or optical matches) for each subsample.

\section{A.4. Assessment of Spectroscopic Completeness for the Type 2 Fraction Subsample}

Here we assess the effective spectroscopic completeness of the subset of the NUSTAR serendipitous survey sample used to measure the type 2 fraction (see Section 4.2.2). The subset is limited to hard-band (8-24 keV) selected sources at redshifts of $0.1<z<0.5$ and luminosities of $2 \times 10^{43}<L_{10-40 \mathrm{keV}}<$ $2 \times 10^{44} \mathrm{erg} \mathrm{s}^{-1}$ and includes 30 spectroscopically identified sources (all NLAGNs or BLAGNs). To assess the completeness, we must also consider the unidentified sources that may or may not lie within these redshift and luminosity ranges (i.e., sources labeled as crosses in Figure 14), and their reasons for lacking successful spectroscopic follow-up. Since the majority of the spectroscopically identified sources in this subsample lie at $R<20$, we consider all unidentified sources with $R<20$ (we conservatively include sources with lower limits in $R$ ) as potentially lying within the redshift and luminosity ranges stated above. There are 10 such unidentified sources in total. This includes one likely BL Lac type object that we exclude owing to the possibility of beaming. A further four of the unidentified sources can be safely excluded without biasing the type 2 fraction measurement: one of these has an unambiguous optical counterpart, and simply has not yet been targeted with ground-based facilities; two have not been targeted owing to the lack of Chandra or XMM-Newton coverage, required to distinguish between multiple optical counterparts within the large $N U S T A R$ positional error circle; and for one obtaining spectroscopy is problematic owing to the close proximity of brighter optical sources. There are five remaining unidentified sources to consider, where follow-up has not been performed owing to the lack of a detection in the available Chandra and/ or XMM-Newton coverage (and therefore the lack of an accurate X-ray position). Four of these have high $P_{\text {False }}$ values at $8-24 \mathrm{keV}\left(\log P_{\text {False }}=-6.5\right.$ to -6.0 , i.e., close to our detection threshold of $\log P_{\text {False }}=-6.0$ ) and comparably deep Chandra or XMM-Newton coverage, which indicates that they are likely to be spurious sources. The remaining single source is strongly detected at $8-24 \mathrm{keV}\left(\log P_{\text {False }}=-9.6\right)$ and has relatively low quality Chandra and XMM-Newton coverage, thus consistent with being a genuine astrophysical source. We therefore consider the effective spectroscopic completeness of this subsample to be $97 \%-100 \%$ (i.e., $30 / 31$ or $30 / 30$, depending on whether the final source lies above or below $R=20$, since there is only a lower limit in $R$ ).

\section{A.5. J0650-a Low- $L_{\mathrm{X}} / L_{\mathrm{MIR}}$, Likely X-Ray-weak NLSyl}

Here we consider an outlier in X-ray-to-MIR luminosity ratio, NuSTAR J065003+6046.8 (hereafter J0650; $z=0.319$ ). For this source, the upper limit of $L_{10-40 \mathrm{keV}} / L_{6 \mu \mathrm{m}}<0.05$ lies below the CT AGN threshold (as shown in Figure 20). In other words, the hard X-ray luminosity is very weak compared to that expected based on the MIR luminosity $\left(L_{6 \mu \mathrm{m}}=3.7 \times 10^{44} \mathrm{erg} \mathrm{s}^{-1}\right)$. The source is not detected in the full and hard NUSTAR bands, but is weakly detected in the soft band $\left(\log P_{\text {False }}=-6.9 ; \approx 25\right.$ net source counts, for an effective exposure time of $16 \mathrm{ks}$ ), suggesting a relatively steep spectral slope. The properties of the counterparts at X-ray, IR, and optical wavelengths (see below) add confidence that the NUSTAR detection is not spurious. J0650 has a strongly detected XMM-Newton counterpart, the $0.5-10 \mathrm{keV}$ spectrum of which has 107 net source counts (for an $8 \mathrm{ks}$ exposure). A powerlaw fit provides a statistically acceptable fit to the XMM-Newton spectrum $(C / n=139 / 159)$, and the photon index is constrained to be $\Gamma_{\text {eff }}=3.1 \pm 0.6$, which is very steep and above the typical range observed for AGNs. For the $3-10 \mathrm{keV}$ band, where $N U S T A R$ and XMM-Newton overlap in sensitivity, the source is undetected with XMM-Newton, with $<19.5$ EPIC counts overall and a 3-8 keV flux upper limit of $<1.5 \times 10^{-14} \mathrm{erg} \mathrm{s}^{-1} \mathrm{~cm}^{-2}$ (99\% CL). This is significantly lower than our photometric NUSTAR flux of $4.8 \pm 1.6 \times 10^{-14} \mathrm{erg} \mathrm{s}^{-1} \mathrm{~cm}^{-2}$ in the $3-8 \mathrm{keV}$ band. The disagreement could in part result from X-ray variability (between the 2003 XMM-Newton observation and the 2014 NuSTAR observation), which is especially likely in this case given 
Table 7

Column Descriptions for the Secondary NuSTAR Serendipitous Source Catalog

\begin{tabular}{ll}
\hline \hline Column Number & Description \\
\hline 1 & Unique source identification number (ID) \\
2 & Unique NuSTAR source name \\
3,4 & Right ascension (R.A.) and declination (decl.) \\
$5-16$ & Total, background, and net source counts for the three standard energy bands, and associated errors \\
$17-19$ & Net vignetting-corrected exposure times at the source position, for the combined A+B data \\
$20-25$ & Net source count rates for the three standard energy bands, and associated errors \\
$26-31$ & Fluxes in the three standard bands and associated errors \\
$32-34$ & Observatory name and coordinates (R.A. and decl.) for the lower-energy X-ray counterpart \\
$35-37$ & Reference and coordinates (R.A. and decl.) for the optical or WISE counterpart \\
38 & Spectroscopic redshift \\
39 & Non-absorption-corrected, rest-frame 10-40 keV luminosity \\
40 & Character indicating the reason for not appearing in the primary catalog \\
\hline
\end{tabular}

(This table is available in its entirety in machine-readable form.)

the NLSy1 optical classification (see below). The NUSTAR flux is also likely boosted by the Eddington bias, which we have established to be significant at this low $3-8 \mathrm{keV}$ flux level (see Figure 5, and C15).

The X-ray luminosities measured at high and low energies are $L_{10-40 \mathrm{keV}}^{\mathrm{obs}}<2.0 \times 10^{43} \mathrm{erg} \mathrm{s}^{-1}$ (from NuSTAR photometry) and $L_{2-10 \mathrm{keV}}^{\mathrm{obs}}=2.6 \times 10^{42} \mathrm{erg} \mathrm{s}^{-1}$ (from the XMM-Newton spectrum), respectively. Given the AGN $6 \mu \mathrm{m}$ luminosity measured from WISE photometry $\left(L_{6 \mu \mathrm{m}}=3.7 \times 10^{44} \mathrm{erg} \mathrm{s}^{-1}\right.$; the source is AGN dominated at this wavelength based on the WISE colors), these suggest comparatively low X-ray-to-MIR luminosity ratios, with the $2-10 \mathrm{keV}$ and $10-40 \mathrm{keV}$ X-ray luminosities potentially suppressed by factors of $\approx 50$ and $\gtrsim 7$, respectively, with respect to the intrinsic relations for AGNs (see Figure 20). In the case of the $2-10 \mathrm{keV}$ luminosity, the low value is in part due to the relatively steep soft X-ray spectral slope. If the apparent X-ray suppression were due to AGN absorption, we would expect a flat X-ray spectral slope $\left(\Gamma_{\text {eff }}<1\right)$, but the observed spectral slope is comparatively steep $\left(\Gamma_{\text {eff }} \approx 3\right)$. One possibility is that the source is an intrinsically X-ray-weak, unobscured AGN. As described below, the source shows the properties of an NLSy1 in the optical, and intrinsic X-ray weakness has been identified for some objects in this class (e.g., Leighly et al. 2007a, 2007b; Miniutti et al. 2012).

Further evidence for the presence of an AGN in J0650 is given by the WISE colors, which place it firmly within the MIR AGN selection regions $(W 1-W 2=1.2 ; W 2-W 3=3.2)$. The source is also comparatively bright in the longer-wavelength $W I S E$ bands $(W 3=9.50 \pm 0.03$ and $W 4=7.24 \pm 0.07)$. On the basis of our results for WISE colors as a function of X-ray luminosity (Section 4.3.1), J0650 is statistically highly likely to have an intrinsic X-ray luminosity of $L_{10-40 \mathrm{keV}}^{\text {int }}>10^{44} \mathrm{erg} \mathrm{s}^{-1}$. The fact that the observed X-ray luminosity is so much lower may be explained by a combination of intrinsic X-ray weakness and the steep spectral slope at low energies, the latter of which may result in a relative increase in the dust-heating photons that are reprocessed into the MIR waveband.

Key information for this object is provided by our Keck spectrum, which reveals a likely NLSy1 AGN. We detect multiple strong emission lines, from $\mathrm{Mg}$ II at the blue end to $\mathrm{H} \alpha$ and $[\mathrm{N}$ II] at the red end. The source satisfies the conventional NLSy1 definition, with a relatively narrow $\mathrm{H} \beta$ line $\left(\mathrm{FWHM} \approx 1710 \mathrm{~km} \mathrm{~s}^{-1}\right)$ and a low $[\mathrm{O} \mathrm{III]} \lambda 5007 / \mathrm{H} \beta$ flux ratio (e.g., Goodrich 1989). There are also multiple strong Fe II emission lines, another characteristic feature of NLSy1s (e.g., Zhou et al. 2006). Notably, the [O III] $\lambda 5007$ line is contaminated by strong Fe emission. NLSy1s are associated with low black hole masses and high accretion rates (e.g., Pounds et al. 1995; Boller et al. 1996; Mathur 2000) and typically have significantly steeper X-ray spectral slopes than normal unobscured AGNs (e.g., Boller et al. 1996; Brandt et al. 1997). The latter property is congruous with our measurement of an extremely steep X-ray photon index for J0650 ( $\left.\Gamma_{\text {eff }} \approx 3\right)$.

\section{A.6. Description of the Secondary Source Catalog}

Here we provide a secondary catalog of $64 \mathrm{NuSTAR}$ sources identified using an independent source detection approach. This independent (or "secondary") method uses wavdetect to search for significant emission peaks in the FPMA and FPMB data separately (see Section 2.1.1 of Alexander et al. 2013) and in the combined $\mathrm{A}+\mathrm{B}$ data. The method was developed alongside the primary one (Section 2.3 of this paper) in order to investigate the optimum source detection methodologies for $N U S T A R$ and to identify sources in regions of the NuSTAR coverage that are automatically excluded in the primary source detection. We emphasize that these secondary sources are not used in any of the science analyses presented in this paper. The results in this work therefore correspond to a single, welldefined sample. Nevertheless, these secondary sources are robust $N U S T A R$ detections, some of which will be incorporated in future NuSTAR studies (e.g., Chen et al. 2017; J. A. Tomsick et al. 2017, in preparation), and many for which (35 out of the 43 sources with spectroscopic identifications) we have obtained new spectroscopic redshifts and classifications through our follow-up program.

The columns for the secondary source catalog are summarized in Table 7. The NUSTAR columns are equivalent to the primary catalog columns described in Section A.1, with the exception that the count rates (columns 20-25) are aperturecorrected values. The photometric columns are blank where the $\mathrm{A}+\mathrm{B}$ data prohibit reliable photometric constraints. The final column assigns a character to each source, indicating the reason for not being included in the primary catalog. These are categorized into four groups: (E) the source is within or very close to the peripheral region of the NUSTAR mosaic, which is excluded from the primary source detection (33\% of cases); (T) 
Table 8

Summary of the Optical Spectroscopy for the Secondary Catalog Sources

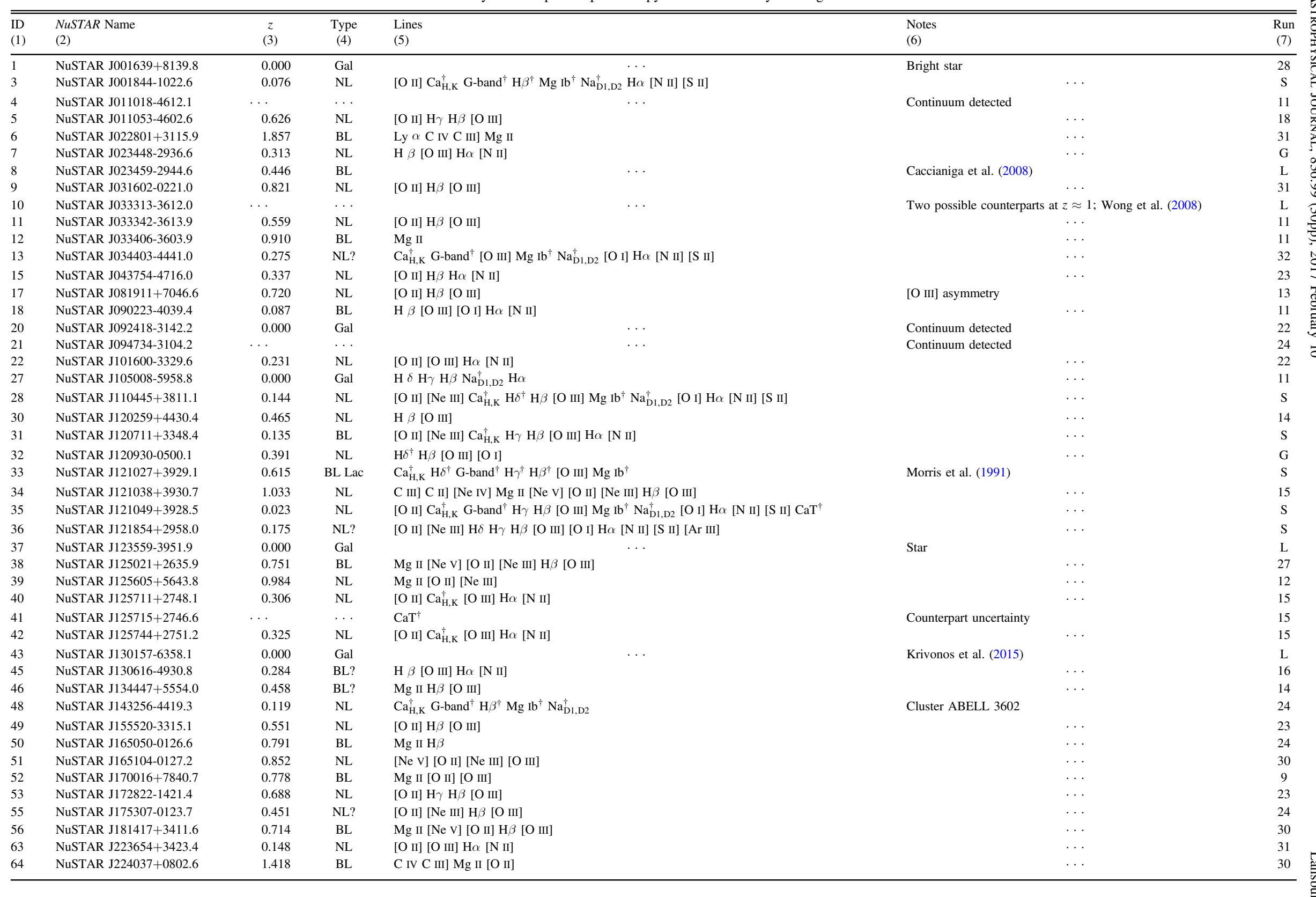

(This table is available in machine-readable form.) 

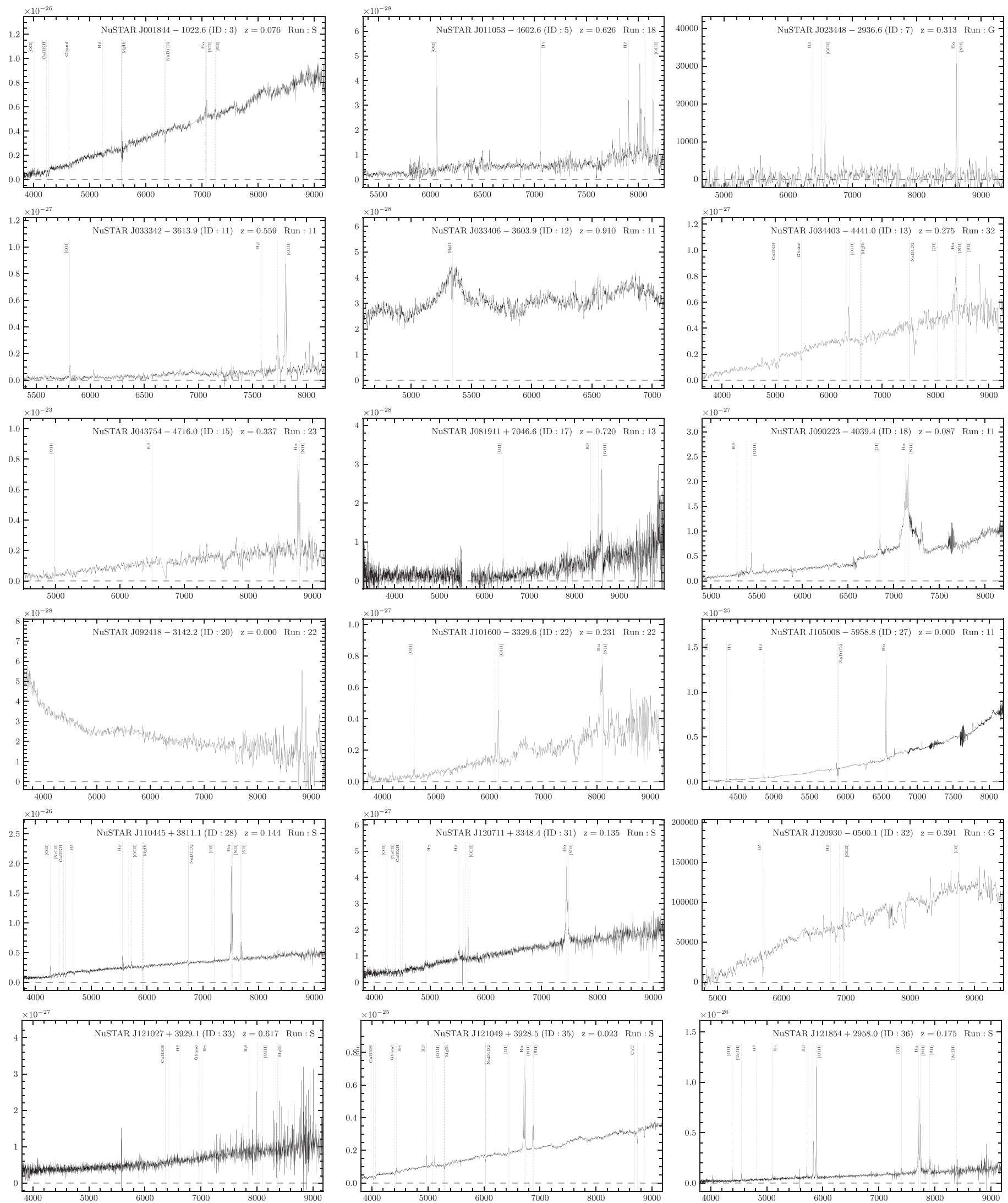

Figure 23. Optical spectra for the secondary catalog sources. The axes and labeling are the same as for Figure Set 21.

(The complete figure set (30 images) is available.) 
the source is narrowly offset from the central science target position for the NUSTAR observation (and thus automatically excluded; see Section 2.3), or from another bright source in the field $(11 \%)$; $(\mathrm{X})$ the source lies in a region that is masked out, or in a NUSTAR field that is excluded from the primary source detection (44\%; e.g., due to highly contaminating stray light or a bright science target); or (L) the source has a comparatively low detection significance (12\%).

In Table 8 we tabulate details of the optical spectroscopic properties of individual sources from the secondary catalog with spectroscopic coverage. The columns are equivalent to those in Table 6, with the exception of an additional observing run label ("G") to mark sources followed up with Gemini-South in January and February of 2016. For 78\% of the sources in Table 8 the spectroscopic constraints are from our dedicated follow-up program (with Keck, Palomar, NTT, Magellan, and Gemini), and for $22 \%$ they are from the SDSS or the literature. Individual source spectra $\left(F_{\nu}\right.$ versus $\left.\lambda\right)$ are shown in Figure Set 23.

\section{References}

Aird, J., Alexander, D. M., Ballantyne, D. R., et al. 2015a, ApJ, 815, 66 Aird, J., Coil, A. L., Georgakakis, A., et al. 2015b, MNRAS, 451, 1892 Ajello, M., Greiner, J., Kanbach, G., et al. 2008, ApJ, 678, 102 Akiyama, M., Ohta, K., Yamada, T., et al. 2000, ApJ, 532, 700 Alexander, D. M., Bauer, F. E., Brandt, W. N., et al. 2003, AJ, 126, 539 Alexander, D. M., Brandt, W. N., Hornschemeier, A. E., et al. 2001, AJ, 122,2156

Alexander, D. M., \& Hickox, R. C. 2012, NewAR, 56, 93

Alexander, D. M., Stern, D., Del Moro, A., et al. 2013, ApJ, 773, 125

Asmus, D., Gandhi, P., Hönig, S. F., Smette, A., \& Duschl, W. J. 2015, MNRAS, 454, 766

Assef, R. J., Kochanek, C. S., Brodwin, M., et al. 2010, ApJ, 713, 970

Assef, R. J., Stern, D., Kochanek, C. S., et al. 2013, ApJ, 772, 26

Ballantyne, D. R., Everett, J. E., \& Murray, N. 2006, ApJ, 639, 740

Baloković, M., Comastri, A., Harrison, F. A., et al. 2014, ApJ, 794, 111

Barcons, X., Carrera, F. J., Ceballos, M. T., et al. 2007, A\&A, 476, 1191

Barger, A. J., Cowie, L. L., Capak, P., et al. 2003, AJ, 126, 632

Baumgartner, W. H., Tueller, J., Markwardt, C. B., et al. 2013, ApJS, 207, 19 Bertin, E., \& Arnouts, S. 1996, A\&AS, 117, 393

Boller, T., Brandt, W. N., \& Fink, H. 1996, A\&A, 305, 53

Bottacini, E., Ajello, M., \& Greiner, J. 2012, ApJS, 201, 34

Brandt, W. N., \& Alexander, D. M. 2015, A\&ARv, 23, 1

Brandt, W. N., \& Hasinger, G. 2005, ARA\&A, 43, 827

Brandt, W. N., Mathur, S., \& Elvis, M. 1997, MNRAS, 285, L25

Burlon, D., Ajello, M., Greiner, J., et al. 2011, ApJ, 728, 58

Caccianiga, A., Severgnini, P., Della Ceca, R., et al. 2008, A\&A, 477, 735

Cardamone, C. N., Urry, C. M., Damen, M., et al. 2008, ApJ, 680, 130

Chen, C.-T. J., Brandt, W. N., Reines, A. E., et al. 2017, arXiv:1701.08768

Civano, F., Hickox, R. C., Puccetti, S., et al. 2015, ApJ, 808, 185

Comastri, A., Fiore, F., Vignali, C., et al. 2001, MNRAS, 327, 781

Comastri, A., Vignali, C., Brusa, M. \& Hellas2XMM Consortia 2002, in ASP Conf. Ser. 284, IAU Coll. 184: AGN Surveys, ed. R. F. Green, E. Y. Khachikian, \& D. B. Sanders (San Francisco, CA: ASP), 235

Della Ceca, R., Castelli, G., Braito, V., Cagnoni, I., \& Maccacaro, T. 1999, ApJ, 524, 674

Del Moro, A., Mateos, S., Watson, M. G., \& Akiyama, M. 2008, in 8th National Conf. on AGN, ed. L. Lanteri et al.

Del Moro, A., Mullaney, J. R., Alexander, D. M., et al. 2014, ApJ, 786, 16

Dickinson, M., Giavalisco, M. \& GOODS Team 2003, in The Mass of Galaxies at Low and High Redshift, ed. R. Bender \& A. Renzini (Berlin: Springer), 324

Eckart, M. E., McGreer, I. D., Stern, D., Harrison, F. A., \& Helfand, D. J. 2010, ApJ, 708, 584

Eckart, M. E., Stern, D., Helfand, D. J., et al. 2006, ApJS, 165, 19

Evans, I. N., Primini, F. A., Glotfelty, K. J., et al. 2010, ApJS, 189, 37

Evans, P. A., Osborne, J. P., Beardmore, A. P., et al. 2014, ApJS, 210, 8

Fabian, A. C., \& Barcons, X. 1992, ARA\&A, 30, 429

Fiore, F., Brusa, M., Cocchia, F., et al. 2003, A\&A, 409, 79

Fiore, F., Giommi, P., Vignali, C., et al. 2001, MNRAS, 327, 771

Fiore, F., Puccetti, S., Brusa, M., et al. 2009, ApJ, 693, 447
Freeman, P. E., Kashyap, V., Rosner, R., \& Lamb, D. Q. 2002, ApJS, 138, 185 Fruscione, A., McDowell, J. C., Allen, G. E., et al. 2006, Proc. SPIE, 6270 , $62701 \mathrm{~V}$

Gandhi, P., Crawford, C. S., Fabian, A. C., \& Johnstone, R. M. 2004, MNRAS, 348,529

Gandhi, P., Horst, H., Smette, A., et al. 2009, A\&A, 502, 457

Gandhi, P., Lansbury, G. B., Alexander, D. M., et al. 2014, ApJ, 792, 117

Gehrels, N. 1986, ApJ, 303, 336

Giacconi, R., Bechtold, J., Branduardi, G., et al. 1979, ApJL, 234, L1

Gioia, I. M., Maccacaro, T., Schild, R. E., et al. 1990, ApJS, 72, 567

Goodrich, R. W. 1989, ApJ, 342, 224

Groth, E. J., Kristian, J. A., Lynds, R., et al. 1994, BAAS, 26, 1403

Hao, H., Elvis, M., Civano, F., et al. 2010, ApJL, 724, L59

Harrison, F. A., Aird, J., Civano, F., et al. 2016, ApJ, 831, 185

Harrison, F. A., Craig, W. W., Christensen, F. E., et al. 2013, ApJ, 770, 103

Harrison, F. A., Eckart, M. E., Mao, P. H., Helfand, D. J., \& Stern, D. 2003 , ApJ, 596, 944

Hasinger, G. 2008, A\&A, 490, 905

Hasinger, G., Miyaji, T., \& Schmidt, M. 2005, A\&A, 441, 417

Hickox, R. C., \& Markevitch, M. 2006, ApJ, 645, 95

Hong, J., Mori, K., Hailey, C. J., et al. 2016, ApJ, 825, 132

Hornschemeier, A. E., Brandt, W. N., Garmire, G. P., et al. 2001, ApJ, 554,742

Ichikawa, K., Ueda, Y., Terashima, Y., et al. 2012, ApJ, 754, 45

Jarrett, T. H., Cohen, M., Masci, F., et al. 2011, ApJ, 735, 112

Jones, D. H., Read, M. A., Saunders, W., et al. 2009, MNRAS, 399, 683

Jones, D. H., Saunders, W., Colless, M., et al. 2004, MNRAS, 355, 747

Jun, H. D., \& Im, M. 2013, ApJ, 779, 104

Kim, D.-W., Cameron, R. A., Drake, J. J., et al. 2004, ApJS, 150, 19

Komatsu, E., Smith, K. M., Dunkley, J., et al. 2011, ApJS, 192, 18

Koss, M., Mushotzky, R., Baumgartner, W., et al. 2013, ApJL, 765, L26

Kraft, R. P., Burrows, D. N., \& Nousek, J. A. 1991, ApJ, 374, 344

Krivonos, R., Revnivtsev, M., Lutovinov, A., et al. 2007, A\&A, 475, 775

Krivonos, R. A., Tsygankov, S. S., Lutovinov, A. A., et al. 2015, ApJ, 809, 140

Laird, E. S., Nandra, K., Georgakakis, A., et al. 2009, ApJS, 180, 102

La Franca, F., Fiore, F., Comastri, A., et al. 2005, ApJ, 635, 864

LaMassa, S. M., Ricarte, A., Glikman, E., et al. 2016, ApJ, 820, 70

Lansbury, G. B., Alexander, D. M., Del Moro, A., et al. 2014, ApJ, 785, 17

Lansbury, G. B., Gandhi, P., Alexander, D. M., et al. 2015, ApJ, 809, 115

Lanzuisi, G., Piconcelli, E., Fiore, F., et al. 2009, A\&A, 498, 67

Lanzuisi, G., Ranalli, P., Georgantopoulos, I., et al. 2015, A\&A, 573, A137

Lawrence, A., Warren, S. J., Almaini, O., et al. 2007, MNRAS, 379, 1599

Lehmann, I., Hasinger, G., Schmidt, M., et al. 2001, A\&A, 371, 833

Lehmer, B. D., Brandt, W. N., Alexander, D. M., et al. 2005, ApJS, 161, 21

Leighly, K. M., Halpern, J. P., Jenkins, E. B., et al. 2007a, ApJ, 663, 103

Leighly, K. M., Halpern, J. P., Jenkins, E. B., \& Casebeer, D. 2007b, ApJS, 173,1

Luo, B., Brandt, W. N., Alexander, D. M., et al. 2014, ApJ, 794, 70

Lutz, D., Maiolino, R., Spoon, H. W. W., \& Moorwood, A. F. M. 2004, A\&A, 418, 465

Maccacaro, T., Gioia, I. M., Wolter, A., Zamorani, G., \& Stocke, J. T. 1988, ApJ, 326, 680

Malizia, A., Bassani, L., Bazzano, A., et al. 2012, MNRAS, 426, 1750

Markowitz, A. G., Krumpe, M., \& Nikutta, R. 2014, MNRAS, 439, 1403

Massaro, E., Giommi, P., Leto, C., et al. 2009, A\&A, 495, 691

Mateos, S., Alonso-Herrero, A., Carrera, F. J., et al. 2012, MNRAS, 426, 3271

Mateos, S., Alonso-Herrero, A., Carrera, F. J., et al. 2013, MNRAS, 434, 941

Mateos, S., Carrera, F. J., Alonso-Herrero, A., et al. 2015, MNRAS, 449,1422

Mateos, S., Warwick, R. S., Carrera, F. J., et al. 2008, A\&A, 492, 51

Mathur, S. 2000, MNRAS, 314, L17

Matsuta, K., Gandhi, P., Dotani, T., et al. 2012, ApJ, 753, 104

McHardy, I. M., Gunn, K. F., Newsam, A. M., et al. 2003, MNRAS, 342, 802

Merloni, A., Bongiorno, A., Brusa, M., et al. 2014, MNRAS, 437, 3550

Miniutti, G., Brandt, W. N., Schneider, D. P., et al. 2012, MNRAS, 425, 1718

Monet, D. G., Levine, S. E., Canzian, B., et al. 2003, AJ, 125, 984

Moretti, A., Perri, M., Capalbi, M., et al. 2006, A\&A, 448, L9

Mori, K., Hailey, C. J., Krivonos, R., et al. 2015, ApJ, 814, 94

Morris, S. L., Stocke, J. T., Gioia, I. M., et al. 1991, ApJ, 380, 49

Mullaney, J. R., Del-Moro, A., Aird, J., et al. 2015, ApJ, 808, 184

Mushotzky, R. F., Cowie, L. L., Barger, A. J., \& Arnaud, K. A. 2000, Natur, 404, 459

Nandra, K., Georgantopoulos, I., Ptak, A., \& Turner, T. J. 2003, ApJ, 582, 615

Nandra, K., Laird, E. S., Adelberger, K., et al. 2005, MNRAS, 356, 568

Pounds, K. A., Done, C., \& Osborne, J. P. 1995, MNRAS, 277, L5

Ricci, C., Ueda, Y., Koss, M. J., et al. 2015, ApJL, 815, L13 
Risaliti, G., Elvis, M., Fabbiano, G., Baldi, A., \& Zezas, A. 2005, ApJL, 623, L93

Rosen, S. R., Webb, N. A., Watson, M. G., et al. 2016, A\&A, 590, A1

Schmidt, M., \& Green, R. F. 1983, ApJ, 269, 352

Schmidt, M., Hasinger, G., Gunn, J., et al. 1998, A\&A, 329, 495

Scoville, N., Aussel, H., Brusa, M., et al. 2007, ApJS, 172, 1

Stern, D. 2015, ApJ, 807, 129

Stern, D., Assef, R. J., Benford, D. J., et al. 2012, ApJ, 753, 30

Stern, D., Lansbury, G. B., Assef, R. J., et al. 2014, ApJ, 794, 102

Stickel, M., Kuehr, H., \& Fried, J. W. 1993, A\&AS, 97, 483

Tingay, S. J., Jauncey, D. L., King, E. A., et al. 2003, PASJ, 55, 351

Tozzi, P., Rosati, P., Nonino, M., et al. 2001, ApJ, 562, 42

Treister, E., \& Urry, C. M. 2006, ApJL, 652, L79

Treister, E., Urry, C. M., Chatzichristou, E., et al. 2004, ApJ, 616, 123

Tueller, J., Mushotzky, R. F., Barthelmy, S., et al. 2008, ApJ, 681, 113
Ueda, Y., Ishisaki, Y., Takahashi, T., Makishima, K., \& Ohashi, T. 2005, ApJS, 161, 185

Ueda, Y., Takahashi, T., Inoue, H., et al. 1999, ApJ, 518, 656

Vasudevan, R. V., Brandt, W. N., Mushotzky, R. F., et al. 2013, ApJ, 763, 111

Watson, M. G., Schröder, A. C., Fyfe, D., et al. 2009, A\&A, 493, 339

Wik, D. R., Hornstrup, A., Molendi, S., et al. 2014, ApJ, 792, 48

Wong, D. S., Chornock, R., \& Filippenko, A. V. 2008, PASP, 120, 266

Worsley, M. A., Fabian, A. C., Bauer, F. E., et al. 2005, MNRAS, 357, 1281

Wright, E. L., Eisenhardt, P. R. M., Mainzer, A. K., et al. 2010, AJ, 140,1868

Xue, Y. Q., Luo, B., Brandt, W. N., et al. 2011, ApJS, 195, 10

Xue, Y. Q., Wang, S. X., Brandt, W. N., et al. 2012, ApJ, 758, 129

York, D. G., Adelman, J., Anderson, J. E., Jr., et al. 2000, AJ, 120, 1579

Younes, G., Kouveliotou, C., Kargaltsev, O., et al. 2012, ApJ, 757, 39

Zhou, H., Wang, T., Yuan, W., et al. 2006, ApJS, 166, 128 